\title{
Critical Casimir effect for colloids close to chemically patterned substrates
}

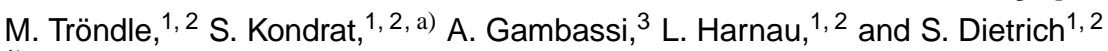 \\ ${ }^{1)}$ Max-Planck-Institut für Metallforschung, Heisenbergstr. 3, D-70569 Stuttgart, Germany \\ ${ }^{2)}$ Institut für Theoretische und Angewandte Physik, Universität Stuttgart, Pfaffenwaldring 57, D-70569 Stuttgart, \\ Germany \\ ${ }^{3)}$ SISSA - International School for Advanced Studies and INFN, via Bonomea 265, 34136 Trieste, \\ Italy \\ (Dated: May, 6 2010)
}

Colloids immersed in a critical or near-critical binary liquid mixture and close to a chemically patterned substrate are subject to normal and lateral critical Casimir forces of dominating strength. For a single colloid we calculate these attractive or repulsive forces and the corresponding critical Casimir potentials within mean-field theory. Within this approach we also discuss the quality of the Derjaguin approximation and apply it to Monte Carlo simulation data available for the system under study. We find that the range of validity of the Derjaguin approximation is rather large and that it fails only for surface structures which are very small compared to the geometric mean of the size of the colloid and its distance from the substrate. For certain chemical structures of the substrate the critical Casimir force acting on the colloid can change sign as a function of the distance between the particle and the substrate; this provides a mechanism for stable levitation at a certain distance which can be strongly tuned by temperature, i.e., with a sensitivity of more than $200 \mathrm{~nm} / \mathrm{K}$.

PACS numbers: 05.70.Jk, 82.70.Dd, 68.35.Rh

\section{INTRODUCTION}

Since the discovery of the Casimir effect in quantum electrodynamics ${ }^{1,2}$ it is well-known that the inherent fluctuations of a medium lead to an effective force acting on its confining boundaries. In soft matter physics, the analogue of the vacuum fluctuations in quantum electrodynamics are the thermal fluctuations of the order parameter $\phi$ of a fluid. These occur on the length scale of the bulk correlation length $\xi$ which is generically of molecular size. However, upon approaching a critical point at the temperature $T=T_{c}$, the correlation length $\xi$ increases with an algebraic singularity and attains macroscopic values. The confinement of these long-ranged fluctuations results in the so-called critical Casimir force acting on a length scale set by $\xi^{3}$. Since the correlation length diverges as $\xi\left(T \rightarrow T_{c}\right) \propto\left|T-T_{c}\right|^{-v}$, where $v$ is a standard bulk critical exponent, the range of the critical Casimir force (and therefore its strength at a certain distance) can be controlled and tuned by minute temperature changes (see, e.g., Refs. 4 and 5). The characteristic energy scale of the critical Casimir effect is given by $k_{B} T_{c}$, which allows for a direct measurement of the critical Casimir forces, in particular if the critical point is located at ambient thermodynamic conditions 6,7 .

The attractive or repulsive character of the critical Casimir force can be controlled by suitable treatments of the confining surfaces. Generically, the surfaces which confine a binary liquid mixture preferentially adsorb one of its two components (or the gas or liquid phase in the case of a one-component fluid). This can be described by effective, symmetry breaking surface fields, which lead to a preference for either positive $[(+)]$ or negative $[(-)]$ values of the scalar order parameter $\phi$, corresponding to the difference between the local concentrations of the two species (or the deviation of the density of the one-component fluid from its critical value). The critical Casimir force strongly depends on the effective boundary

\footnotetext{
a) Present address: Department of Chemistry, Imperial College London, South Kensington Campus, SW7 2AZ London, U.K.
}

conditions (BC) at the walls (see, e.g., Refs. $8-15$ and references therein). It is attractive for equal symmetry breaking $( \pm, \pm) \mathrm{BC}$ and repulsive for opposing $( \pm, \mp) \mathrm{BC}$. Inter alia, this latter feature qualifies critical Casimir forces to be a tool to overcome the problem of "stiction" which occurs in microand nano-mechanical devices. (The quantum electrodynamic Casimir force is typically attractive and thus responsible for stiction; turning it to be repulsive requires a careful choice of the fluid and of the bulk materials of the confinement ${ }^{16}$.) The theoretical description of the critical Casimir forces is particularly challenging due to the non-Gaussian character of the order parameter fluctuations, which contrasts with the intrinsically Gaussian nature of the low energy fluctuations of the electromagnetic field; in addition, the critical Casimir effect is also particularly rich as it allows, inter alia, symmetry breaking boundary conditions, which do not occur for electromagnetic fields.

The critical Casimir effect exhibits universality, i.e., the critical Casimir force expressed in terms of suitable scaling variables depends only on the universality class of the bulk critical point and on the type of boundary conditions, whereas it is independent of the microscopic structure and of the material properties of the specific fluid medium involved. In our present theoretical analysis we focus on the Ising universality class which encompasses the experimentally relevant classical binary liquid mixtures and simple fluids.

The existence of the critical Casimir effect has been experimentally confirmed and its strength has been first measured indirectly for wetting films ${ }^{17-20}$. The first direct measurement of this effect has been performed at the sub-micrometer scale for a spherical colloid immersed in a (near) critical binary liquid mixture close to a laterally homogeneous and planar substrate ${ }^{6,7}$. The corresponding Monte Carlo simulation data for the film geometry are in very good quantitative agreement with all available experimental data ${ }^{6,7,21}-24$. Theoretical studies of the critical Casimir effect acting on colloidal particles involve spherically ${ }^{25-29}$ or ellipsoidally ${ }^{30}$ shaped colloids adjacent to homogeneous substrates.

Besides their wide use as model systems in soft matter 


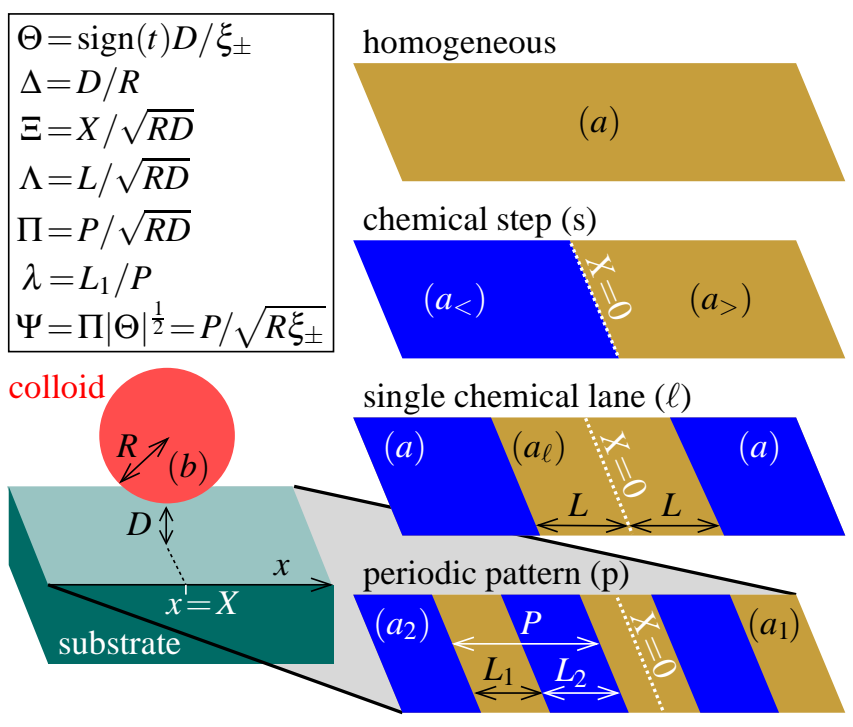

FIG. 1. Sketch of a spherical colloid immersed in a near-critical binary liquid mixture (not shown) and close to a (patterned) planar substrate. The sphere with $(b)$ boundary condition (BC) and radius $R$ is located at a surface-to-surface distance $D$ from the substrate and its center has a lateral coordinate $x=X$ with the substrate pattern being translationally invariant in all other directions. The following four different types of substrate surfaces are considered: homogeneous substrate [Sec. III], a chemical step [s; Sec. IV], a single chemical lane $[\ell ; \operatorname{Sec} . \nabla]$, and a periodically patterned substrate $[\mathrm{p} ; \operatorname{Sec} . \mathrm{VI}]$. (Note that for a four-dimensional system, which we also consider, this is a three-dimensional cut of the system, which is invariant along the fourth direction; the sphere thus corresponds to a hypercylinder in four dimensions.) For later reference, the box on the left side summarizes the definitions of the various scaling variables which the scaling functions of the critical Casimir force depend on for the listed geometrical configurations. On the right, $(a),\left(a_{\gtrless}\right),\left(a_{\ell}\right),\left(a_{1}\right)$, and $\left(a_{2}\right)$ indicate the boundary conditions corresponding to the various chemical patterns.

physics, colloids have applications at the micro- and nanometer scale. In this context, they are widely used in micro- and nano-mechanical devices. Therefore, one may utilize the critical Casimir forces acting on colloids because their strength and their direction can be tuned in a controlled way. Suitably designed chemically or geometrically structured substrates generate lateral critical Casimir forces acting on colloidal particles ${ }^{31-34}$. Current techniques allow one to endow solid surfaces with precise structures on the nano- and micrometerscale. Hence, the critical Casimir effect can be used to create laterally confining potentials for a single colloid, which can be tuned by temperature ${ }^{32}$.

Recently, the critical Casimir potential of a colloid close to a substrate with a pattern of parallel chemical stripes with laterally alternating adsorption preference has been measured 32 . In our corresponding theoretical study $\stackrel{31}{ }$, we have calculated the normal and lateral critical Casimir forces acting on a colloid close to such a patterned substrate as well as the corresponding potentials. We have used our theoretical predictions for the universal scaling functions of the critical Casimir potential in order to interpret the available experimental data in Ref. 32. It has turned out that an agreement between theory and experiment can be achieved only if one takes into account the geometrical details of the chemical substrate pattern. This demonstrates that the critical Casimir effect is very sensitive to the details of the imprinted structures and that it can resolve them.

Here we generalize our previous analysis ${ }^{31}$ to various substrate patterns. In particular we study the critical Casimir effect for a three-dimensional sphere close to a homogeneous substrate [Sec. [II]], a chemical step [Sec. IV], a single chemical lane $[\mathrm{Sec} . \bar{\nabla}]$, and periodic patterns of chemical stripes of alternating adsorption preference [Sec. VI] [see Fig. 1]. For completeness, we also consider a cylinder which is aligned with the chemical pattern [Sec. [VII]. We provide quantitative predictions for the scaling functions of the critical Casimir forces, pursuing a two-pronged approach: (i) We calculate the force using the full three-dimensional numerical analysis of the appropriate mean-field theory (MFT). (ii) We use the so-called Derjaguin approximation (DA) based on the scaling functions for the critical Casimir force in the film geometry either obtained analytically within MFT 35 or obtained

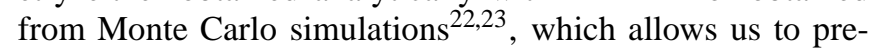
dict the critical Casimir force in the physically relevant threedimensional case. Inter alia, we determine the range of validity of the DA within MFT, which provides guidance concerning its applicability in three spatial dimensions $d=3$. This is an important information because presently available Monte Carlo simulations are far from being able to capture complex geometries 22,23 .

Currently, the possibility of realizing stable levitation of particles by means of the electrodynamic Casimir forces has been the subject of intense theoretical investigation ${ }^{36-41}$. Our results presented in Secs. VI and VII show that for suitable choices of the geometry of the chemical pattern of the substrate, the critical Casimir forces can be used to levitate a colloid above the substrate at a height which can be tuned by temperature. This levitation is stable against perturbations because it corresponds to a minimum of the potential of the critical Casimir force acting on the colloid.

In Sec. [I] we briefly introduce the necessary terminology related to finite-size scaling and we discuss briefly the corresponding MFT. Section [II is devoted to the well-studied case of a colloid close to a homogeneous substrate. (In $d=4$, as appropriate for MFT, the three-dimensional colloid is extended to the fourth dimension as a hypercylinder, for which we also present the results of our analysis.) As mentioned above, the various patterns and setups are considered in Secs. IV VII] We conclude and summarize our findings in Sec. VIII. Certain important technical details concerning the calculation of the Derjaguin approximation are presented in the Appendices A-D

\section{THEORETICAL BACKGROUND}

\section{A. Finite-size scaling}

According to the theory of finite-size scaling, the normal and lateral critical Casimir forces and the corresponding potentials can be described by universal scaling functions, which are independent of the molecular details of the system but depend only on the gross features of the system, i.e., on the bulk universality class (see, e.g., Refs. 8 and 9 and references therein) of the associated critical point. Here, we focus on the 
Ising universality class (which is characterized by a scalar order parameter $\phi$ ) in spatial dimensions $d=3$ and $d=4$. In addition, the critical Casimir force depends on the type of effective boundary conditions at the walls, which we denote by $(a)$ and $(b)$, and by the geometry of the confining surfaces $42-44$. Note that $(a)$ and $(b)$ can represent the various symmetry preserving fixed-point BC (the so-called ordinary, special, periodic, or antiperiodic boundary conditions ${ }^{8.9}$ ) in addition to the symmetry breaking cases $( \pm)$ we are mainly interested in, and which describe the adsorption of fluids at the confining walls.

Inspired by the experiments described in Ref. 32 we consider binary liquid mixtures with their consolute critical point approached by varying the temperature $T$ towards $T_{c}$ at fixed pressure and critical composition. We first study the film geometry in which the fluid undergoing the continuous phase transition is confined between two parallel, infinitely extended walls at distance $L$. According to renormalization group theory the normal critical Casimir force $f_{(a, b)}$ per unit area which is acting on the walls scales as $\frac{10}{}$

$$
f_{(a, b)}(L, T)=k_{B} T \frac{1}{L^{d}} k_{(a, b)}\left(\operatorname{sign}(t) L / \xi_{ \pm}\right),
$$

where $(a, b)$ denotes the pair of boundary conditions $(a)$ and (b) characterizing the two walls. The scaling function $k_{(a, b)}$ depends only on a single scaling variable given by the sign of the reduced temperature distance $t$ from the critical point ( \pm for $t \gtrless 0$ ) and the film thickness $L$ in units of the bulk correlation length $\xi_{ \pm}\left(t \rightarrow 0^{ \pm}\right)=\xi_{0}^{ \pm}|t|^{-v}$, where $v \simeq 0.63$ in $d=3$ and $v=1 / 2$ in $d=4^{45}$. (Clearly, one has $f_{(a, b)}(L, T)=$ $f_{(b, a)}(L, T)$.) Positive values of $t, t>0$, correspond to the disordered (homogeneous) phase of the fluid, whereas negative values of $t, t<0$, correspond to the ordered (inhomogeneous) phase, where phase separation occurs. Typically, the homogeneous phase is found at high temperatures, and one has $t=\left(T-T_{c}\right) / T_{c}$. However, many experimentally relevant binary liquid mixtures exhibit a lower critical point, for which the homogeneous phase corresponds to the lowtemperature phase and one has $t=-\left(T-T_{c}\right) / T_{c} c^{6.7}$. The two non-universal amplitudes $\xi_{0}^{ \pm}$of the correlation length are of molecular size and characterized by the universal ratio $\xi_{0}^{+} / \xi_{0}^{-} \simeq 1.9$ in $d=3^{45.46}$ and $\xi_{0}^{+} / \xi_{0}^{-}=\sqrt{2}$ in $d=4^{47} ; \xi_{ \pm}$is determined by the exponential spatial decay of the two-point correlation function of the order parameter $\phi$ in the bulk.

At the critical point $T=T_{c}$, the correlation length diverges, $\xi_{ \pm} \rightarrow \infty$, and the scaling function of the critical Casimir force acting on the two planar walls attains a universal constant value referred to as the critical Casimir amplitude ${ }^{8.9}$ :

$$
k_{(a, b)}\left(L / \xi_{ \pm}=0\right)=\Delta_{(a, b)} .
$$

Away from criticality, the critical Casimir force decays exponentially as a function of $L / \xi_{ \pm}$. For the specific case of symmetry breaking $\mathrm{BC} a, b \in\{+,-\}$ and for $t>0$ one expects for $L / \xi_{+} \gg 1$ a pure exponential decay of $f_{(++)}$(see, e.g., Refs. 11, 35, and 48 and footnote 3 in Ref. 31, i.e., a decay without an algebraic prefactor to the exponential and without a numerical prefactor to $L / \xi_{+}$in the argument of the exponential) corresponding to

$$
k_{(+, \pm)}\left(L / \xi_{+} \gg 1\right)=A_{ \pm}\left(\frac{L}{\xi_{+}}\right)^{d} \exp \left(-L / \xi_{+}\right),
$$

where $A_{ \pm}$are universal constants ${ }^{7}$. Note that, in the absence of symmetry-breaking fields inside the film, the scaling functions for $(+,+) \mathrm{BC}$ are the same as for $(-,-) \mathrm{BC}$.

\section{B. Mean-field theory}

The standard Landau-Ginzburg-Wilson fixed-point effective Hamiltonian describing critical phenomena of the Ising universality class is given by 42,43

$$
\mathscr{H}[\phi]=\int_{V} \mathrm{~d}^{d} \mathbf{r}\left\{\frac{1}{2}(\nabla \phi)^{2}+\frac{\tau}{2} \phi^{2}+\frac{u}{4 !} \phi^{4}\right\},
$$

where $\phi(\mathbf{r})$ is the order parameter describing the fluid, which completely fills the volume $V$ in $d$-dimensional space. The first term in the integral in Eq. (4) penalizes local fluctuations of the order parameter. The parameter $\tau$ in Eq. (4) is proportional to $t$, and the coupling constant $u$ is positive and provides stability of the Hamiltonian for $t<0$. The mean-field order parameter profile $m:=u^{1 / 2}\langle\phi\rangle$ minimizes the Hamiltonian, i.e., $\delta \mathscr{H}[\phi] /\left.\delta \phi\right|_{\phi=u^{-1 / 2} m}=0$. In the bulk the mean-field order parameter is spatially constant and attains the values $\langle\phi\rangle=$ $\pm a|t|^{\beta}$ for $t<0$ and $\langle\phi\rangle=0$ for $t>0$, where, besides $\xi_{0}^{+}, a$ is the only additional independent non-universal amplitude appearing in the description of bulk critical phenomena ${ }^{42,43}$, and $\beta(d=4)=1 / 2$ is a standard critical exponent. Within MFT $\tau=t\left(\xi_{0}^{+}\right)^{-2}$ and $u=6 a^{2}\left(\xi_{0}^{+}\right)^{-2}$. In a finite-size system the bulk Hamiltonian $\mathscr{H}[\phi]$ is supplemented by appropriate surface and curvature (edge) contributions ${ }^{42.43}$. In the strong adsorption limit ${ }^{49.50}$, these contributions generate boundary conditions for the order parameter such that $\left.\phi\right|_{\text {surface }}= \pm \infty$. For binary liquid mixtures these fixed-point $( \pm) \mathrm{BC}$ are the experimentally relevant ones. (Note that a weak adsorption preference might lead to a crossover between various kinds of effective boundary conditions for the order parameter $\phi^{7.14 .15}$.)

We have minimized numerically $\mathscr{H}[\phi]$ using a $3 d$ finite element method in order to obtain the (spatially inhomogeneous) profile $m(\mathbf{r})$ for the geometries under consideration [see Fig. 1]. The normal and the lateral critical Casimir forces are calculated directly from these mean-field order parameter profiles using the stress tensor ${ }^{30,35}$. This allows one to infer the universal scaling functions of the critical Casimir forces at the upper critical dimension $d=4$ up to an overall prefactor $\propto u^{-1}$ and up to logarithmic corrections. The corresponding critical Casimir potential is obtained by the appropriate integration of the normal or of the lateral critical Casimir forces.

In the case of planar walls the MFT scaling functions for the critical Casimir force can be determined analytically 35 and one finds [see Eq. (2)] for the case of symmetry breaking boundary conditions the following critical Casimir amplitudes: $\Delta_{(+,+)}=\Delta_{(-,-)}=24[K(1 / \sqrt{2})]^{4} / u \simeq-283.61 \times u^{-1}$, where $K$ is the complete elliptic integral of the first kind, and $\Delta_{(+,-)}=-4 \Delta_{(+,+)}$[see Ref. 35 and Eq. (27) and Ref. [49] in Ref. 23].

In $d=4$ (corresponding to MFT) the three-dimensional sphere is a hypercylinder and the physical properties are invariant along the fourth dimension. Accordingly, the MFT results for the force and the potential given below are those per length along this additional direction. 


\section{HOMOGENEOUS SUBSTRATE}

We first consider a three-dimensional sphere of radius $R$ with $(b) \mathrm{BC}$ facing a chemically homogeneous substrate with (a) BC at a surface-to-surface distance $D$ as shown in Fig. 1 , denoting this combination by $(a, b)$. The critical Casimir force $F_{(a, b)}(D, R, T)$ normal to the substrate surface and the corresponding critical Casimir potential $\Phi_{(a, b)}(D, R, T)=$ $\int_{D}^{\infty} \mathrm{d} z F_{(a, b)}(z, R, T)$ take the scaling forms $6,7,27,31$

$$
F_{(a, b)}(D, R, T)=k_{B} T \frac{R}{D^{d-1}} K_{(a, b)}(\Theta, \Delta)
$$

and

$$
\Phi_{(a, b)}(D, R, T)=k_{B} T \frac{R}{D^{d-2}} \vartheta_{(a, b)}(\Theta, \Delta)
$$

where $\Delta=D / R$ and $\Theta=\operatorname{sign}(t) D / \xi_{ \pm}$(for $t \gtrless 0$ ) are the scaling variables corresponding to the distance $D$ in units of the radius $R$ of the colloid and of the correlation length $\xi_{ \pm}$, respectively. The case $d=4$ corresponds to the MFT solution up to logarithmic corrections, which we shall neglect here. Equations (5) and (6) describe a force and an energy, respectively, per $D^{d-3}$, which for $d=4$ corresponds to considering $F_{(a, b)}$ and $\Phi_{(a, b)}$ per length $L_{4}$ of the extra translationally invariant direction of the hypercylinder.

\section{A. Derjaguin approximation}

The Derjaguin approximation (DA) is based on the idea of decomposing the surface of the spherical colloid into infinitely thin circular rings of radius $\rho$ and area $\mathrm{d} S(\rho)=2 \pi \rho \mathrm{d} \rho$ which are parallel to the opposing substrate surface $6,7,27,31,51$. (Here we do not multiply $2 \pi \rho \mathrm{d} \rho$ by the linear extension $L_{4}$ of the hypercylinder along its axis in the fourth dimension, because the critical Casimir force is eventually expressed in units of $L_{4}$, which therefore drops out from the final expressions.) The distance $L$ of a ring with radius $\rho$ from the substrate is given by

$$
L(\rho)=D+R\left(1-\sqrt{1-\rho^{2} / R^{2}}\right)
$$

Assuming additivity of the forces and neglecting edge effects, the normal critical Casimir forces $\mathrm{d} F(\rho)$ acting on these rings can be expressed in terms of the force acting on parallel plates [Eq. (1)]:

$$
\frac{\mathrm{d} F(\rho)}{k_{B} T}=\frac{\mathrm{d} S}{[L(\rho)]^{d}} k_{(a, b)}\left(\operatorname{sign}(t) L(\rho) / \xi_{ \pm}\right) .
$$

Finally, in order to calculate the total force $F_{(a, b)}$ acting on the colloid, one sums up the contributions of the rings, which yields

$$
\frac{F_{(a, b)}(D, R, T)}{k_{B} T} \simeq 2 \pi \int_{0}^{R} \mathrm{~d} \rho \rho[L(\rho)]^{-d} k_{(a, b)}\left(\operatorname{sign}(t) L(\rho) / \xi_{ \pm}\right) .
$$

(For $d=3, F_{(a, b)}$ is the force on a sphere whereas in $d=4$ it is the force on a hypercylinder per length of its axis.)
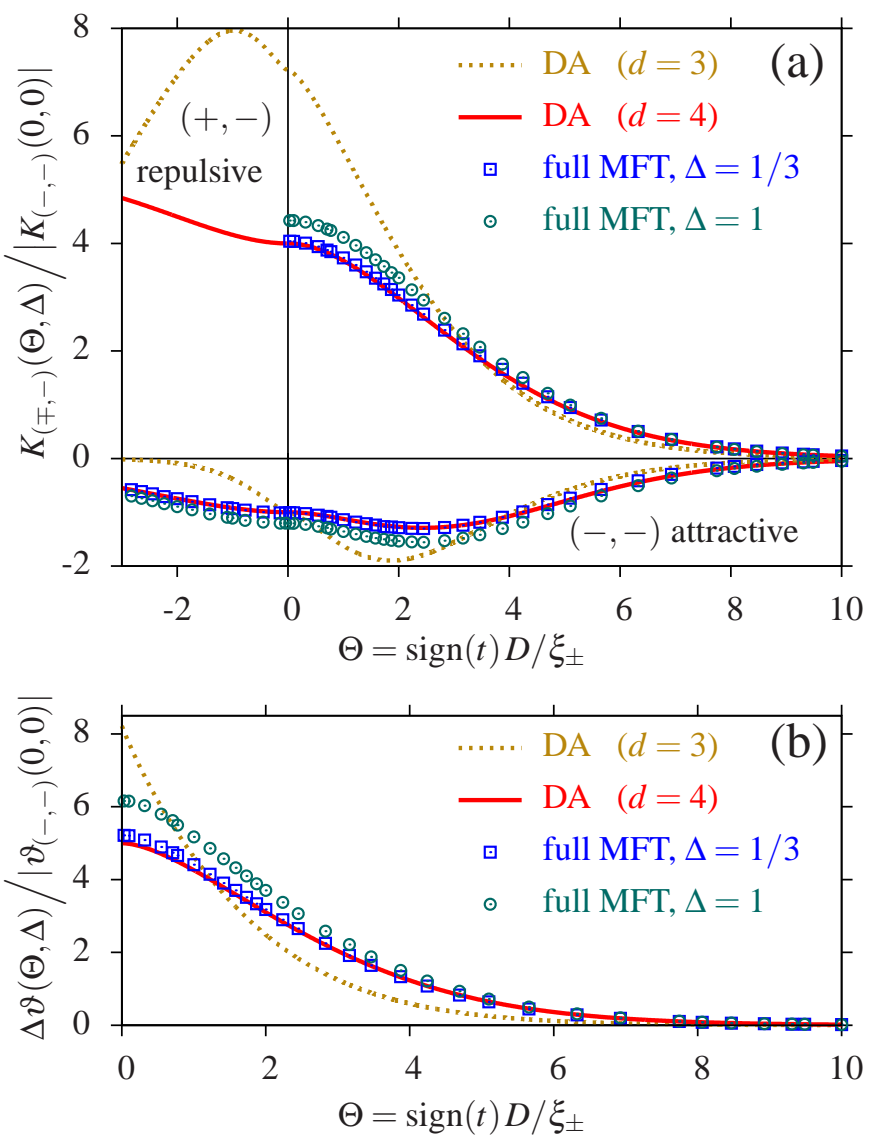

FIG. 2. (a) Scaling functions $K_{(\mp,-)}$ for the normal critical Casimir force [Eq. [5] acting on a three-dimensional sphere with $(b)=(-)$ BC close to a homogeneous substrate with $(a)=(\mp)$ BC [Fig. 1]. The suitably normalized scaling functions $K_{(\mp,-)}$ are shown as a function of the scaling variable $\Theta=\operatorname{sign}(t) D / \xi_{ \pm}$for $t \gtrless 0$, where $t$ is the reduced deviation from the critical temperature and $K_{(-,-)}(0,0)$ is the value of the critical Casimir force scaling function within the DA at $T=T_{c}$ for $(-,-)$ BC. The solid lines correspond to the Derjaguin approximation (DA, $\Delta=D / R \rightarrow 0$ ) within mean-field theory (MFT, $d=4$ ) whereas the dotted lines correspond to the DA obtained by using Monte Carlo (MC) results for films in $d=3$ the systematic uncertainties of which are not indicated ${ }^{52}$. The normalization implies that at $\Theta=0$ both the solid and dotted lines pass through -1 for $(-,-)$ BC whereas the solid line passes through 4 for $(+,-) \mathrm{BC}$. The symbols correspond to the full numerical MFT results obtained for $\Delta=1 / 3$ and $\Delta=1$, the size of which indicates the estimated numerical error. (For $(+,-) \mathrm{BC}$ and $t<0$ we have not been able to calculate the corresponding scaling functions with adequate precision due to severe numerical difficulties in obtaining the full threedimensional order parameter profile in the presence of two "competing" bulk values.) Since within the DA the dependence of $K_{(\mp,-)}$ on $\Delta$ drops out, the difference between the symbols $\square$ and $\odot$ and the solid lines measures the accuracy of the DA in $d=4$. (b) Difference $\Delta \vartheta=\vartheta_{(+,-)}-\vartheta_{(-,-)}$of the scaling functions for the Casimir potentials [Eq. [6] for $(+,-)$ and $(-,-)$ BC, suitably normalized by $\vartheta_{(-,-)}(0,0)$. The solid line corresponds to the DA within MFT and the symbols correspond to the full MFT results for $\Delta=1 / 3$ and $\Delta=1$; the dotted line is the DA for $d=3$. Due to the normalization the solid line reaches 5 for $\Theta=0$. 
One expects the DA to describe the actual behavior accurately if the colloid is very close to the substrate, i.e., for $\Delta=D / R \rightarrow 0$. In this limit, Eq. (7) can be approximated by $L(\rho)=D \alpha$ where $\alpha=1+\rho^{2} /(2 R D)$, so that one finds for the scaling function of the force ${ }^{7,27}$

$$
K_{(a, b)}(\Theta, \Delta \rightarrow 0)=2 \pi \int_{1}^{\infty} \mathrm{d} \alpha \alpha^{-d} k_{(a, b)}(\alpha \Theta)
$$

and, accordingly, for the scaling function of the potential ${ }^{6,7,31}$

$$
\vartheta_{(a, b)}(\Theta, \Delta \rightarrow 0)=2 \pi \int_{1}^{\infty} \mathrm{d} \beta\left(\frac{1}{\beta^{d-1}}-\frac{1}{\beta^{d}}\right) k_{(a, b)}(\beta \Theta)
$$

At the bulk critical point, using Eq. (2), one finds the well known values $K_{(a, b)}(0,0)=2 \pi \Delta_{(a, b)} /(d-1)$ and $\vartheta_{(a, b)}(0,0)=2 \pi \Delta_{(a, b)} /[(d-2)(d-1)]$. We note that the DA implies that the dependence of $F_{(a, b)}$ and $\Phi_{(a, b)}$ on the size $R$ of the sphere reduces to the proportionality $\propto R$ indicated explicitly in Eqs. (5) and (6).

Before proceeding further one first has to assess the accuracy of the DA, which will carried out below within MFT $(d=4)$. We expect the range of validity of the DA to be similar for $d=3$, so that within that range one can use the DA based on scaling functions for the film geometry obtained from Monte Carlo simulations 52 in order to calculate the critical Casimir force acting on a colloid in $d=3$.

\section{B. Scaling functions for the normal critical Casimir force and the potential}

The expressions obtained above within the DA hold for general boundary conditions $(a)$ and $(b)$ and are valid beyond the cases we consider in the following, i.e., $a \in\{+,-\}$ and $b=-$. Figure 2 (a) shows the full numerical MFT $(d=4)$ results for the scaling functions $K_{( \pm,-)}$with $\Delta=1$ and $\frac{1}{3}$ compared with the corresponding DA results based on the suitable numerical integration [Eq. [10]] of the analytic (MFT) expression for $k_{( \pm,-)}{ }^{35}$. Moreover, in Fig. 2, the corresponding DA results for $d=3$ are shown; they are obtained from the film scaling functions determined by MC simulations 52 and by using the corresponding ratio of the correlation lengths above and below $T_{c}{ }^{45}$. In Fig. 2 (b) we report the difference $\Delta \vartheta(\Theta, \Delta):=\vartheta_{(+,-)}(\Theta, \Delta)-\vartheta_{(-,-)}(\Theta, \Delta)$ computed for the various cases reported in Fig. 22a), which will be useful for describing the case of a chemically patterned substrate. The scaling functions in $d=4$ are reasonably well reproduced by the DA for $\Delta \lesssim 0.4$ and we expect this to hold for $d=3$ as well. The fact that for increasing values of $\Delta$ the magnitude of the actual scaling functions becomes larger compared with those within the DA (corresponding to $\Delta \rightarrow 0$ ) is in agreement with earlier results obtained for a $d$-dimensional hypersphere (see, e.g., Ref.27).

It has been shown that the scaling functions obtained within the DA for $d=3$ agree very well - within the experimental accuracy - with the ones obtained from direct measurements of the critical Casimir potential ${ }^{6,7}$ corresponding to $\Delta \lesssim 0.35$ (see also Ref. [48] in Ref. 23).

\section{CHEMICAL STEP (s)}

The basic building block of a chemically patterned substrate of the type we consider here, i.e., with translational invariance in all directions but one $(x)$, is a chemical step (s) realized by a substrate with $\left(a_{\gtrless}\right)$ BC for $x \gtrless 0$ at its surface. In this section we analyze the critical Casimir force if such a substrate is approached by a colloid with $(b)$ BC with its center located at the lateral position $x=X$ (see Fig. 1 and Ref. 32 for experimental realizations). We denote this configuration by $\left(a_{<} \mid a_{>}, b\right)$. The normal critical Casimir force $F_{\mathrm{s}}$ is described by the scaling form ${ }^{31}$

$$
F_{\mathrm{S}}(X, D, R, T)=k_{B} T \frac{R}{D^{d-1}} K_{\mathrm{S}}(\Xi, \Theta, \Delta),
$$

where $\Xi=X / \sqrt{R D}$ is the scaling variable corresponding to the lateral position of the colloid. It is useful to write the scaling function $K_{\mathrm{s}}$ as

$$
\begin{aligned}
K_{\mathrm{S}}(\Xi, \Theta, \Delta)= & \frac{K_{\left(a_{<}, b\right)}+K_{\left(a_{>}, b\right)}}{2} \\
& +\frac{K_{\left(a_{<}, b\right)}-K_{\left(a_{>}, b\right)}}{2} \psi_{\left(a_{<} \mid a_{>}, b\right)}(\Xi, \Theta, \Delta),
\end{aligned}
$$

where the scaling functions of the laterally homogeneous substrates $K_{\left(a_{\gtrless}, b\right)}$ depend on $\Theta$ and $\Delta$ only [Eq. (5)], and the scaling function $\psi_{\left(a_{<} \mid a_{>}, b\right)}$ varies from +1 at $\Xi \rightarrow-\infty$ to -1 at $\Xi \rightarrow+\infty$, such that the laterally homogeneous cases are recovered far from the step. Accordingly, the corresponding critical Casimir potential $\Phi_{\mathrm{s}}(X, D, R, T)=\int_{D}^{\infty} \mathrm{d} z F_{\mathrm{s}}(X, z, R, T)$ can be cast in the form 31

$$
\Phi_{\mathrm{S}}(X, D, R, T)=k_{B} T \frac{R}{D^{d-2}} \vartheta_{\mathrm{s}}(\Xi, \Theta, \Delta),
$$

and

$$
\begin{aligned}
\vartheta_{\mathrm{S}}(\Xi, \Theta, \Delta)= & \frac{\vartheta_{\left(a_{<}, b\right)}+\vartheta_{\left(a_{>}, b\right)}}{2} \\
& +\frac{\vartheta_{\left(a_{<}, b\right)}-\vartheta_{\left(a_{>}, b\right)}}{2} \omega_{\left(a_{<} \mid a_{>}, b\right)}(\Xi, \Theta, \Delta),
\end{aligned}
$$

where $\vartheta_{\left(a_{\gtrless}, b\right)}$ depend on $\Theta$ and $\Delta$ only [Eq. [6], and $\omega_{\left(a_{<} \mid a_{>}, b\right)}(\Xi= \pm \infty, \Theta, \Delta)=\mp 1$. Note that the scaling functions $\psi_{\left(a_{<} \mid a_{>}, b\right)}$ and $\omega_{\left(a_{<} \mid a_{>}, b\right)}$ are independent of the common prefactor $\propto u^{-1}$ [see Sec. IIB], which is left undetermined by the analytical and numerical mean-field calculation of $K_{\mathrm{s}}$ and $\vartheta_{\text {s. }}$.

\section{A. Derjaguin approximation}

If the sphere is close to the substrate, i.e., $\Delta \rightarrow 0$, the DA can be applied, and one finds for the scaling function of the critical Casimir force [see Appendix A]

$$
\begin{aligned}
& \psi_{\left(a_{<} \mid a_{>}, b\right)}(\Xi \gtrless 0, \Theta, \Delta \rightarrow 0)=\mp 1 \\
& \pm \frac{4 \int_{1+\Xi^{2} / 2}^{\infty} \mathrm{d} \alpha \alpha^{-d} \arccos \left(|\Xi|(2 \alpha-2)^{-1 / 2}\right) \Delta k(\alpha \Theta)}{K_{\left(a_{<}, b\right)}(\Theta, \Delta \rightarrow 0)-K_{\left(a_{>}, b\right)}(\Theta, \Delta \rightarrow 0)}
\end{aligned}
$$


where $\Delta k(\Theta)=k_{\left(a_{<}, b\right)}(\Theta)-k_{\left(a_{>}, b\right)}(\Theta)$ is the difference between the scaling functions for the critical Casimir forces acting on two planar walls with $\left(a_{<}, b\right)$ and with $\left(a_{>}, b\right)$ boundary conditions, respectively. We note that according to Eqs. (16) and (10) within the DA $\psi_{\left(a_{<} \mid a_{>}, b\right)}$ can be determined from the knowledge of the film scaling functions $k_{(a, b)}(\Theta)$ [Eq. (1)] only. Due to the assumption of additivity which underlies the DA, (i) $\psi_{\left(a_{<} \mid a_{>}, b\right)}$ vanishes at $\Xi=0$ for all $\Theta$ and it is an antisymmetric function of $\Xi$ and (ii) $\psi_{\left(a_{<} \mid a_{>}, b\right)}=\psi_{\left(a_{>} \mid a_{<}, b\right)}$; within the DA both of these properties are valid irrespective of the type of boundary conditions on both sides of the chemical step. (However, the actual scaling function $\psi_{\left(a_{<} \mid a_{>}, b\right)}$ as, e.g., obtained from full numerical MFT calculations may violate this symmetry because the actual critical Casimir forces are non-additive.) At the bulk critical point one has $\Theta=0$ so that [see Appendix A1],

$$
\begin{aligned}
& \psi_{\left(a_{<} \mid a_{>}, b\right)}(\Xi, \Theta=0, \Delta \rightarrow 0)= \\
& \Xi^{2 d-7}\left(\frac{15}{2}(3-d)+(3-2 d) \Xi^{2}-\Xi^{4}\right)\left(2+\Xi^{2}\right)^{-\left(d-\frac{3}{2}\right)}
\end{aligned}
$$

independent of $k_{\left(a_{\gtrless}, b\right)}$. Similarly, within the DA one finds for the scaling function $\omega$ of the critical Casimir potential [see Appendix A and Ref. 31]

$$
\begin{aligned}
& \omega_{\left(a_{<} \mid a_{>}, b\right)}(\Xi \gtrless 0, \Theta, \Delta \rightarrow 0)=\mp 1 \\
& \quad \pm \frac{\Xi^{4} \int_{1}^{\infty} \mathrm{d} s \frac{\operatorname{sarccos}\left(s^{-1 / 2}\right)-\sqrt{s-1}}{\left(1+\Xi^{2} s / 2\right)^{d}} \Delta k\left(\Theta\left[1+\Xi^{2} s / 2\right]\right)}{\vartheta_{\left(a_{<}, b\right)}(\Theta, \Delta \rightarrow 0)-\vartheta_{(a>b)}(\Theta, \Delta \rightarrow 0)} .
\end{aligned}
$$

This yields $\omega_{\left(a_{<} \mid a_{>}, b\right)}(\Xi=0, \Theta, \Delta \rightarrow 0)=0$, as expected from the underlying assumption of additivity; within full MFT this only holds in the limit $\Delta \rightarrow 0$. At the critical point we find [see Appendix A1

$$
\omega_{\left(a_{<} \mid a_{>}, b\right)}(\Xi, \Theta=0, \Delta \rightarrow 0)=\Xi\left(1-d-\Xi^{2}\right)\left(\Xi^{2}+2\right)^{-3 / 2} .
$$

For symmetry breaking $(\mp,-) \mathrm{BC}$ and $\Theta \gg 1$ the critical Casimir force $f_{(\mp,-)}(D, T)$ acting on two planar walls at a distance $D$ decays $\propto \exp (-\Theta)$ [Eqs. (3) and (10], which within the DA leads to the same $d$-independent result for the scaling functions $\psi_{(+\mid-,-)}$and $\omega_{(+\mid-,-)}$[see Appendix A2]:

$$
\begin{aligned}
\psi_{(+\mid-,-)}(\Xi, \Theta \gg 1, \Delta \rightarrow 0) & \\
\omega_{(+\mid-,-)}(\Xi, \Theta \gg 1, \Delta & \rightarrow 0)= \\
& -\operatorname{erf}(\sqrt{\Theta / 2} \Xi),
\end{aligned}
$$

where erf is the error function.

Figure 3 a) compares the scaling function $\omega_{\left(a_{<} \mid a_{>}, b\right)}$ for the critical Casimir potential of a sphere with $(-) \mathrm{BC}$ in front of a $(+\mid-)$ step, as obtained within the DA for $d=4$ [Eq. [18)], with the one obtained numerically within full MFT for $\Delta=$ $1 / 3$. For $\Delta \lesssim 1 / 3$ the DA captures the scaling function very well, in particular for $\Theta \gtrsim 3 \frac{31}{}$. The scaling function $\omega_{\left(a_{<} \mid a_{>}, b\right)}$ obtained within the DA $(d=3)$ on the basis of the Monte Carlo data of Ref. 23, which is also shown in Fig. 3(a), has been used successfully in order to interpret the experimental data of Ref. 32, for which the analysis in terms of separate, independent, and consecutive chemical steps turned out to be
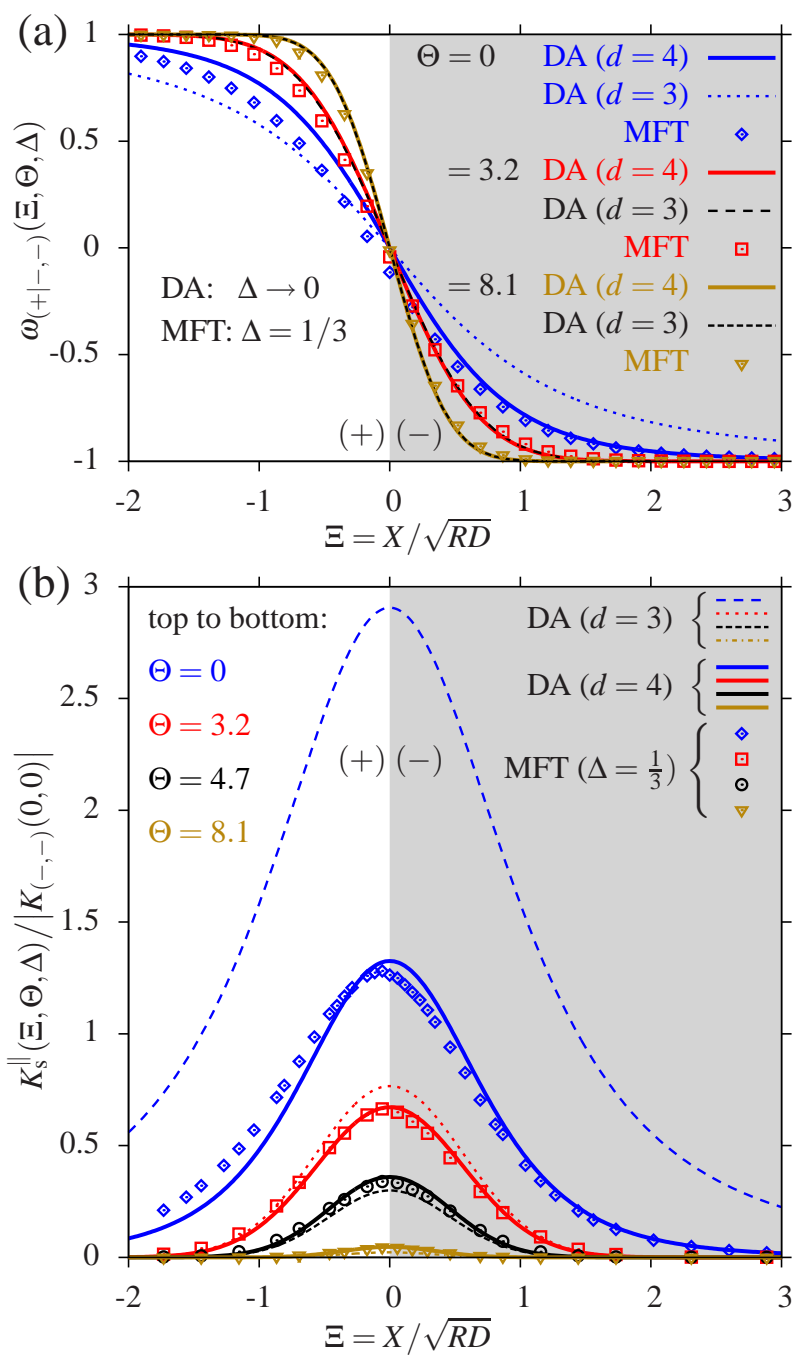

FIG. 3. (a) Scaling function $\omega_{(+\mid-,-)}$[Eq. 15] for the critical Casimir potential of a spherical colloid with (-) BC across a chemical step $(+\mid-)$ as a function of $\Xi \equiv X / \sqrt{R D}$ for various (positive) values of $\Theta=D / \xi_{+}$31. Within the DA $\omega_{(+\mid-,-)}$is an antisymmetric function of $\Xi$ [Eq. (18)] whereas within full MFT this antisymmetry is slightly violated, in particular for small $\Theta$. (b) Corresponding scaling function $K_{\mathrm{s}}^{\|}$[Eq. 21] ] of the lateral critical Casimir force, normalized by the amplitude $K_{(-,-)}(0,0)=2 \pi \Delta_{(-,-)} /(d-1)$ of the normal critical Casimir force at $T=T_{c}$ acting on a colloid with (-) BC close to a homogeneous substrate with (-) BC within the DA [Sec. IIIA]. For both (a) and (b) the full numerical MFT results obtained for $\Delta=1 / 3$ are shown as symbols (the symbol size represents the estimated numerical error) whereas the lines show the corresponding results obtained within the DA (i.e., $\Delta \rightarrow 0$ ); the dotted lines refer to $d=3$ and are obtained by using Monte Carlo simulation data 52 and the solid lines refer to $d=4$. The lines for $\Theta=0$ are obtained by using Eq. (19) and Eq. 23, respectively; for $\Theta=3.2,4.7,8.1$ the DA lines de facto coincide with the asymptotic results obtained for symmetry breaking $\mathrm{BC}$ and $\Theta \gg 1$ [Eq. (20) and Eq. (24), respectively] and thus are indeed independent of $d$. The DA $(d=4)$ provides a good approximation for the full numerical MFT data, in particular for $\Theta \gtrsim 3 . K_{\mathrm{s}}^{\|}>0$ implies that the colloid moves to the right where it enjoys an attractive potential versus a repulsive one for $\Xi<0$. Within the DA $K_{\mathrm{s}}^{\|}$is a symmetric function of $\Xi$ [Eqs. (18) and (22)] whereas within full MFT this symmetry is slightly violated, in particular for small $\Theta$. 
accurate. Moreover, the critical Casimir forces turned out to be a sensitive probe of the chemical pattern and its geometric $\operatorname{design}^{31}$.

\section{B. Lateral critical Casimir force}

The lateral critical Casimir force is given by $F_{\mathrm{s}}^{\|}=-\partial_{X} \Phi_{\mathrm{s}}$ and can be cast in the scaling form

$$
F_{\mathrm{S}}^{\|}(X, D, R, T)=k_{B} T \frac{R}{D^{d-1}}\left(\frac{D}{R}\right)^{1 / 2} K_{\mathrm{s}}^{\|}(\Xi, \Theta, \Delta),
$$

where $K_{\mathrm{s}}^{\|}$is a universal scaling function. $F_{\mathrm{s}}^{\|}$and $K_{\mathrm{s}}^{\|}$vanish far from the chemical step, i.e., for $|\Xi| \rightarrow \infty$. In Eq. (21) the prefactors in terms of $R$ and $D$ and their exponents are chosen such that $K_{\mathrm{s}}^{\|}$is regular and non-vanishing for $\Delta \rightarrow 0$. We note that the same holds for the normal critical Casimir forces and the corresponding potentials [see Eqs. (5), (6), (12), (14), and the considerations following below].

Within the DA $K_{\mathrm{S}}^{\|}$can be calculated from Eqs. 15 and (18):

$$
\begin{aligned}
K_{\mathrm{S}}^{\|}(\Xi, \Theta, \Delta \rightarrow 0)= & \\
-\frac{1}{2}\left[\vartheta_{\left(a_{<, b}\right)}(\Theta, \Delta \rightarrow\right. & \left.0)-\vartheta_{\left(a_{>}, b\right)}(\Theta, \Delta \rightarrow 0)\right] \times \\
& \partial_{\Xi} \omega_{\left(a_{<} \mid a_{>}, b\right)}(\Xi, \Theta, \Delta \rightarrow 0) .
\end{aligned}
$$

At bulk criticality $\Theta=0$ one finds with Eq. (19) [see Eq. (16)]

$$
K_{\mathrm{S}}^{\|}(\Xi, \Theta=0, \Delta \rightarrow 0)=\pi \Delta k(0)\left(2+\Xi^{2}\right)^{-\left(d-\frac{3}{2}\right)} .
$$

For $(\mp,-)$ BC and $\Theta \gg 1$ Eqs. (14), (15), and (20) lead to

$$
\begin{aligned}
& K_{\mathrm{S}}^{\|}(\Xi, \Theta \gg 1, \Delta \rightarrow 0)= \\
& {\left[\vartheta_{(+,-)}(\Theta, \Delta)-\vartheta_{(-,-)}(\Theta, \Delta)\right] \sqrt{\frac{\Theta}{2 \pi}} \exp \left\{-\frac{\Theta \Xi^{2}}{2}\right\},}
\end{aligned}
$$

for both $d=3$ and $d=4$. [The prefactor $\Delta \vartheta(\Theta, \Delta)=$ $\vartheta_{(+,-)}(\Theta, \Delta)-\vartheta_{(-,-)}(\Theta, \Delta)$ in Eq. (24) is shown in Fig. 2(b).]

Figure 3 (b) shows the comparison between the normalized lateral critical Casimir force obtained within the DA (solid lines) and the full MFT data obtained for $\Delta=1 / 3$ (symbols). We infer that not only the shape of $K_{\mathrm{s}}^{\|}$as a function of $\Xi$ but also its amplitude is described well by the DA [Eqs. (23) and [24] ] for $\Delta \lesssim 1 / 3$, and in particular for $\Theta \gtrsim 3$. We expect this feature to hold in $d=3$, too, as well as for the normal critical Casimir force and the critical Casimir potential. The lateral critical Casimir forces for $d=3$ obtained within the DA on the basis of Monte Carlo simulation data for the film geometry 52 are shown in Fig. 3 b) as dashed lines. Compared with the previous curves, these ones have similar shapes but their overall amplitudes in units of the normal critical Casimir force at $\Theta=0$ are significantly different for $\Theta=0$ and $\Theta=3.2$. This difference reflects the analogous one observed in the normalized difference between the corresponding critical Casimir potentials for $(+,-)$ and $(-,-)$ BC, reported in Fig. 2(b).

\section{SINGLE CHEMICAL LANE $(\ell)$}

In this section we consider the case of a colloid with $(b) \mathrm{BC}$ close to a substrate with a single chemical lane $(\ell)$ with $\left(a_{\ell}\right)$ $\mathrm{BC}$ and width $2 L$ in the lateral $x$ direction and which is invariant along the other lateral direction(s). The remaining parts of the substrate are two semi-infinite planes at $|x|>L$ with $(a)$ BC [see Fig.1]. The lateral coordinate $X$ of the center of mass of the sphere along the $x$ direction is chosen to vanish in the center of the chemical lane. One expects that for "broad" lanes a description in terms of two subsequent chemical steps is appropriate [Sec. IV] and Ref. 31], whereas for "narrow" lanes the effects of the two subsequent chemical steps interfere. We find that in addition to the variables characterizing the chemical step [Eq. (12)], a further scaling variable $\Lambda=L / \sqrt{R D}$ emerges naturally, which corresponds to the width of the lane. Accordingly, the normal critical Casimir force $F_{\ell}$ acting on the colloid can be cast in the form

$$
F_{\ell}(L, X, D, R, T)=k_{B} T \frac{R}{D^{d-1}} K_{\ell}(\Lambda, \Xi, \Theta, \Delta),
$$

where $K_{\ell}$ is the corresponding universal scaling function. The critical Casimir potential scales as

$$
\Phi_{\ell}(L, X, D, R, T)=k_{B} T \frac{R}{D^{d-2}} \vartheta_{\ell}(\Lambda, \Xi, \Theta, \Delta),
$$

with $\vartheta_{\ell}$ as the universal scaling function for the potential of a sphere close to a single chemical lane. Analogously to Eqs. (13) and (15) we define $\psi_{\ell}$ and $\omega_{\ell}$ according to

$$
\begin{aligned}
K_{\ell}(\Lambda, \Xi, \Theta, \Delta)= & \frac{K_{(a, b)}+K_{\left(a_{\ell}, b\right)}}{2} \\
& +\frac{K_{(a, b)}-K_{\left(a_{\ell}, b\right)}}{2} \psi_{\ell}(\Lambda, \Xi, \Theta, \Delta),
\end{aligned}
$$

and

$$
\begin{aligned}
& \vartheta_{\ell}(\Lambda, \Xi, \Theta, \Delta)=\frac{\vartheta_{(a, b)}+\vartheta_{\left(a_{\ell}, b\right)}}{2} \\
&+\frac{\vartheta_{(a, b)}-\vartheta_{\left(a_{\ell}, b\right)}}{2} \omega_{\ell}(\Lambda, \Xi, \Theta, \Delta),
\end{aligned}
$$

so that far from the lane $\psi_{\ell}(\Lambda,|\Xi| \gg \Lambda, \Theta, \Delta)=$ $\omega_{\ell}(\Lambda,|\Xi| \gg \Lambda, \Theta, \Delta)=1$. On the other hand, only for a "broad" lane the scaling functions at the center of the chemical lane approach their limiting value $\psi_{\ell}(\Lambda \rightarrow \infty, \Xi=0, \Theta, \Delta)=-1=\omega_{\ell}(\Lambda \rightarrow \infty, \Xi=0, \Theta, \Delta)$, corresponding to the homogeneous case with $\left(a_{\ell}, b\right) \mathrm{BC}$.

\section{A. Derjaguin approximation}

Using the underlying assumption of additivity of the forces, within the DA $(\Delta \rightarrow 0)$ we find for the scaling functions of the critical Casimir force and of the critical Casimir potential [see Appendix B

$$
\begin{aligned}
& \psi_{\ell}(\Lambda, \Xi, \Theta, \Delta \rightarrow 0)= \\
& 1+\psi_{\left(a_{\ell} \mid a, b\right)}(\Xi+\Lambda, \Theta, \Delta \rightarrow 0)-\psi_{\left(a_{\ell} \mid a, b\right)}(\Xi-\Lambda, \Theta, \Delta \rightarrow 0)
\end{aligned}
$$


and

$$
\begin{aligned}
& \omega_{\ell}(\Lambda, \Xi, \Theta, \Delta \rightarrow 0)= \\
& 1+\omega_{\left(a_{\ell} \mid a, b\right)}(\Xi+\Lambda, \Theta, \Delta \rightarrow 0)-\omega_{\left(a_{\ell} \mid a, b\right)}(\Xi-\Lambda, \Theta, \Delta \rightarrow 0),
\end{aligned}
$$

respectively. Thus, within the $\mathrm{DA}$, from the knowledge of the scaling functions $\psi_{\left(a_{\ell} \mid a, b\right)}$ [Eq. [16)] and $\omega_{\left(a_{\ell} \mid a, b\right)}$ [Eq. [18] ] for the chemical step with the appropriate $\mathrm{BC}$, one can directly calculate the corresponding scaling functions for the chemical lane configuration. Accordingly, in the limit $\Delta \rightarrow 0$ and for symmetry breaking $\mathrm{BC}, \psi_{\ell}$ and $\omega_{\ell}$ can be analytically calculated on the basis of Eqs. (29) and (30) by taking advantage of Eqs. (17), (19), and (20).

\section{B. Scaling function for the critical Casimir potential}

In Fig. 4(a) we show the scaling function $\omega_{\ell}$ for the critical Casimir potential obtained within the DA for $d=3$ and $d=4$ (MFT) at the bulk critical point $T=T_{c}$ [Eqs. (30) and [19] ] for various values of $\Lambda=L / \sqrt{R D}$ as a function of the lateral coordinate of the colloid. One can infer from Fig. 4 that, at bulk criticality, the critical Casimir potential varies less pronounced in $d=3$ than in $d=4$. As expected, for small values of $\Lambda$ (i.e., "narrow" chemical lanes), the potential does not reach the limiting homogeneous value -1 in the center of the chemical lane. On the other hand for large values of the scaling variable $\Lambda$ (i.e., "broad" chemical lanes), $\omega_{\ell}$ does attain the value -1 in the center of the chemical lane and the critical Casimir potential flattens. In this case the potential is adequately described by two independent chemical steps. However, the criterion for being a sufficiently "broad" lane depends sensitively on $\Theta$ and $d$. Indeed, from Eqs. (30) and (19) we find that at criticality $(\Theta=0)$ the critical Casimir potential at the center of the chemical lane $(\Xi=0)$ reaches the limiting value corresponding to the colloid facing a homogeneous substrate by up to $1 \%$ for $\Lambda \gtrsim 3.3$ in $d=4$ and for $\Lambda \gtrsim 10$ in $d=3$. We note that the curves in Fig. 4(a) as well as these bounds are independent of the actual boundary conditions because for all kinds of $\mathrm{BC}$ the scaling function of the normal critical Casimir force is constant at the critical point [see Eq. (2)].

Below we shall discuss some properties which are specific for BC with $a, a_{\ell}, b \in\{+,-\}$, which exhibit the feature that the normal critical Casimir force $f_{(\mp,-)}$ acting on two planar walls decays purely exponentially [see the text preceding Eq. (3)] as a function of their distance expressed in units of the bulk correlation length [see Eqs. (1) and (3)]. In Fig. 4(b) the scaling functions $\omega_{\ell}$ in $d=3$ and $d=4$ obtained from Monte Carlo simulation data ${ }^{52}$ and analytic MFT results 35 , respectively, within the DA [see Eqs. (30) and (20)] are shown for the same values of $\Lambda$ as in Fig. 4(a) but off criticality. For $\Theta=7.7$ the curves for $d=3$ and $d=4$ are indistinguishable from each other and from their common asymptotic expression given in Eq. (20) [see also Ref. 31]. For $\Theta \gg 1$, the critical Casimir potential attains its limiting homogeneous value in the center of the lane for values of $\Lambda$ which are smaller than the ones for $\Theta=0$ due to the shorter range of the forces. That is, for both $d=3$ and $d=4$ the single chemical lane is almost equally well approximated by two independent chemical
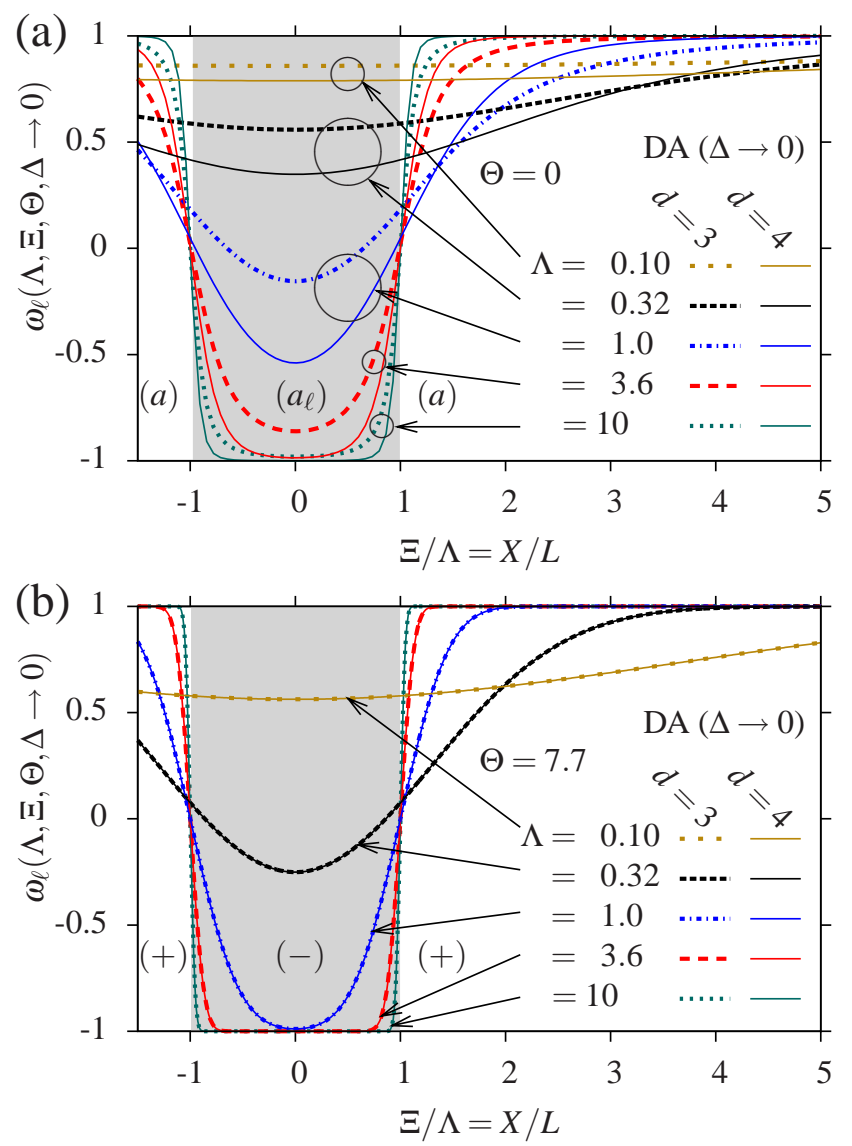

FIG. 4. Scaling function $\omega_{\ell}$ [Eq. 28)] describing the lateral variation of the critical Casimir potential of a colloid across a single chemical lane of width $2 L$ as a function of the lateral position $X$ of the colloid in units of the half width of the lane [see Fig. 1] $\Xi=X / \sqrt{R D}$, $\left.\Lambda=L / \sqrt{R D}, \Theta=D / \xi_{+}\right]$. Here, $\omega_{\ell}$ has been obtained within the DA $(\Delta \rightarrow 0)$ in $d=3$ and 4 [Eq. 30] ]. In (a) the curves correspond to $\Theta=0$ [Eq. 19]], whereas in (b) they correspond to $\Theta=7.7$ and $a, a_{\ell}, b \in\{+,-\}$ BC [Fig. 1]. For $\Theta \gg 1$ [(b)] the corresponding scaling functions obtained from Monte Carlo simulation data ${ }^{52}$ in $d=3$ and from analytic MFT results 35 in $d=4$ de facto coincide and their asymptotic expressions are given by Eqs. (20) and 30 . $\omega_{\ell}=1$ corresponds to the laterally homogeneous critical Casimir potential for $(a, b) \mathrm{BC}$ outside the chemical lane, whereas $\omega_{\ell}=-1$ corresponds to the value of the critical Casimir potential for the homogeneous case with $\left(a_{\ell}, b\right) \mathrm{BC}$ as within the chemical lane. For large values of $\Lambda$ the critical Casimir potential is the same as for two independent chemical steps, and $\omega_{\ell}$ reaches its limiting value -1 in the center of the lane at $\Xi=0$ [see the main text]. In (b), for $\Theta \gg 1$, $\omega_{\ell}$ attains -1 in the center of the chemical lane already for smaller values of $\Lambda$ due to the exponential decay of the critical Casimir force. We note that the DA results for $\Theta=0$ (i.e., at the critical point) are independent of the actual boundary conditions which, accordingly, were not specified in (a).

steps for $\Lambda \gtrsim 1.5$ at $\Theta=3.3$ (data not shown) and for $\Lambda \gtrsim 1.0$ at $\Theta=7.7$ [Fig. 4 (b)].

In Fig. 5] we compare the MFT $\omega_{\ell}$ obtained within the DA $(\Delta \rightarrow 0)$ at $\Theta=0$ [Eqs. (30) and (19)] with the scaling function obtained from the full numerical MFT calculations for $\Delta=1 / 3$. We find a rather good agreement even for small values of $\Lambda$ (i.e., "narrow" chemical lanes). This shows that for the geometry of a colloid close to a single chemical lane, nonlinearities, which are actually present in the critical Casimir 


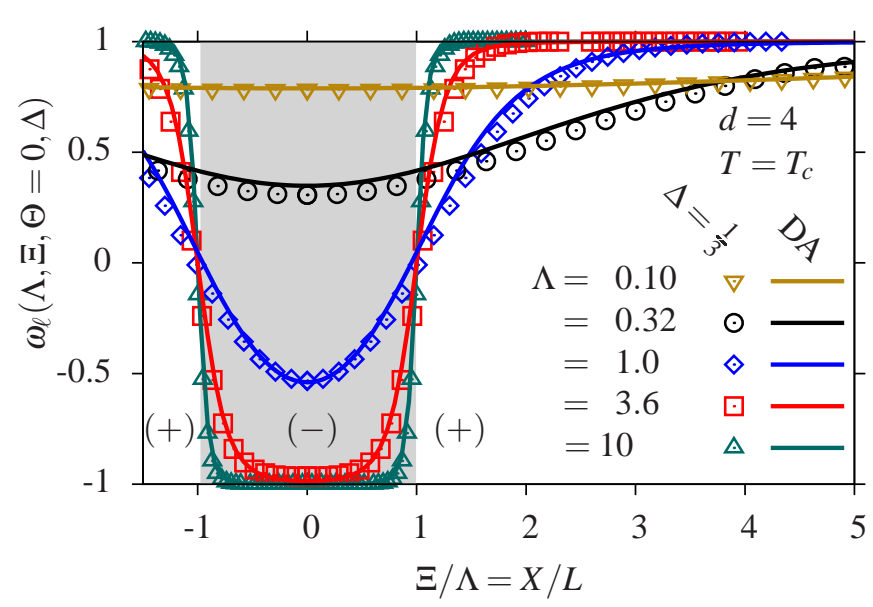

FIG. 5. Test of the performance of the DA for the scaling function $\omega_{\ell}$ [Eq. [28] of the critical Casimir potential for a sphere with (-) BC close to a single chemical lane with $(-) \mathrm{BC}$ embedded in a substrate with $(+)$ BC. The MFT $\omega_{\ell}$ is evaluated at bulk criticality $\Theta=0$ in $d=4$ both on the basis of the DA (lines, $\Delta \rightarrow 0$ ) and of the full numerical MFT (symbols, $\Delta=1 / 3$ ). There is good agreement between the DA and the full MFT results, even for small values of $\Lambda=L / \sqrt{R D}$. Nonlinear effects, which are inherently present in the theory, do not strongly affect the potential. For $\Delta \rightarrow 0$ the assumption of additivity of the critical Casimir forces underlying the DA is reliable even for small $\Lambda$.

effect and potentially invalidate the assumption of additivity underlying the DA, do not affect the resulting potential for small values of $\Delta$. We expect this property to hold beyond MFT in $d=3$ as well, in particular off criticality, i.e., for $\Theta \neq 0$.

\section{PERIODIC CHEMICAL PATTERNS (p)}

In this section we consider a pattern of chemical stripes which are alternating periodically along the $x$ direction. The pattern consists of stripes of width $L_{1}$ with $\left(a_{1}\right)$ BC joined with stripes of width $L_{2}$ with $\left(a_{2}\right) \mathrm{BC}$, such that the periodicity is given by $P=L_{1}+L_{2}$. Thus, the geometry of the substrate pattern is characterized by the two variables $L_{1}$ and $P$ [see Fig. 1]. The coordinate system is chosen such that the lateral coordinate $X$ of the center of the sphere is zero at the center of a $\left(a_{1}\right)$ stripe. The normal critical Casimir force $F_{\mathrm{p}}$ acting on the colloidal particle and its corresponding potential $\Phi_{\mathrm{p}}$ take on the following scaling forms:

$$
F_{\mathrm{p}}\left(L_{1}, P, X, D, R, T\right)=k_{B} T \frac{R}{D^{d-1}} K_{\mathrm{p}}(\lambda, \Pi, \Xi, \Theta, \Delta)
$$

and

$$
\Phi_{\mathrm{p}}\left(L_{1}, P, X, D, R, T\right)=k_{B} T \frac{R}{D^{d-2}} \vartheta_{\mathrm{p}}(\lambda, \Pi, \Xi, \Theta, \Delta),
$$

where $\Pi=P / \sqrt{R D}$ is the scaling variable characterizing the periodicity of the pattern and $\lambda=L_{1} / P$ is the scaling variable chosen to correspond to the relative width of the stripe with $\left(a_{1}\right)$ BC. $K_{\mathrm{p}}$ and $\vartheta_{\mathrm{p}}$ are universal scaling functions for the normal critical Casimir force and the critical Casimir potential, respectively. For $\lambda=1$ or 0 the force and the potential correspond to the homogeneous cases with $\left(a_{1}, b\right) \mathrm{BC}$ or $\left(a_{2}, b\right) \mathrm{BC}$, respectively [see Sec. III]. As before it is useful to define scaling functions $\psi_{\mathrm{p}}$ and $\omega_{\mathrm{p}}$ which vary for $\lambda \in[0,1]$ within the range $[-1,1]$ and describe the lateral behavior of the critical Casimir effect:

$$
\begin{aligned}
K_{\mathrm{p}}(\lambda, \Pi, \Xi, \Theta, \Delta) & =\frac{K_{\left(a_{2}, b\right)}+K_{\left(a_{1}, b\right)}}{2} \\
& +\frac{K_{\left(a_{2}, b\right)}-K_{\left(a_{1}, b\right)}}{2} \psi_{\mathrm{p}}(\lambda, \Pi, \Xi, \Theta, \Delta)
\end{aligned}
$$

and

$$
\begin{aligned}
\vartheta_{\mathrm{p}}(\lambda, \Pi, \Xi, \Theta, \Delta) & =\frac{\vartheta_{\left(a_{2}, b\right)}+\vartheta_{\left(a_{1}, b\right)}}{2} \\
& +\frac{\vartheta_{\left(a_{2}, b\right)}-\vartheta_{\left(a_{1}, b\right)}}{2} \omega_{\mathrm{p}}(\lambda, \Pi, \Xi, \Theta, \Delta) .
\end{aligned}
$$

\section{A. Derjaguin approximation}

Taking advantage of the assumption of additivity of the forces underlying the DA, one finds for the scaling function of the normal critical Casimir force in the limit $\Delta \rightarrow 0$ [see Appendix C

$$
\begin{aligned}
& \psi_{\mathrm{p}}(\lambda, \Pi, \Xi, \Theta, \Delta \rightarrow 0)= \\
& 1+\sum_{n=-\infty}^{\infty}\left\{\psi_{\left(a_{1} \mid a_{2}, b\right)}\left(\Xi+\Pi\left(n+\frac{\lambda}{2}\right), \Theta, \Delta \rightarrow 0\right)\right. \\
& \left.\quad-\psi_{\left(a_{1} \mid a_{2}, b\right)}\left(\Xi+\Pi\left(n-\frac{\lambda}{2}\right), \Theta, \Delta \rightarrow 0\right)\right\} .
\end{aligned}
$$

Thus, the knowledge of the scaling function $\psi_{\left(a_{1} \mid a_{2}, b\right)}$ for a single chemical step with the appropriate BC [Sec. IV] is sufficient to calculate directly the corresponding scaling function of the critical Casimir force acting on a colloid close to a periodic pattern of chemical stripes. As expected, from Eq. (35) one recovers the values $\psi_{\mathrm{p}}(\lambda=0, \Pi, \Xi, \Theta, \Delta)=1$ and $\psi_{\mathrm{p}}(\lambda=1, \Pi, \Xi, \Theta, \Delta)=-1$, i.e., the cases of a colloid with $(b) \mathrm{BC}$ facing a homogeneous substrate with $\left(a_{2}\right) \mathrm{BC}$ and $\left(a_{1}\right) \mathrm{BC}$, respectively [see Appendix C].

In the limit $\Pi \rightarrow 0$, i.e., for a pattern with a very fine structure compared to the size of the colloid, the sum in Eq. (35) turns into an integral [see Appendix $\mathrm{C}$ ] and, as expected, $\psi_{\mathrm{p}}$ becomes independent of $\Xi$, i.e., of the lateral position of the colloid:

$$
\psi_{\mathrm{p}}(\lambda, \Pi \rightarrow 0, \Xi, \Theta, \Delta \rightarrow 0)=1-2 \lambda
$$

Accordingly, in the limit $\Pi \rightarrow 0$ the force acting on the colloid - within the DA - is the average of the ones corresponding to the two boundary conditions weighted by the corresponding relative stripe width [see Eqs. (36) and (33)]:

$$
\begin{aligned}
& K_{\mathrm{p}}(\lambda, \Pi \rightarrow 0, \Xi, \Theta, \Delta \rightarrow 0)= \\
& \frac{L_{1}}{L_{1}+L_{2}} K_{\left(a_{1}, b\right)}(\Theta, \Delta \rightarrow 0)+\frac{L_{2}}{L_{1}+L_{2}} K_{\left(a_{2}, b\right)}(\Theta, \Delta \rightarrow 0) .
\end{aligned}
$$

For the scaling function of the critical Casimir potential the results are completely analogous to Eqs. (35)-37] [see Appendix $\mathrm{C}$. 

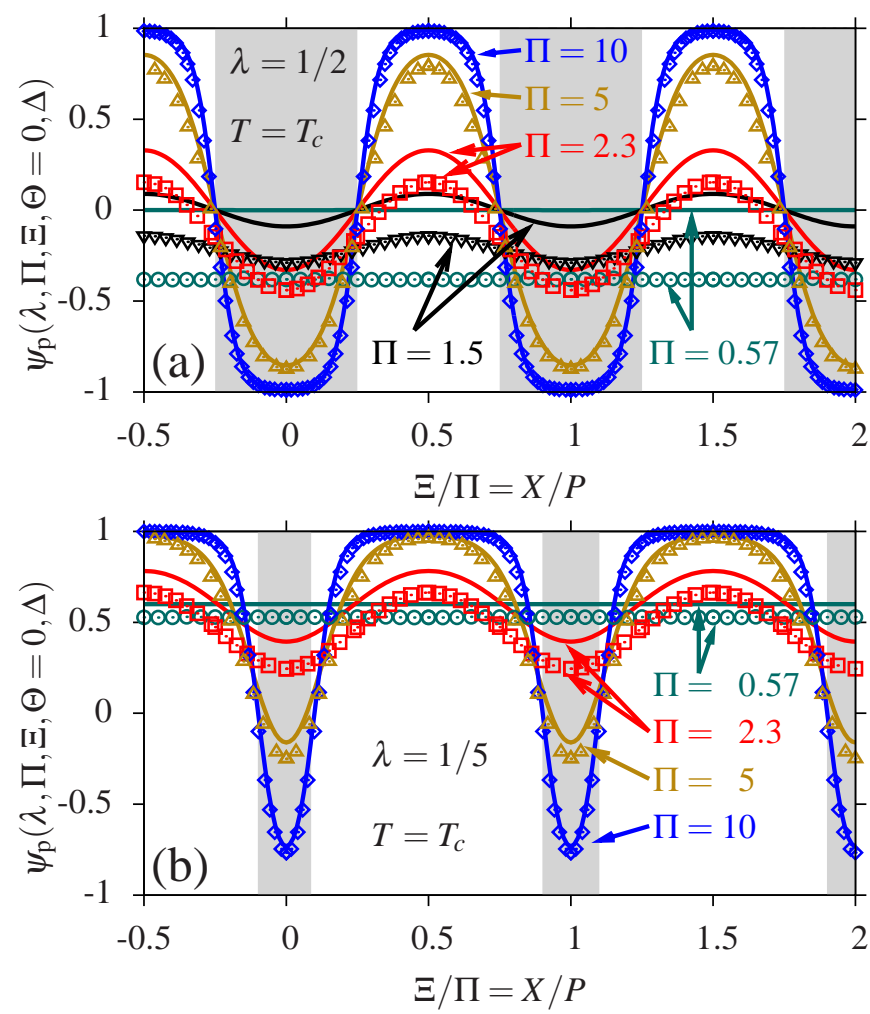

FIG. 6. MFT $(d=4)$ scaling function $\psi_{\mathrm{p}}$ [Eq. 33] of the normal critical Casimir force acting on a colloidal sphere with $(b)=(-)$ $\mathrm{BC}$ which is close to a periodically patterned substrate [Fig. 1] with $\left(a_{1}\right)=(-) \mathrm{BC}$ on one kind of stripes [shaded areas] and $\left(a_{2}\right)=(+)$ $\mathrm{BC}$ on the other kind of stripes. Due to this choice of the $\mathrm{BC}$ the colloid is attracted by the shaded stripes and repelled by the others. $\psi_{\mathrm{p}}$ is shown as a function of the lateral position of the colloid $X / P$ with $P=L_{1}+L_{2}$ and at the bulk critical point $\Theta=0$. The geometry of the pattern is characterized by $\Pi=P / \sqrt{R D}$ and $\lambda=L_{1} / P$, for which we have chosen the values (a) $\lambda=0.5$ and (b) $\lambda=0.2$. The lines are the results for $\psi_{\mathrm{p}}$ as obtained within the DA for $d=4$ [Eqs. (35) and [17]], whereas the symbols represent the full numerical data obtained within MFT for $\Delta=1 / 3$ for various values of $\Pi$. For patterns which are finely structured on the scale of the colloid size, i.e., $\Pi \lesssim 2$, the actual results deviate from the approximate ones obtained within the DA due to the strong influence (in this context) of the inherent nonlinear effects.

\section{B. Scaling function for the normal critical Casimir force}

Figure 6] shows the scaling function $\psi_{\mathrm{p}}$ [Eq. [33] ] as a function of $\Xi / \Pi=X / P$, describing the lateral variation of the normal critical Casimir force at $\Theta=0$ as obtained within the DA for $d=4$ [Eq. (35) with Eq. (17); solid lines] compared with the one obtained from the full numerical MFT calculation [ $\Delta=1 / 3$; symbols] for symmetry breaking boundary conditions $\left(a_{1}\right)=(-),\left(a_{2}\right)=(+)$, and $(b)=(-)$ [Fig. 1]. From this comparison for $\lambda=0.5$ [Fig.6 (a)] and $\lambda=0.2$ [Fig.6(b)] and for various values of $\Pi$ one can infer that for $\Delta \rightarrow 0$ and $\Pi \gg 1$, i.e., $L_{1}+L_{2} \gg \sqrt{R D}$ the DA describes well the actual behavior of the scaling function, even if the force scaling function does not attain its limiting homogeneous values $\psi_{\mathrm{p}}= \pm 1$ in the center of the stripes. However, for $\Pi \lesssim 2$ (in $d=4$ at $T=T_{c}$ ) the DA does not quantitatively describe the actual behavior and the scaling function $\psi_{\mathrm{p}}$ obtained from
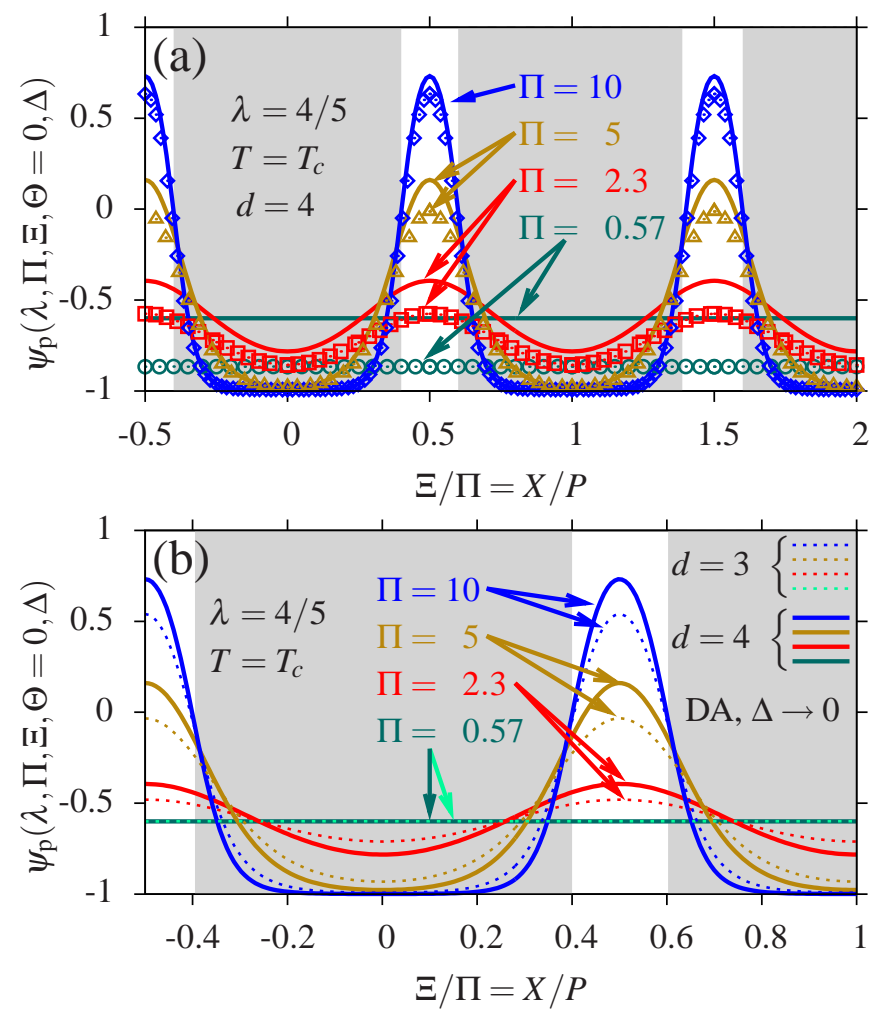

FIG. 7. (a) The same as in Fig. 6 but for $\lambda=0.8$. Also in this case, the DA turns out to be accurate for $\Pi \gtrsim 2$ while it fails to describe quantitatively the full numerical data for smaller values of $\Pi$. (b) Comparison between the scaling functions $\psi_{\mathrm{p}}$ in $d=3$ (dotted lines) and $d=4$ (solid lines), at $T=T_{c}$, for $\lambda=0.8$, and within the DA. At the critical point the expression for this scaling function $\psi_{\mathrm{p}}$ is known analytically [see Eqs. (35) and (17)], and the corresponding plot presented here shows that the lateral variation of the normal critical Casimir force is less pronounced in $d=3$ than in $d=4$. (We note that for $\Pi \rightarrow 0$ we expect that also in $d=3$ the DA fails to describe quantitatively the actual behavior; however, we nonetheless present the curve for $\Pi=0.57$ in order to show that the critical Casimir force obtained within the DA practically does not change laterally for such small values of П.)

the full numerical MFT calculations deviates from the one obtained within the DA. Within both the DA and the full numerical MFT calculation, for $\Pi \rightarrow 0$ the normal critical Casimir force loses its lateral dependence on $\Xi$. But from the full numerical calculation we find that the corresponding constant value which is attained by $\psi_{\mathrm{p}}$ differs from the one obtained within DA [Eq. (36)]. This shows that for small periodicities $P \lesssim \sqrt{R D}$ nonlinearities inherent in the critical Casimir effect strongly affect the resulting scaling functions of the force and the potential, so that in this respect the assumption of additivity of the force and thus the use of the DA are not justified.

Figure 7 (a) shows the same comparison as Fig. 6 but for $\lambda=0.8$, which corresponds to an areal occupation of $80 \%$ of the substrate surface with $(-)$ BC and $20 \%$ with $(+)$ BC. Due to the fact that at the critical point $\psi_{\left(a_{1} \mid a_{2}, b\right)}(\Xi, \Theta=0, \Delta \rightarrow 0)$ is actually independent of the $\mathrm{BC}, \psi_{\mathrm{p}}(\lambda=0.8, \Pi, \Xi, \Theta=$ $0, \Delta \rightarrow 0)$ in Fig. 7(a) is, within the DA, complementary to the one for $\lambda=0.2$ in Fig. 6(b), i.e., it is obtained from the latter by a reflection with respect to $\psi_{\mathrm{p}}=0$ followed by a shift in $\Xi / \Pi$ of 0.5 . Instead, the full numerical data in Fig.77a) and 


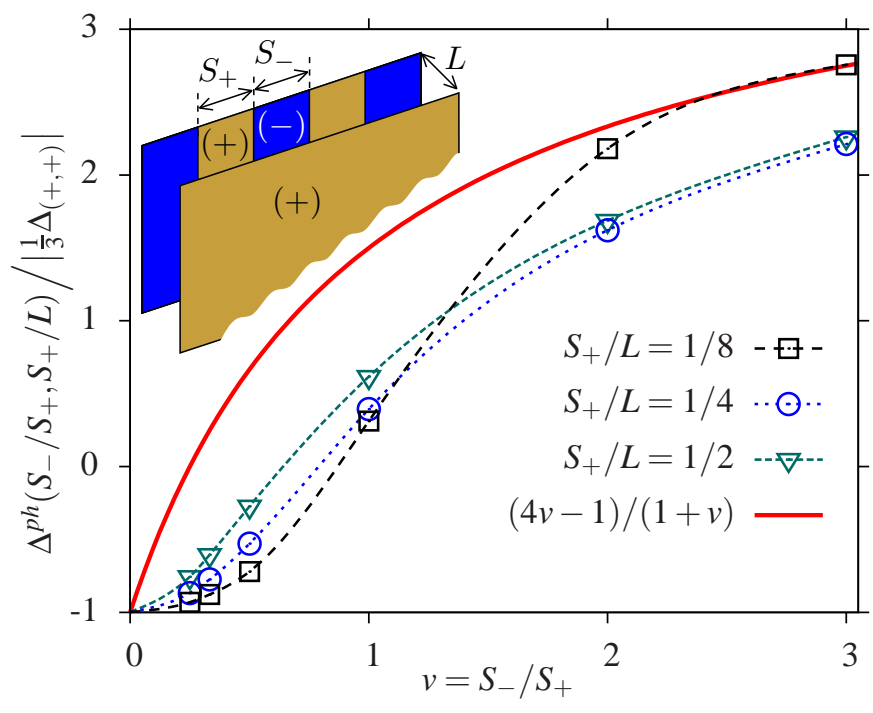

FIG. 8. Normalized scaling function $\Delta^{p h}$ of the critical Casimir force at criticality acting on a homogeneous planar wall with $(+)$ BC opposite to a periodically patterned planar substrate with stripes of alternating $(+)$ and $(-) \mathrm{BC}$ as a function of $v=S_{-} / S_{+}$, where $S_{+}$and $S_{-}$are the respective widths. The symbols correspond to the MFT $(d=4)$ data presented in Fig. 12 of Ref. 33 for various values of $S_{+} / L$ (note that $\Delta_{0}^{++}=\Delta_{(+,+)} /(d-1)$ in Fig. 12 of Ref. 33). The dashed and dotted lines which join the data points are a guide to the eye. The solid line corresponds to the DA result given in Eq. (41) which assumes additivity of the forces and turns out to be independent of the ratio $S_{+} / L$. One can immediately infer from the graph that here the assumption of additivity is not justified, which is the limiting configuration of the sphere-wall geometry for $\Pi \rightarrow 0$.

Fig. 6(b) show a different behavior as they clearly tend to assume the value -1 corresponding to the homogeneous case with $(-,-)$ BC. By contrast, for the case $\lambda=0.2$ shown in Fig. 6b), the full numerical data do not reach as closely the value +1 corresponding to $(+,-) \mathrm{BC}$, although the substrate area is covered by $80 \%$ with $(+) \mathrm{BC}$. This feature is addressed in more detail in Sec. $\mathrm{VII}$ Figure 7 b) compares the scaling function $\psi_{\mathrm{p}}$ of the normal critical Casimir force at $T=T_{c}$ and for $\lambda=0.2$ as obtained within the DA for $d=4$ (solid lines) with the corresponding one for $d=3$ (dotted lines). At $T=T_{c}$, $\psi_{\mathrm{p}}$ is determined by Eqs. (35) and (17) from which one can infer that the lateral variation of the normal Casimir force is less pronounced for $d=3$ than for $d=4$. This qualitative feature holds for all values of $\lambda$ (not shown). However, off criticality, $\Theta \gg 1$, [according to Eqs. (35) and (20)] the DA scaling functions both for $d=3$ as obtained from MC simulation data and for $d=4$ as obtained from MFT de facto coincide (not shown), similarly to the case of a single chemical lane in Fig. 4(b).

Although one would expect the DA to be valid for large radii $R$, the lateral variation of the boundary conditions at the surface of the patterned substrate on a scale $P \lesssim \sqrt{R D}$ - corresponding to the limit $\Pi \rightarrow 0$ - renders the DA less accurate, as it clearly emerges from the numerical data presented in Figs. 6 and7. The fact that a large colloid radius $R$ does not guarantee the validity of the DA can be understood by noting that such a discrepancy between the full numerical calculation and the result of the DA approximation already emerges in the film geometry (formally corresponding to the limit $R \rightarrow \infty$ ), i.e., for a chemically $p$ atterned wall opposite to a laterally homogeneous flat wall. This " $p h$ " configuration has been studied in Ref. 33 within MFT for laterally alternating chemical stripes of width $L_{1}=S_{+}$and $L_{2}=S_{-}$with $(+)$and $(-)$BC, respectively, opposite to a homogeneous substrate with $(+) \mathrm{BC}$ a distance $L$ apart [see Fig. 1] and the inset of Fig. 8]. Indeed, by using the assumption of additivity of the critical Casimir forces underlying the DA and neglecting edge effects, the normal critical Casimir force $f_{(\mathrm{DA})}^{p h}\left(S_{+}, S_{-}, L, T\right)$ per unit area acting on the walls is predicted to be given by

$$
\begin{aligned}
f_{(\mathrm{DA})}^{p h}\left(S_{+}, S_{-}, L, T\right)= & \\
& \quad \frac{S_{+}}{S_{+}+S_{-}} f_{(+,+)}(L, T)+\frac{S_{-}}{S_{+}+S_{-}} f_{(+,-)}(L, T),
\end{aligned}
$$

where $f_{(+, \pm)}$refer to homogeneous parallel walls, as in Eq. (1). At the bulk critical point the critical Casimir force is given in general by 33

$$
f^{p h}\left(S_{+}, S_{-}, L, T=T_{c}\right)=k_{B} T_{c} \frac{d-1}{L^{d}} \Delta^{p h}\left(v=\frac{S_{-}}{S_{+}}, \frac{S_{+}}{L}\right) .
$$

Using Eq. (38) together with Eqs. (1) and (2) one finds within the DA that

$$
(d-1) \Delta_{(\mathrm{DA})}^{p h}\left(v, \frac{S_{+}}{L}\right)=\frac{v \Delta_{(+,-)}+\Delta_{(+,+)}}{1+v},
$$

which renders the rhs of Eq. (40) to be independent of the scaling variable $S_{+} / L$. Within MFT as studied in Ref. $33(d=$ 4), one has $\Delta_{(+,-)}=-4 \Delta_{(+,+)}>0$ [see the end of Sec. [IIB] so that

$$
\Delta_{(\mathrm{DA})}^{p h}\left(v, \frac{S_{+}}{L}\right)=\frac{\left|\Delta_{(+,+)}\right|}{3} \frac{4 v-1}{1+v} .
$$

In Fig. 8 we show the comparison between the actual scaling function $\Delta^{p h}$ (data points, obtained numerically as reported in Fig. 12 of Ref. 33) and $\Delta_{(\mathrm{DA})}^{p h}$ (Eq. (41), solid line) derived by assuming additivity of the forces and neglecting edge effects. Figure 8 clearly shows that the actual behavior of the critical Casimir force in the film geometry is not properly predicted within these assumptions. This is expected to be due to the presence of nonlinear effects and of edge effects in this context. This explains why in the limit $\Pi \rightarrow 0$ the DA $(R \gg D)$ used here does not capture the behavior of the critical Casimir force acting on a colloid close to periodically patterned substrate.

In Fig. 9 we show the behavior of scaling function $K_{\mathrm{p}}$ [Eq. (31)] of the normal critical Casimir force acting on the colloid in $d=4$ with $(b)=(-) \mathrm{BC}$ as a function of $\Theta=D / \xi_{+}$ (i.e., as a function of the normal distance of the colloid from the substrate in units of the bulk correlation length) and for various values of $\lambda$ and $\Pi$. In Fig. 9 the scaling function $K_{\mathrm{p}}$ is evaluated at $X=0$ [see Fig. 1] which corresponds to the most preferred lateral position of the colloid in which the normal force is least repulsive or most attractive [see Fig. 6]. From Fig. 9 one can infer that the DA does not provide an accurate estimate of $K_{\mathrm{p}}$ in the whole range of $\Theta$ for $\Pi=0.57$ [panel (b)], whereas it does so for $\Pi=2.3$ [panel (a)]. Indeed, for $\Pi=0.57$ the discrepancy between the DA and the numerical data is already significant for $\Theta \lesssim 4$ and $0.3 \lesssim \lambda \lesssim 0.9$, 

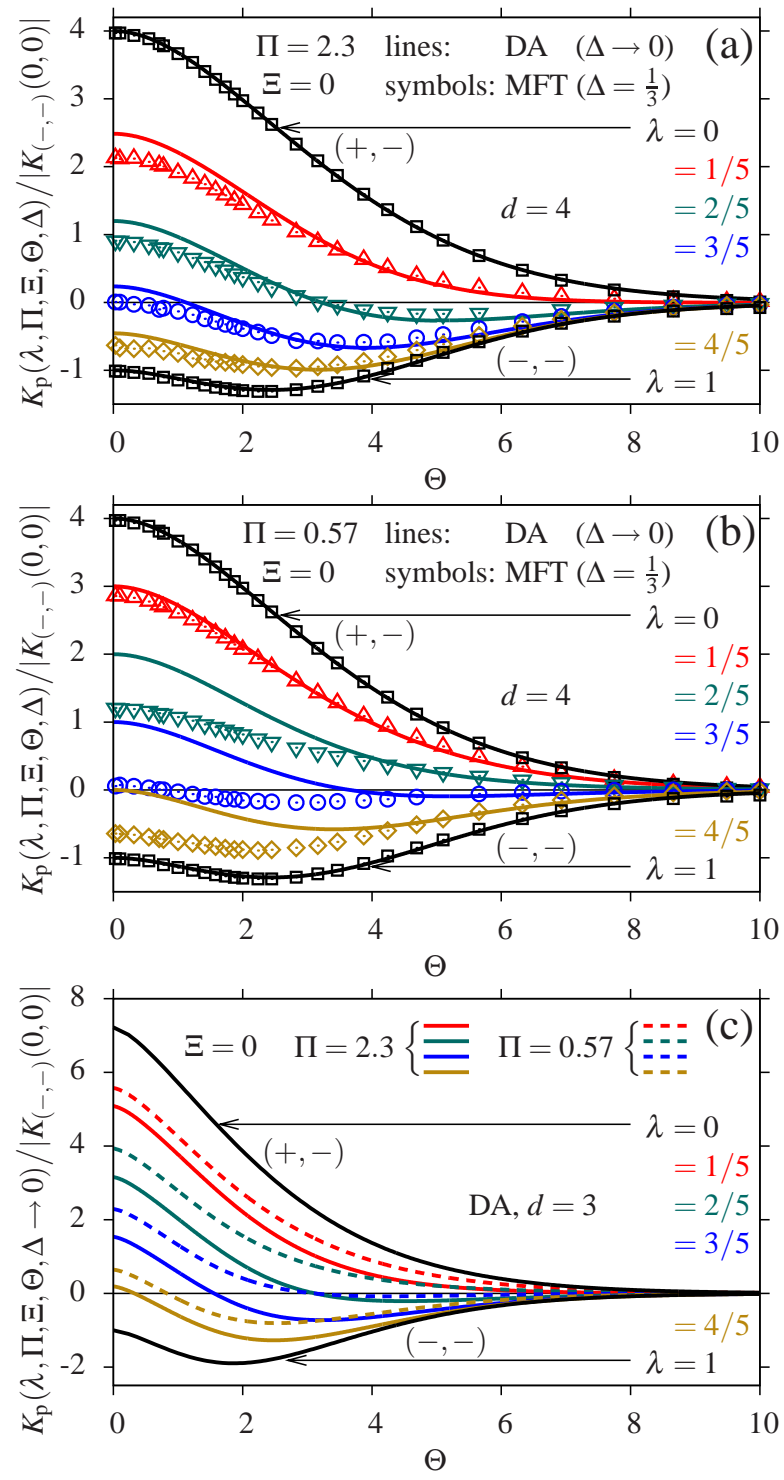

FIG. 9. Scaling function $K_{\mathrm{p}}$ [Eq. [31)] of the normal critical Casimir force acting on a spherical colloid with $(-)$ BC located at $X=0$ $(\Xi=X / \sqrt{R D})$ close to a periodically chemically patterned substrate [see Fig. 1]. $K_{\mathrm{p}}$ is suitably normalized by the absolute value of the force scaling function $K_{(-,-)}(0,0)=2 \pi \Delta_{(-,-)} /(d-1)$ for the homogeneous $(-,-)$ case at criticality and within the DA [Sec. IIIA]. The lateral position of the center of the colloid is fixed at the center of a stripe with $\left(a_{1}\right)=(-) \mathrm{BC}$ and width $L_{1}=\lambda P$, which it is attracted to, in contrast to the second type of stripes with $\left(a_{2}\right)=(+)$ $\mathrm{BC}$ and width $L_{2}=(1-\lambda) P$, which it is repelled from. The scaling variable corresponding to the periodicity of the substrate pattern is (a) $\Pi=P / \sqrt{R D}=2.7$ and (b) $\Pi=0.57$, whereas the relative area fraction of the $(-)$ stripes changes from $\lambda=L_{1} /\left(L_{1}+L_{2}\right)=0$ to $\lambda=1$ (top to bottom: fully repulsive to fully attractive). In (a) and (b) the lines represent the result for the MFT critical Casimir force within the DA [ $\Delta \rightarrow 0, d=4$, see Eq. [35]], whereas the symbols represent the full numerical MFT data obtained for $\Delta=1 / 3$. The DA agrees reasonably well with the full data for $\Pi=2.3[$ (a) $]$ and $\Theta \gtrsim 1$, but for $\Pi=0.57$ [(b)] it fails to describe the actual behavior within the ranges $\Theta \lesssim 4$ and $0.3 \lesssim \lambda \lesssim 0.9$ where the nonlinear effects strongly affect the resulting scaling function. In (c) $K_{\mathrm{p}}$ is shown for $\Pi=0.57$ and 2.3, as obtained for $d=3$ within the DA on the basis of the Monte Carlo simulation data for the film geometry 52 . (We note, however, that we do not expect that the curves shown for $\Pi=0.57$ are quantitatively reliable.) whereas for $\Pi=2.3$ agreement is found for all values of $\lambda$ except for $\Theta \lesssim 1$ [Fig. 9 (a)]. This fact suggests that for relatively small periodicities $\Pi \lesssim 2$ non-additive and edge effects become important. On the other hand, for large values of $\Theta \gg 1$ the DA describes the behavior of $K_{\mathrm{p}}$ rather well for all values of $\Pi$ due to the exponential decay of the critical Casimir force for $\Theta \gg 1$ [Eq. (3)]. Figure 9 (c) shows the scaling function $K_{\mathrm{p}}$ for $d=3$ within the DA as obtained from Monte Carlo simulation data for the film geometry 52 . The qualitative features of the behavior of $K_{\mathrm{p}}$ in $d=3$ and $d=4$ are similar.

From our analysis in $d=4$ we conclude that the DA describes quantitatively well the behavior of the actual critical Casimir force for $\Pi \gtrsim 2$ for all values of $\Theta$. For smaller values of $\Pi$, the DA is only quantitatively reliable for large values of $\Theta$ (at which the force decays exponentially). For example, for $\Pi \gtrsim 0.5$ the DA result is quantitatively correct for $\Theta \gtrsim 4$. We expect these properties to be carried over to $d=3$.

\section{Critical Casimir levitation}

Rather remarkably, within a certain range of values of $\lambda$, $K_{\mathrm{p}}$ changes sign as a function of $\Theta=D / \xi_{+}$[Fig. 9]. In this context it is convenient to introduce for later purposes another scaling variable $\Psi=\Pi|\Theta|^{1 / 2}=P / \sqrt{R \xi_{ \pm}}$which is independent of $D$ and therefore does not vanish in the DA limit $D \ll R$ (i.e., $\Delta \rightarrow 0$ ). Due to this change of sign of $K_{\mathrm{p}}$, there exists a certain value $\Theta=\Theta_{0}(\Psi, \lambda, \Xi, \Delta)$ at which the normal critical Casimir force $F_{\mathrm{p}}$ acting on the colloid vanishes. This implies that in the absence of additional forces the colloid levitates at a height $D_{0}$ determined by $\Theta_{0}$ and $\xi_{+}$, which can be tuned by changing the temperature. Since for fixed geometrical parameters $R, X$, and $P$ the scaling variables $\Theta, \Pi, \Xi$, and $\Delta$ depend on $D$, one has to consider the behavior of $F_{\mathrm{p}}$ as a function of $D$ near $D_{0}$ in order to assess whether the levitation is stable against perturbations of $D$ or not. Stability requires $\left.\partial_{D} F_{\mathrm{p}}\right|_{D=D_{0}}<0$ (so that for $D<D_{0}$ the colloid is repelled from the patterned substrate, whereas for for $D>D_{0}$ it is attracted). According to Eq. (31) one has

$$
\begin{aligned}
& \partial_{D} F_{\mathrm{p}}=k_{B} T \frac{R}{D^{d}} \times\{-(d-1) \\
& \left.\quad-\frac{1}{2} \Pi \partial_{\Pi}-\frac{1}{2} \Xi \partial_{\Xi}+\Theta \partial_{\Theta}+\Delta \partial_{\Delta}\right\} K_{\mathrm{p}}(\lambda, \Pi, \Xi, \Theta, \Delta) .
\end{aligned}
$$

The laterally preferred position is always at $X=X_{0}=0$, corresponding to $\Xi=\Xi_{0}=0$, so that within the DA $(\Delta \rightarrow 0)$ one has

$$
\begin{aligned}
& \operatorname{sign}\left(\left.\partial_{D} F_{\mathrm{p}}\right|_{D=D_{0}, X=X_{0}, \mathrm{DA}}\right)= \\
& \operatorname{sign}\left(\left.\left\{-\frac{1}{2} \Pi \partial_{\Pi}+\Theta \partial_{\Theta}\right\} K_{\mathrm{p}}(\lambda, \Pi, \Xi=0, \Theta, \Delta \rightarrow 0)\right|_{\Theta=\Theta_{0}}\right),
\end{aligned}
$$

where we have used the implicit equation $\left.F_{\mathrm{p}}\right|_{D=D_{0}}=0$ so that $\left.K_{\mathrm{p}}\right|_{D=D_{0}}=0$. (Equation (43) assumes that $\partial_{\Delta} K_{\mathrm{p}}$ does not diverge $\propto \Delta^{-1}$ for $\Delta \rightarrow 0$.) In the following we only consider $\Theta \geq 0$ and $\mathrm{BC}\left(a_{1}\right)=(-),\left(a_{2}\right)=(+)$, and $(b)=(-)$.

Within the DA we find that both $\left.\partial_{\Pi} K_{\mathrm{p}}\right|_{\Theta=\Theta_{0}, \Xi=\Xi_{0}}$ and $\left.\partial_{\Theta} K_{\mathrm{p}}\right|_{\Theta=\Theta_{0}, \Xi=\Xi_{0}}$ are negative, so that according to Eq. (43) the

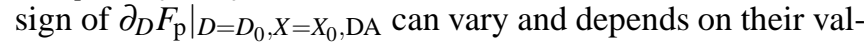
ues as well as on $\Theta_{0}$ and $\Pi$. However, at criticality $(\Theta=0)$ the 

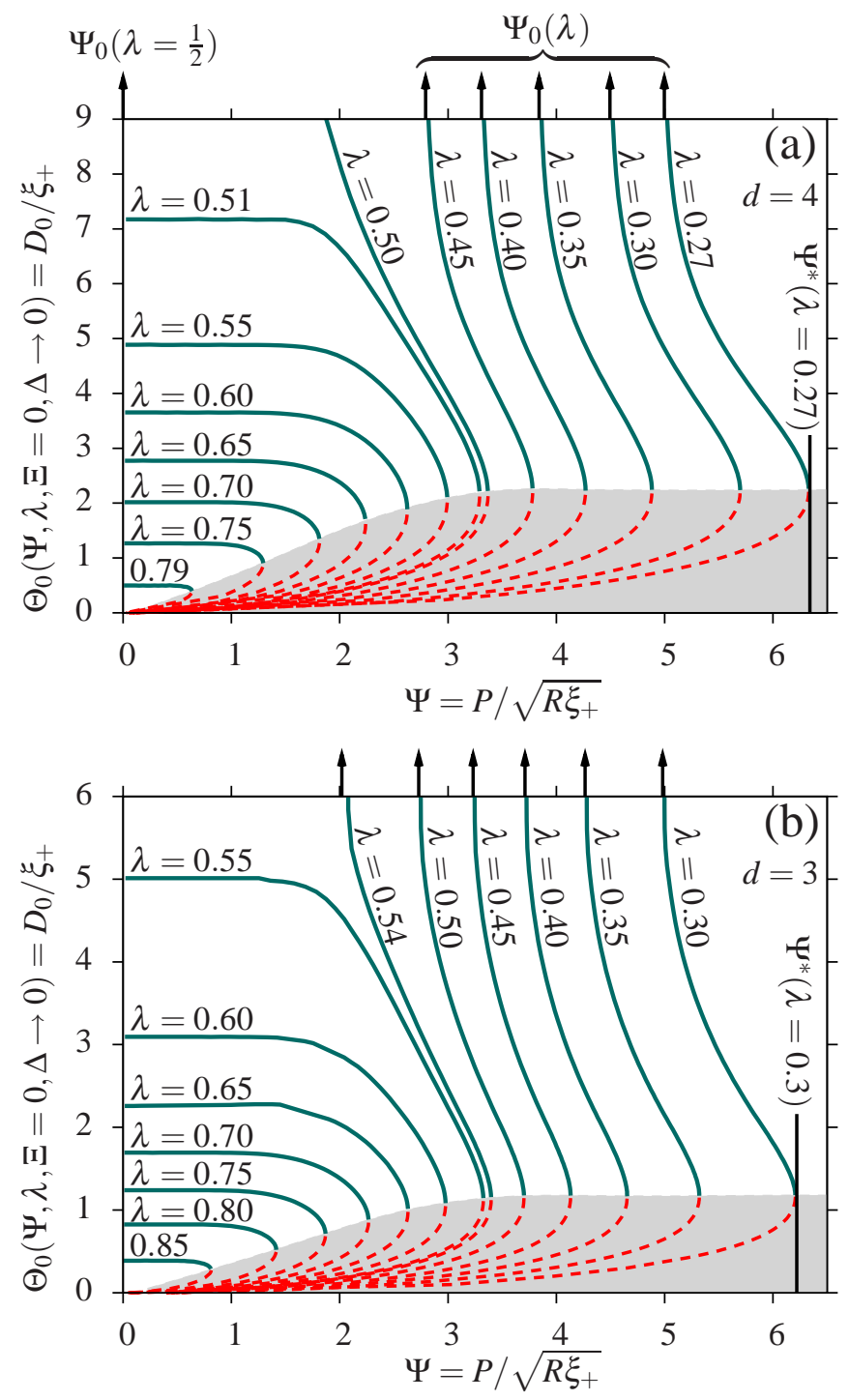

FIG. 10. Values of the scaling variable $\Theta_{0}$ at which within the DA $(\Delta \rightarrow 0)$ the normal critical Casimir force $K_{\mathrm{p}}$ shown in Fig. 9 vanishes as a function of $\Psi$ for (a) $d=4$ and (b) $d=3$ on the basis of Monte Carlo simulation data ${ }^{52}$ and for various values of $\lambda=L_{1} / P$. The solid lines correspond to values of $\Theta_{0}$ for which the levitation of the colloid at a height $D_{0}$ above the substrate is stable against perturbations of $D\left[\left.\partial_{D} F_{\mathrm{p}}\right|_{D=D_{0}}<0\right.$, see Eq. [43] ]. The shaded region and the dashed lines indicate those values of $\Theta_{0}$ for which $\left.\partial_{D} F_{\mathrm{p}}\right|_{D=D_{0}}>0$ and thus do not correspond to stable levitation. For $\lambda>\lambda_{0}$ with $\lambda_{0}(d=4)=4 / 5$ and $\lambda_{0}(d=3) \simeq 0.88, \Theta_{0}$ ceases to exist, i.e., $K_{\mathrm{p}}$ does not exhibit a zero. For $\lambda<\lambda_{1}$ with $\lambda_{1}(d=4)=1 / 2$ and $\lambda_{1}(d=3) \simeq 0.545, \Theta_{0}\left(\Psi \searrow \Psi_{0}(\lambda)\right)$ diverges. (The values for $\Psi_{0}(\lambda)$ are indicated by upward arrows.) For any $\lambda<\lambda_{0}, \Theta_{0}$ exists for $\Psi<\Psi^{*}(\lambda)$. (From the analysis in Fig. 9 we expect the DA to be quantitatively reliable only for $\Psi \gtrsim 2 \sqrt{\Theta_{0}}$ for $\Theta_{0} \lesssim 4$ and for $\Psi \gtrsim 0.5 \sqrt{\Theta_{0}}$ for $\Theta_{0} \gtrsim 4$, which implies $\lambda \lesssim 0.7$ in $d=3$ and $\lambda \lesssim 0.6$ in $d=4$.)

second term of the rhs of Eq. (43) vanishes. Thus, at the bulk critical point $T=T_{c}$ the derivative $\partial_{D} F_{\mathrm{p}}$ evaluated at $D=D_{0}$ and $X=X_{0}=0$ is always positive so that one cannot achieve stable levitation. On the other hand, for $\Theta>0$ it is always possible to find geometrical configurations for which the colloid exhibits stable levitation, as described in the following.
Figure 10 shows the values of $\Theta_{0}$ at which the normal critical Casimir force acting on a colloid vanishes as a function of the new scaling variable $\Psi$ introduced at the beginning of this subsection, for various $\lambda$, for $\Xi=0$, and within the DA ( $\Delta \rightarrow 0$ ) for (a) $d=4$ and (b) $d=3$. The corresponding sign of $\left.\partial_{D} F_{\mathrm{p}}\right|_{D=D_{0}}$ [according to Eq. (43)] is also indicated: $\Theta_{0}$ drawn as a solid line indicates $\left.\partial_{D} F_{\mathrm{p}}\right|_{D=D_{0}}<0$, i.e., stable levitation of the colloid; a dashed line, instead, indicates $\left.\partial_{D} F_{\mathrm{p}}\right|_{D=D_{0}}>0$ and therefore a local maximum of the critical Casimir potential with respect to $D$, which occurs within the shaded regions in Fig. 10. For a given value of $\lambda$ (with $\lambda_{1}<\lambda<\lambda_{0}$ as we shall discuss in detail further below), e.g., $\lambda=0.60$ in Fig. 10,a), the corresponding curve for $\Theta_{0}$ shows a bifurcation at $\Psi=\Psi^{*}(\lambda)$ such that a vertical line drawn in Fig. 10 at a certain $\Psi$ intersects this curve in two points $\Theta_{0, u}$ and $\Theta_{0, s}>\Theta_{0, u}$ if $\Psi<\Psi^{*}(\lambda)$, whereas it has no intersection for $\Psi>\Psi^{*}(\lambda)$. In the former case $\Theta_{0, u}$ and $\Theta_{0, s}$ correspond to a local maximum and to a local minimum of the critical Casimir potential at distances $D_{0, u}=\xi_{+} \Theta_{0, u}$ and $D_{0, s}=\xi_{+} \Theta_{0, s}$, respectively, i.e., to an $u$ nstable and a stable levitation point for the colloid, respectively. Instead, for $\Psi>\Psi^{*}(\lambda)$, the critical Casimir force has no zero at any finite value of $D$. We note that $D=0$ (stiction) and thus $\Theta=0$ always corresponds to the global minimum of the potential because for $D \rightarrow 0$ the critical Casimir potential is strongly attractive. The corresponding geometrical configuration into which the colloid is finally attracted by the substrate [due to $\left(a_{1}\right)=(-),(b)=(-)$, and $X=0$, see Fig.1] is stabilized by the steric repulsion of the wall. We note that within the DA the critical Casimir potential for $X=0$ is attractive at sufficiently small distances, even if the major part of the substrate is characterized by $(+) \mathrm{BC}$, i.e., even if $0 \neq \lambda \ll 1$. Indeed, in this case the potential of the colloid at $X=0$ and close to a periodically patterned substrate can be approximated by the one due to a single chemical lane centered at $X=0$, which has been discussed in Sec. V. For given colloid radius $R$ and width $L_{1}=\lambda P>0$ of the attractive stripe, the scaling variable $\Lambda=L_{1} /(2 \sqrt{R D})$ diverges as $D \rightarrow 0$, so that the scaling function $\omega_{\ell}(\Lambda, \Xi, \Theta, \Delta)$ which characterizes the potential of the lane [see Eq. (28)] attains the value -1 corresponding to the case of homogeneous, attractive $(-,-)$ BC [see Fig. 4]. Within this approximation and for $D \ll \xi_{ \pm}$ the critical Casimir force becomes attractive if $\vartheta_{\mathrm{p}} \simeq \vartheta_{\ell}<0$ which, due to Eqs. (28), (11), and (2), yields the condition $\omega_{\ell}(\Lambda, \Xi=0, \Theta \rightarrow 0, \Delta \rightarrow 0)<1-2 \Delta_{(+,-)} /\left(\Delta_{(+,-)}-\Delta_{(+,+)}\right)$, i.e., $\omega_{\ell}<-0.6$ in $d=4^{35}$ and $\omega_{\ell} \lesssim-0.76$ in $d=352$; this occurs for $\Lambda>\Lambda_{0}=1.1$ in $d=4$, and $\Lambda>\Lambda_{0}=2.7$ in $d=3$, respectively [see also Fig. 4(a)]. Accordingly, at distances $D<\lambda^{2} P^{2} /\left(4 R \Lambda_{0}^{2}\right)$ (together with $\left.D \ll \xi_{ \pm}\right)$the critical Casimir potential $\Phi_{\mathrm{p}}$ is negative and diverges to $-\infty$ for $D \rightarrow 0$. (However, for very small values of $\lambda$ this would occur at distances of microscopic scale such that the scaling limit and thus the form of $\Phi_{\mathrm{p}}$ do no longer hold). Thus the bifurcation of $\Theta_{0}$ at $\Psi^{*}(\lambda)$ corresponds to a transition from (metastable) levitation at $D=D_{0, s}$ for $\Psi<\Psi^{*}(\lambda)$ to stiction at $D=0$ for $\Psi>\Psi^{*}(\lambda)$. For $\Psi<\Psi^{*}(\lambda)$ the metastable levitation minimum at $D_{0, s}$ is shielded from the global minimum at $D=0$ by a potential barrier the height of which vanishes for $\Psi \nearrow \Psi^{*}(\lambda)$ [see Fig. 11]. Experimentally, one typically varies the value of $\xi_{+}$by changing the temperature ${ }^{6,7,32,53}$ and 
leaves the geometry ( $\lambda, P$, and $R$ ) unchanged, which results in a change of $\Psi$ via varying $T$. Thus, experimentally, the transition at $\Psi^{*}(\lambda)$ corresponds to a de facto irreversible transition from separation to stiction of the colloid as a function of temperature.

Moreover, from Fig. 10 one can infer that for both $d=3$ and $d=4$ there is a $\lambda_{0}$ such that, for $1 \geq \lambda>\lambda_{0}, K_{\mathrm{p}}$ has no zero for any choice of $\Psi$ (i.e., there is no solution $\Theta_{0}$ ) and the critical Casimir force is attractive at all distances. Within the DA, $\lambda_{0}=\Delta_{(+,-)} /\left(\Delta_{(+,-)}-\Delta_{(-,-)}\right)$[see also Eq. (37)], which renders the values $\lambda_{0}=0.80$ in $d=4^{35}$ and $\lambda_{0} \simeq 0.88$ in $d=3 \frac{52}{}$. In addition, from Fig. 10 one can infer that for $\lambda_{0}>\lambda>\lambda_{1} \simeq 0.5$ and $\Psi \lesssim 1, \Theta_{0, s}$ effectively does no longer depend on $\Psi$ but solely on $\lambda$. Accordingly, the distance $D_{0, s} \propto \xi_{+}$at which the colloid stably levitates can be tuned by temperature upon approaching criticality. However, for $\lambda<\lambda_{1} \simeq 0.5, \Theta_{0, s}$ diverges at $\Psi=\Psi_{0}(\lambda)<\Psi^{*}(\lambda)$ such that for $\Psi_{0}(\lambda)<\Psi<\Psi^{*}(\lambda)$ the colloid exhibits critical Casimir levitation at a local minimum of the potential, whereas within this range of $\lambda$ values for $\Psi<\Psi_{0}(\lambda)$ the critical Casimir potential has only a local (positive) maximum at $D_{0, u}$; it is repulsive for $D>D_{0, u}$ and therefore for large values of $D$ (i.e., $\Theta \gg 1$ and $\Pi \ll 1$ ) it approaches zero from positive values. This qualitative change in the behavior of the critical Casimir potential occurs at $\lambda=\lambda_{1}$. The value of $\lambda_{1}$ is close to 0.5 because the repulsive and attractive forces for $(+,-)$ and $(-,-) \mathrm{BC}$, respectively, have similar strengths but opposite signs for $\Theta \gg 1$, i.e., $k_{(+,-)}(\Theta \gg 1) \simeq-k_{(-,-)}(\Theta \gg 1)$ for both $d=3$ and $d=4$ [see Eq. (3), where $\left|A_{-} / A_{+}\right| \simeq 1.2$ in $d=3^{7}$ and $\left|A_{-} / A_{+}\right|=1$ in $\left.d=4^{35}\right]$. Accordingly, depending on $\lambda$ being larger or smaller than $\lambda_{1} \simeq 0.5$, the area covered by one of the two BC prevails and the resulting force is asymptotically (i.e., $\Theta \gg 1$ ) attractive or repulsive, respectively [see the remark at the end of Sec. VIA] and Eqs. (37) and A29]. Taking into account the slight difference in the strength of the asymptotic forces for $(+,-)$ and $(-,-) \mathrm{BC}$ one finds $\lambda_{1}=\left(1-A_{+} / A_{-}\right)^{-1}$ which renders $\lambda_{1}=1 / 2$ in $d=4$ and $\lambda_{1} \simeq 0.545$ in $d=3$. The asymptotic behavior of the force at large distances can be inferred from the asymptotic behavior of $K_{\mathrm{p}}\left(\lambda, \Pi=\Psi \Theta^{-1 / 2}, \Xi=0, \Theta \gg 1, \Delta \rightarrow 0\right) \simeq$ $\mathscr{A}(\Psi, \lambda) \Theta^{d-1} e^{-\Theta}$, which can be obtained from Eqs. 35, (33), (A29), 20), and (3) . Accordingly, the value $\Psi_{0}(\lambda)$ at which $\Theta_{0, s}$ diverges is characterized by the fact that $\mathscr{A}(\Psi \lessgtr$ $\left.\Psi_{0}(\lambda), \lambda\right) \gtrless 0$ so that the force approaches zero from above or from below depending on having $\Psi<\Psi_{0}(\lambda)$ or $\Psi>\Psi_{0}(\lambda)$, respectively. The condition $\mathscr{A}\left(\Psi_{0}(\lambda), \lambda\right)=0$ yields the following implicit equation for $\Psi_{0}(\lambda)$ :

$$
2 \lambda_{1}=\sum_{n=-\infty}^{\infty} \operatorname{erf}\left\{\frac{\Psi_{0}(\lambda)}{\sqrt{2}}\left(n+\frac{\lambda}{2}\right)\right\}-\operatorname{erf}\left\{\frac{\Psi_{0}(\lambda)}{\sqrt{2}}\left(n-\frac{\lambda}{2}\right)\right\} \text {. }
$$

For $\lambda \ll 1$ the sum on the rhs of Eq. (44) can be approximated by the term $n=0$ alone and one finds $\Psi_{0}(\lambda \ll 1) \simeq$ $2^{3 / 2} \lambda^{-1} \operatorname{erf}^{-1}\left(\lambda_{1}\right)$, where $\operatorname{erf}^{-1}$ is the inverse error function, which yields the relations $\Psi_{0}(\lambda \ll 1) \simeq 1.49 / \lambda$ for $d=3$ and $\Psi_{0}(\lambda \ll 1) \simeq 1.35 / \lambda$ for $d=4$. On the other hand, in the marginal case one expects $\Psi_{0}\left(\lambda=\lambda_{1}\right)=0$. However, as argued above, at the critical point $(\Theta=0)$ the colloid does not exhibit stable levitation for any geometrical configuration; this is in accordance with Fig. 10 because for $T \rightarrow T_{c}$, the levitation minimum of the potential moves to large $D$ $\left(D_{0, s}=\Theta_{0, s} \xi_{+} \rightarrow \infty\right)$ and disappears at $T=T_{c}$.
In summary, as function of $\lambda$ there are three distinct levitation regimes:

(i) $\lambda>\lambda_{0}$ with $\lambda_{0}(d=3) \simeq 0.88$ and $\lambda_{0}(d=4)=4 / 5$ : There is no levitation and the critical Casimir force is attractive at all distances for any temperature.

(ii) $\lambda_{0}>\lambda>\lambda_{1}$ with $\lambda_{1}(d=3) \simeq 0.545$ and $\lambda_{1}(d=4)=$ 1/2: Sufficiently close to $T_{c}$, i.e., for $\Psi=P / \sqrt{R \xi_{+}}<$ $\Psi^{*}(\lambda)$ there is a local critical Casimir levitation minimum. Upon approaching $T_{c}$ its position $D_{0, s}=\Theta_{0, s} \xi_{+}$, with $\Theta_{0, s}\left(\xi_{+} \rightarrow \infty\right)$ finite, moves to macroscopic values proportional to the bulk correlation length.

(iii) $\lambda_{1}>\lambda$ : As in (ii) there is a local critical Casimir levitation minimum sufficiently close to $T_{c}$, i.e., for $\Psi<\Psi^{*}(\lambda)$. In general the onset of its appearance occurs further away from $T_{c}$ upon lowering $\lambda$. Upon approaching $T_{c}$ the position $D_{0, s}$ of this minimum diverges at a distinct nonzero reduced temperature given by $\Psi_{0}(\lambda)$, i.e., at $\xi_{+}=P^{2} /\left[R \Psi_{0}^{2}(\lambda)\right]: D_{0, s}=\Theta_{0, s} \xi_{+}$ with $\Theta_{0, s}\left(\Psi \searrow \Psi_{0}(\lambda)\right) \rightarrow \infty$.

We note that, according to Figs. 6, 7, 8 and 9, we expect that for $\Pi \lesssim 2$ and $\Theta \lesssim 4$ and for $\Pi \lesssim 0.5$ and $\Theta \gtrsim 4$, the DA does not provide a quantitatively reliable description of the actual behavior of $K_{\mathrm{p}}$ and therefore of $F_{\mathrm{p}}$; thus, for values of $\Psi \lesssim 2 \sqrt{\Theta_{0}}$ for $\Theta_{0} \lesssim 4$, and $\Psi \lesssim 0.5 \sqrt{\Theta_{0}}$ for $\Theta_{0} \gtrsim 4$, we expect quantitative discrepancies between the actual behavior and the one predicted by the DA shown in Fig. 10 Nonetheless our results demonstrate that the geometric arrangement of the chemical patterns allows one to design the normal critical Casimir force over a wide range.

Figures 11(a) and (b) show the critical Casimir potential $\Phi_{\mathrm{p}}$ as a function of $D$ in $d=3$ within the DA based on Monte Carlo simulation data for the film geometry 52 for a variety of specifically chosen values of the parameters $P, L_{1}, R$, and $\xi$. The choice of these values is motivated by the typical experimental parameters which characterize recent investigations of the critical Casimir force acting on colloids immersed in binary liquid mixtures $6,7,32,53$. In particular, concerning the colloid radius we focus on the data of Ref. 53, corresponding to $R=1.35 \mu \mathrm{m}$, while for the pattern we have chosen a periodicity $P=1 \mu \mathrm{m}$ with $\lambda=0.4$ (i.e., $L_{1}=400 \mathrm{~nm}$ and $L_{2}=600 \mathrm{~nm}$ ) [Fig. 11 a)], or $P=0.4 \mu \mathrm{m}$ with $\lambda=0.65$ (i.e., $L_{1}=260 \mathrm{~nm}$ and $L_{2}=140 \mathrm{~nm}$ ) [Fig. 111(b)]. A chemically patterned substrate with these characteristics appears to be realizable with presently available preparation techniques $32,54,55$. [We note that $\Phi_{\mathrm{p}}$ as shown in Fig. 11(a) and (b) is expected to describe the actual interaction potential in the scaling regime characterized by values of $D$ and $\xi_{+}$much larger than microscopic length scales (such as $\xi_{0}^{+} \simeq 0.2 \mathrm{~nm}^{6,7}$ ) so that this prediction for $\Phi_{\mathrm{p}}$ is valid only for $D, \xi_{+} \gtrsim 5 \mathrm{~nm}$.] With this choice of parameters we have calculated $\Phi_{\mathrm{p}}$ for various values of $\xi_{+}$within an experimentally accessible range $\mathrm{e}^{6,7,32,53}$. From Figs. 11 a) and 11 (b) one can infer that for small values of $\xi_{+}$(corresponding to large values of $\Psi>\Psi^{*}(\lambda)$ ) the critical Casimir potential is always attractive with a monotonic dependence on $D$ [see also Fig. 10]. Upon approaching criticality, i.e., for increasing values of $\xi_{+}$and decreasing values of $\Psi<\Psi^{*}(\lambda)$, a local maximum and a local minimum of the potential develop, so that for very small as well as for large 

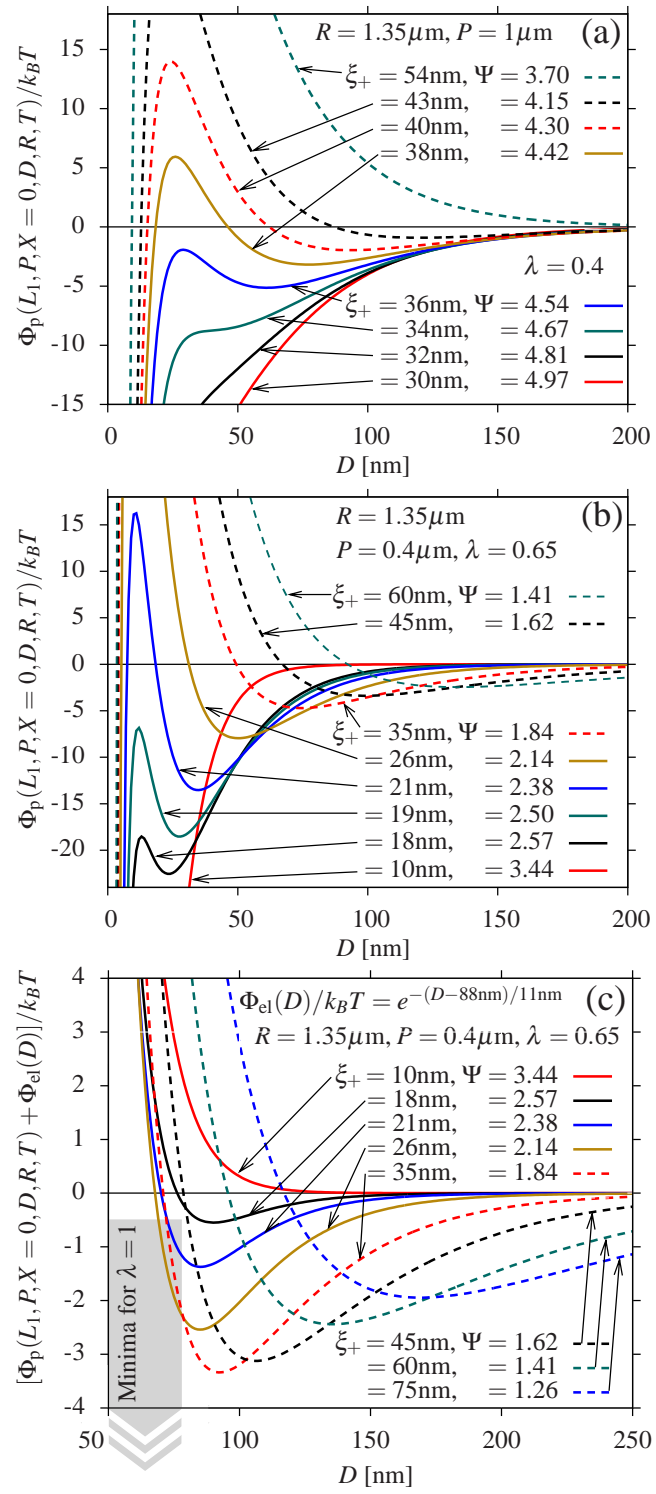

FIG. 11. Critical Casimir potential $\Phi_{\mathrm{p}}$ [Eq. 32] ] in $d=3$ of a colloid of radius $R=1.35 \mu \mathrm{m}$ close to a periodically patterned substrate as a function of $D$ and for various values of $\xi_{+}$for $P=1 \mu \mathrm{m}$ with $\lambda=0.4$ in (a) and $P=0.4 \mu \mathrm{m}$ with $\lambda=0.65$ in (b) and (c). The values of $P, \lambda$, and $\xi_{+}$are chosen as to be experimentally accessible in a colloidal suspension exhibiting critical Casimir forces $6,7,32,53$. The critical Casimir potential for the colloid close to a patterned substrate may exhibit - depending on the value of $\xi_{+}$, and, thus, on the temperature - a local minimum corresponding to stable levitation. In (c) an electrostatic potential $\Phi_{\text {el }}$ [Eq. 45] ] is added to $\Phi_{\mathrm{p}}$, which refers to actual experimental data ${ }^{53}$. The shaded area indicates the ranges of the positions and the depths of the local minima of the total potential occurring if the substrate is laterally homogeneous and purely attractive, i.e., for $\lambda=1((-,-)$ BC) for the range $14 \mathrm{~nm}$ $<\xi_{+}<75 \mathrm{~nm}$ leading to potential depths between $0.5 k_{B} T$ and $70 k_{B} T$ (indicated by the shaded arrow); for $\lambda=1$ the preferred colloid position is dictated by the electrostatic repulsion and restricted to the range of $50 \mathrm{~nm}$ to $75 \mathrm{~nm}$, whereas the colloid position $D_{0, s}=\Theta_{0, s} \xi_{+}$ due to critical Casimir levitation can be much larger and tuned by temperature. Moreover, whereas for $\lambda=1$ and upon approaching $T_{c}$ the minima monotonically become deeper, the levitation minima first deepen and move to smaller values of $D$ followed by a decrease of the depth, by becoming more shallow, and moving to larger values of $D$. Reducing the range and strength of the electrostatic repulsion by adding salt to the solvent is expected to provide access to even more details of the critical Casimir levitation potential $\Phi_{\mathrm{p}}$ shown in (b).
$D$ the colloid is attracted to the patterned substrate, whereas within an intermediate range of values for $D$ it is repelled from it [see also Fig. 10]. Thus, the colloid stably levitates at a distance $D_{0, s}$ corresponding to a local minimum of the potential. The depth of this minimum ranges between a few $k_{B} T$ [Fig. 11(a)] up to several $k_{B} T$ [Fig. 11(b)]. Upon increasing $\xi_{+}, D_{0, s}$ increases as well, i.e., the colloid position is shifted away from the patterned substrate with the potential minimum becoming more shallow. In Fig. 11 (a) $\lambda=0.4$ and we find $\Psi^{*}(\lambda=0.4) \simeq 4.65$ and $\Psi_{0}(\lambda=0.4) \simeq 3.71$ [see Fig. 10(b)] so that for $\Psi<\Psi_{0}(\lambda=0.4)$, i.e., for $\xi_{+} \gtrsim 53.5 \mathrm{~nm}$ [Fig. [11 a)] the colloid does not exhibit stable levitation and the critical Casimir potential has a local maximum only. The levitation minimum moves to macroscopic values of $D$ upon approaching the temperature corresponding to $\xi_{+} \simeq 53.5 \mathrm{~nm}$. In Fig. 11 b) $\lambda=0.65$ and one has $\Psi^{*}(\lambda=0.65) \simeq 2.63$; here $\Theta_{0, s}$ remains finite for $\Psi \rightarrow 0$ in contrast to the case $\lambda<0.545$ [Fig. 10(b)]. Thus, within the DA, for the case shown in Fig. 11(b) stable levitation of the colloid is preserved for all finite values of $\xi_{+}>P^{2} /\left[R\left(\Psi^{*}(\lambda=0.65)\right)^{2}\right] \simeq 17 \mathrm{~nm}$. In this case upon approaching $T_{c}$ the levitation minimum moves to macroscopic values of $D$ proportional to the bulk correlation length $\xi_{+}$.

The discussion above focuses on the position of mechanical equilibrium of the colloid, corresponding to the point at which the forces acting on the particle vanish and the associated potential $\Phi$ has a local minimum $\Phi_{\min }$. However, due to the thermal fluctuations of the surrounding near-critical fluid at temperature $T$, the colloid undergoes a Brownian diffusion which allows it to explore randomly such regions in space where the potential $\Phi$ is typically larger than $\Phi_{\min }$ for at most few $k_{B} T$. As a result, a position of mechanical equilibrium is stable against the effect of thermal fluctuations only if the potential depth of the minimum is larger than few $k_{B} T$. In particular, if the potential barrier $\Phi\left(L_{1}, P, 0, D=\right.$ $\left.D_{0, u}, R, T\right)-\Phi\left(L_{1}, P, 0, D=D_{0, s}, R, T\right)$, which separates the position of the local minimum at distance $D=D_{0, s}$ (levitation) from the global one at $D=0$ (stiction), is not sufficiently large [see, e.g., the curves corresponding to $\xi_{+}=36 \mathrm{~nm}$ in Fig. 11 (a) or corresponding to $\xi_{+} \lesssim 18 \mathrm{~nm}$ in Fig. 11(b)], a de facto irreversible transition from levitation to stiction may occur as a consequence of thermal fluctuations.

In Fig. 11(c) we show the resulting total potential of the forces acting on the colloid in the presence of an additional electrostatic repulsion which is experimentally practically unavoidable, in order to study its effect on critical Casimir levitation. We assume that the electrostatic repulsion is laterally homogeneous and that it can be simply added to the critical Casimir potential 1,31,53 [see also Sec. VIII below]. Concerning the spatial dependence of the electrostatic repulsion we consider the one of Ref. 53, which corresponds to a colloid of radius $R=1.35 \mu \mathrm{m}$ immersed in a near-critical water-lutidine mixture and close to a substrate exhibiting critical adsorption of water or lutidine 53 :

$$
\Phi_{\mathrm{el}}(D) / k_{B} T=\exp \left\{-\kappa\left(D-D_{0}\right)\right\}
$$

where $D_{0}=88 \mathrm{~nm}$ and $\kappa^{-1}=11 \mathrm{~nm}^{53}$. (Formally, $\Phi_{\mathrm{el}}$ in Eq. (45) is finite for $D \rightarrow 0$, and thus $\Phi_{\mathrm{p}}+\Phi_{\mathrm{el}}$ is negative for $D \lesssim 2 \mathrm{~nm}$ and has a global minimum at $D=0$ because $\Phi_{\mathrm{p}} \rightarrow-\infty$ for $D \rightarrow 0$. However, Eq. (45) is actually the asymptotic form of the electrostatic interaction which is valid 
for distances larger than the electrostatic screening length, i.e., $D \gg \kappa^{-1}$. The corresponding total potential $\Phi_{\mathrm{p}}+\Phi_{\mathrm{el}}$ is therefore not accurate for small values of $D$ and is reported in Fig. 11 (c) for $D>50 \mathrm{~nm}$ only.) As in Fig. 11 (b) we choose $P=0.4 \mu \mathrm{m}, \lambda=0.65$, and experimentally accessible values of $\xi_{+}$. Figure 11 (c) provides a realistic comparison of the critical Casimir potential with other forces as they typically occur in actual experimental systems. One can infer from the graph reported in Fig. 11 (c) that for this choice of parameters the critical Casimir levitation exhibited by the colloid is rather pronounced even in the presence of electrostatic interaction. Far from the critical point $\left(\xi_{+}=10 \mathrm{~nm}\right)$ the interaction of the colloid with the substrate is completely dominated by electrostatic repulsion. Upon approaching criticality $\left(10 \mathrm{~nm} \lesssim \xi_{+} \lesssim 35 \mathrm{~nm}\right)$ a minimum in the total potential develops and becomes deeper due to the increasing critical Casimir attraction working against the electrostatic repulsion. For this latter range of values of $\xi_{+}$the local minimum of the critical Casimir potential corresponding to levitation is located at distances $D_{0, s} \lesssim 60 \mathrm{~nm}$ at which the electrostatic repulsion still strongly contributes to the resulting total potential [see Fig. [11(c)]. Closer to the critical point $\left(\xi_{+} \gtrsim 45 \mathrm{~nm}\right)$ the levitation minimum of the critical Casimir potential occurs at distances $D_{0, s} \gtrsim 100 \mathrm{~nm}$ [see Fig. 11 b)] at which the electrostatic force acting on the colloid is weak. Thus, here the critical Casimir effect dominates and the position of the minimum of the total potential increases with increasing values of $\xi_{+}$, which allows for measurements of the critical Casimir potential for distances at which the precise form of $\Phi_{\mathrm{el}}$ is not important. Moreover, the depth of the minimum decreases upon approaching criticality and the minimum becomes more shallow. This behavior of the levitation minimum is distinct from the critical Casimir effect acting on a colloid close to a homogeneous substrate: a local minimum also occurs in the latter case if the critical Casimir force is purely attractive $(\lambda=1,(-,-)$ BC) and works against the electrostatic repulsion ${ }^{6.7}$, due to the competition of different forces with opposite sign. (We note that the critical Casimir levitation described above emerges from the critical Casimir force alone, i.e., it is a feature of a single force contribution.) However, in this homogeneous case the preferred colloid position $D_{0,(-,-)}$ depends crucially on the form of the electrostatic interaction and is almost constant $\left(50 \mathrm{~nm}<D_{0,(-,-)}<75 \mathrm{~nm}\right)$. Moreover, the depths of these latter minima monotonically increase as a function of of $\xi_{+}$and become much larger than those shown in Fig. 11 (c) (see, e.g., Fig. 2(a) and Fig. 2(c) in Ref. 6 and Fig. 3 in Ref. 53). In Fig.11(c) this is indicated by the shaded area and the shaded arrow, which corresponds to the area of the graph within which minima of the total potential in the homogeneous case $\lambda=1$ occur for $14 \mathrm{~nm}<\xi_{+}<75 \mathrm{~nm}$ corresponding to potential depths of $0.5 k_{B} T$ up to $70 k_{B} T$. On the other hand, the colloid position $D_{0, s}$ due to critical Casimir levitation can be much larger, can reach values of several $\xi_{+}$, and can be tuned by temperature according to $D_{0, s}=\Theta_{0, s} \xi_{+}$. In conclusion, the examples presented in Fig. 11 strongly suggest that the critical Casimir levitation of a colloid close to a patterned substrate is experimentally accessible.

By patterning the substrate, one introduces an additional (lateral) length scale into the system, which, according to our results presented above, can finally lead to stable levitation. Introducing an additional length scale along the nor- mal direction by stacking different materials on top of each other may lead to levitation due to quantum-electrodynamic Casimir forces ${ }^{56}$. The behavior of the stable levitation distance shows a bifurcation and irreversible transitions from separation to stiction ${ }^{56}$ similarly to the ones described above [see Fig. 10]. In that context great importance has been given to the temperature dependence of the position $D_{0, s}$ of stable quantum Casimir levitation ${ }^{56}$, which is quantified by the value of $\frac{d}{d T} D_{0, s}$. In the critical Casimir case presented here, for an estimate of $\frac{d}{d T} D_{0, s}$ we pick as an example the stable levitation positions for $\xi_{+}=18 \mathrm{~nm}$ and $\xi_{+}=60 \mathrm{~nm}$ as reported in Fig.11 (a different choice would lead to similar results). The results reported in Fig. 11 correspond to the experimentally relevant water-lutidine mixture with $\xi_{0}^{+}=0.2 \mathrm{~nm}$ and $T_{c} \simeq 307 \mathrm{~K}^{6,7,53}$. Therefore, according to $\xi_{+} / \xi_{0}^{+}=\left|\left(T-T_{c}\right) / T_{c}\right|^{-v}$, the difference in temperature required to move from $\xi_{+}=18 \mathrm{~nm}$ to $\xi_{+}=60 \mathrm{~nm}$ is $\Delta T \simeq 0.2 \mathrm{~K}$. Thus we find $\frac{d}{d T} D_{0, s} \simeq 560 \mathrm{~nm} \mathrm{~K}^{-1}$ for the average temperature dependence of critical Casimir levitation [Fig. [11 b)], and $\frac{d}{d T} D_{0, s} \simeq 230 \mathrm{~nm} \mathrm{~K}^{-1}$ by additionally taking electrostatics into account [Fig. 11) (c)]. We note that in the present critical case $\frac{d}{d T} D_{0, s}$ can become arbitrarily large at temperatures corresponding to the transition from separation to stiction and the emergence of the local minimum and the local maximum of the critical Casimir potential [see Fig. 10 and the curves for $\xi_{+}=34 \mathrm{~nm}$ and $\xi_{+}=36 \mathrm{~nm}$ in Fig. [11(a)]. This shows that the critical Casimir levitation is strongly temperature dependent, even near room temperature, with the variation of stable separation $\frac{d}{d T} D_{0, s}$ being two orders of magnitude larger than the one predicted for the quantumelectrodynamic Casimir effect in Ref. 56. In general the colloid will not only be exposed to the critical Casimir force and to an electrostatic force but also to gravity and to laser tweezers, which generate a linearly increasing potential contribution. This attractive contribution tends to reduce the potential barriers shown in Fig. 11 and can eliminate small barriers altogether. Thus these external forces can be used to switch levitation on and off (compare a similar discussion related to the quantum-electrodynamic Casimir levitation in Ref. 56).

\section{CYLINDER}

Currently, there is an increasing experimental interest in elongated colloidal particles which have a typical diameter of up to several $100 \mathrm{~nm}$ and a much larger length (see, e.g., Refs. 30 and 57 and references therein). These types of colloids resemble cylinders rather than spheres. The description of their behavior in confined critical solvents calls for a natural extension of the studies presented in Secs. [IIIVI] Hence, in the present section we consider the case of a $3 d$ cylinder with (-) BC which is adjacent and parallel aligned to a periodically chemically patterned substrate consisting of alternating $(-)$ and $(+)$ stripes as the ones discussed in Sec. VI Accordingly, the axis of rotational invariance of the cylinder is perpendicular to both the $x$ direction [Fig. 1] and the direction normal to the substrate, and it is parallel to the direction of spatial translational invariance of the chemical stripes forming the pattern. As compared with the case of the sphere the analysis for the cylinder is technically simpler because the system as a whole is invariant along all directions but two, the lateral one, $x$, and the one normal to the substrate. (For 
the sphere its finite extension in the second lateral direction, which is normal to the $x$-axis, matters and thus leads to a basically three-dimensional problem. Accordingly, here we do not consider short cylinders, for which this finite length matters, too.) This reduction of the number of relevant dimensions allows us to perform numerical calculations of adequate precision for a range of various pattern geometries which is wider than in the case of the sphere. (Here, we do not consider a cylindrical colloid which is not perfectly aligned with the pattern and which would, therefore, experience a critical Casimir torque ${ }^{30}$.) Even though the expressions derived in Appendix $D$ can be used to study the case of a cylinder having its axis laterally displaced by an arbitrary amount $X$ from the chemical step, our numerical calculations for the case of a chemical stripe address only the case $X=0$. This corresponds to a lateral position of the symmetry axis of the cylinder which coincides with the center of an attractive $(-)$ stripe.

In Appendix $\mathrm{D}$ we briefly derive the scaling behavior of the normal critical Casimir force acting on the cylinder and compare it with the case of a sphere. Then, we adapt the Derjaguin approximation appropriate for the geometry of the cylinder. On this basis, we have calculated the scaling function of the normal critical Casimir force acting on the cylinder in $d=3$ and $d=4$ on the basis of the Monte Carlo simulation data for the film geometry 52 and of the analytic MFT expression for the critical Casimir force for the film geometry 35 , respectively. In addition, within the same approach as the one of Sec. III we have calculated numerically the MFT scaling functions corresponding to $\Delta \neq 0$, in order to assess the performance of the DA.

Here we focus on the comparison between the DA appropriate for the cylinder and the full numerical MFT data for the scaling function $K_{\mathrm{p}}^{\mathrm{cyl}}(\lambda, \Pi, \Xi=0, \Theta, \Delta)$ which characterizes the normal critical Casimir force in the presence of a periodically patterned substrate; $\lambda, \Pi, \Xi, \Theta$, and $\Delta$ are defined as in the case of the sphere [see Sec. VI and Appendix $\mathrm{D}$. Figure 12 shows the scaling function of the normal critical Casimir force acting on a cylinder as a function of $\Theta$ as obtained from the DA $(\Delta \rightarrow 0)$ in $d=4$ and from the full numerical MFT calculations for $\Delta=1 / 3$. Besides the quantitative differences in the scaling function as a function of $\Theta$, the qualitative features of the behavior of the force acting on a cylinder, which is reported in Fig. 12 for various values of $\lambda$, are similar to the ones for the sphere [compare Fig. 9]. For $\Pi=1.92$ [Fig. 12(a)] the DA describes the actual behavior of the critical Casimir force rather well, in particular for $\Theta \gtrsim 2$, even for most values of $\lambda$. As in Fig. 9, for a certain range of values of $\lambda$ the normal critical Casimir force changes sign at $\Theta_{0}^{\text {cyl }}(\Pi, \lambda, \Xi=0, \Delta)$. On the other hand for small periodicities ( $\Pi=0.29$ in Fig. 12 b) ) the DA in $d=4$ fails to describe quantitatively the actual behavior of the force as obtained from the full numerical MFT calculations. These strong deviations from the DA [Fig. 12(b)] indicate the relevance of effects caused by the actual non-additivity of critical Casimir forces.

For $\lambda \gtrsim 0.6$ the scaling function $K_{\mathrm{p}}^{\mathrm{cyl}}$ of the normal critical Casimir force obtained numerically and represented by symbols in Fig. 12 b) is very close (much closer than within the DA) to the one corresponding to the homogeneous case with $(-,-) \mathrm{BC}$ (corresponding to $\lambda=1$ ) and does not show a change of sign. This means that, even if the substrate is
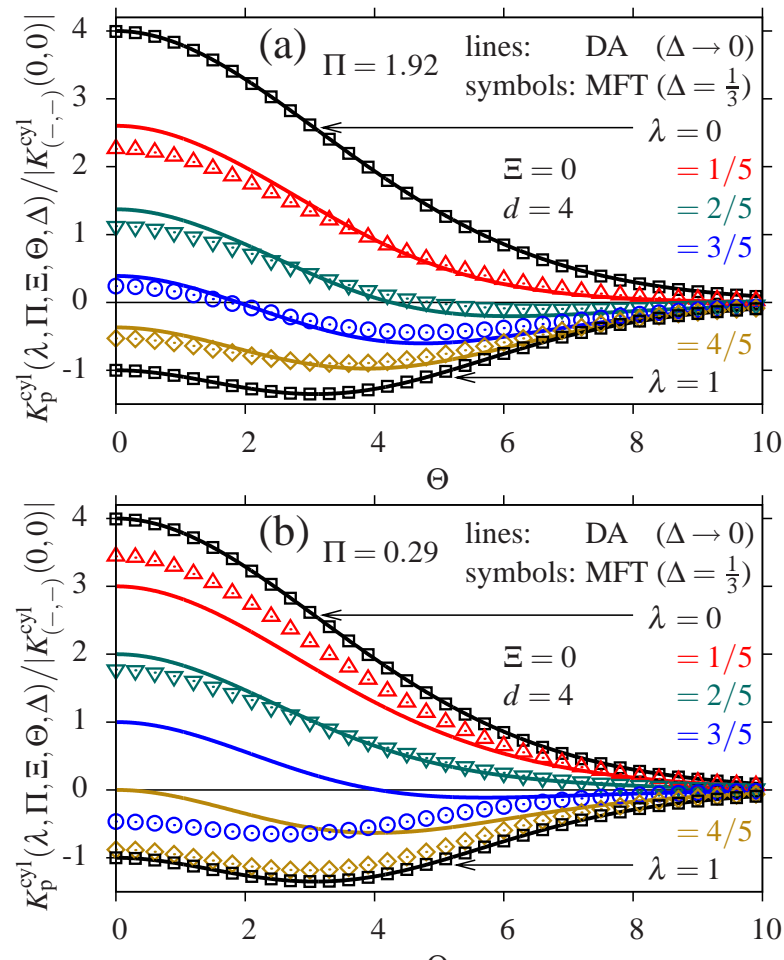

$\Theta$

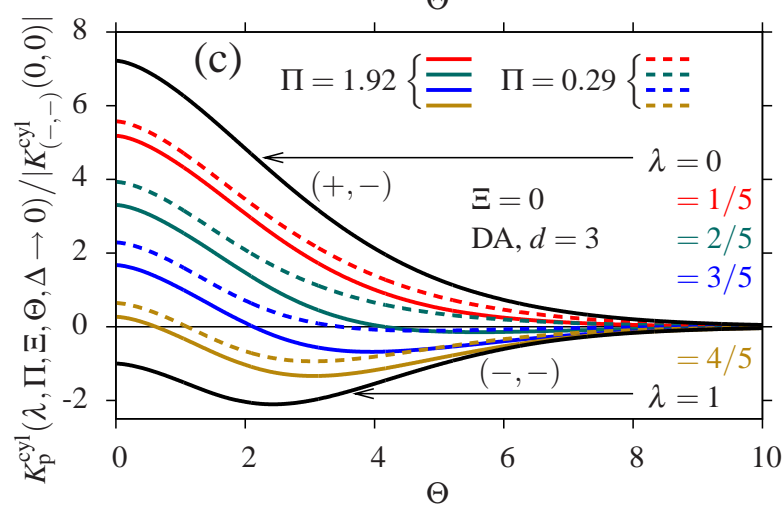

FIG. 12. Normalized scaling function $K_{\mathrm{p}}^{\mathrm{cyl}}$ [see Appendix D including expressions for $\left.K_{(-,-)}^{\mathrm{cyl}}(0,0)\right]$ of the normal critical Casimir force acting on a cylindrical colloid close to and parallel to a periodically patterned substrate. The cylinder axis is aligned with the striped pattern and positioned above the center of a (-) stripe which has the same adsorption preference as the cylinder (analogous to Fig.9 for a spherical colloid). In (a) for $\Pi=1.92$ the appropriate DA describes the actual MFT data rather well, and for $0.3 \lesssim \lambda \lesssim 0.7$ there is a change of sign of the force. In (b), instead, apart from the limiting homogeneous cases $\lambda=0$ and $\lambda=1$, for $\Pi=0.29$ the DA fails to describe quantitatively the actual behavior [see the main text]. In (c) $K_{\mathrm{p}}^{\mathrm{cyl}}$ is shown for $d=3$ within the DA based on the Monte Carlo simulation data for the film geometry 52 for the two cases $\Pi=0.29$ and $\Pi=1.92$. We expect that also in $d=3$ the DA for $\Pi=0.29$ is not quantitatively reliable.

not homogeneous but chemically patterned - but such that the larger part of the surface still corresponds to (-) BC, i.e., $\lambda \gtrsim 0.5-$ the resulting critical Casimir force acting on the colloid with (-) BC resembles the behavior for laterally homogeneous $(-,-)$ BC. This can be understood in terms of the fixed point Hamiltonian in Eq. (4) which penalizes spatial variations of the order parameter at short scales. Thus 
the system tries to smooth out spatial inhomogeneities of the order parameter profile, biased by the preference of the colloidal particle. If the pattern is very finely structured, i.e., $\Pi=\left(L_{1}+L_{2}\right) / \sqrt{R D} \ll 1$, regions with a positive order parameter close to the narrow $(+)$ stripes $\left(\lambda \simeq 1^{-}\right.$, i.e., $\left.L_{2} \ll L_{1}\right)$ extent only very little into the direction normal to the substrate and the resulting order parameter profile at a distance from the substrate remains negative only ${ }^{58}$, so that the force resembles the one corresponding to the homogeneous case. (Note that within the DA, the corresponding order parameter profile would simply consist of a patchwork of the order parameter profiles corresponding to the film geometry, with no smoothing taking place at the edges of the various spatial regions.) Similarly, but in a weaker manner due to the opposite order parameter preference at the colloid, the curves in Fig. 12 b) for $\lambda \lesssim 0.5$ approach the corresponding homogeneous one for the case $(+,-)$ (i.e., $\lambda=0)$. Thus, the fact that both in Fig. 12 (a) and Fig. 12 (b) the curves for $\lambda=1 / 5$ are less close to their limiting ones for $\lambda=0$ than the curves for $\lambda=4 / 5$ are close to the ones for $\lambda=1-$ although the portions of the minority part of the surface are the same - is due to the fact that an order parameter profile with $(+,-)$ boundary conditions is energetically less preferred than the one with $(-,-)$ boundary conditions because in the $(+,-)$ case an interface emerges between the two phases. For broad stripes, i.e., in contrast to the case $\Pi \rightarrow 0$, the energy costs for a similar behavior are seemingly larger: the full numerical MFT data for $\lambda=1 / 5$ and $\lambda=4 / 5$ are less close to the corresponding limiting homogeneous cases $\lambda=0$ and $\lambda=1$, respectively, for $\Pi=1.92$ than for $\Pi=0.29$.

Figure 12 (c) shows the scaling function $K_{\mathrm{p}}^{\mathrm{cyl}}$ of the normal critical Casimir force for $d=3$ within the DA as obtained by using Monte Carlo simulation data for the film geometry ${ }^{52}$. One can infer from Fig. 12 (c) that the qualitative features of the MFT scaling function as described above, such as the change of sign, are carried over to $d=3$.

As discussed in the previous section, the vanishing of the normal critical Casimir force corresponds to a stable levitation of the colloid at a distance $D_{0}$ from the substrate only if $\left.\partial_{D} F_{\mathrm{p}}^{\mathrm{cyl}}\right|_{D=D_{0}}<0$. Within the DA and at the laterally stable position $\Xi=0$ the sign of $\partial_{D} F_{\mathrm{p}}^{\mathrm{cyl}}$ is given by Eq. (43) with $K_{\mathrm{p}}$ replaced by $K_{\mathrm{p}}^{\mathrm{cyl}}$. The behavior of $\Theta_{0}^{\text {cyl }}$ as a function of $\Psi$ and the demarcation of the regions where levitation is stable against perturbations of $D$ is shown in Fig. 13, where the solid and the dashed lines correspond to stable and unstable levitation, respectively. The behavior for the normal critical Casimir force acting on the cylinder is qualitatively similar to the one for the sphere shown in Fig. 10, Analogously to the case of a sphere discussed in Sec. VIC no stable levitation is found at $T=T_{c}$ or for $\lambda>\lambda_{0}=\Delta_{(+,-)} /\left(\Delta_{(+,-)}-\Delta_{(-,-)}\right)$, where $\lambda_{0}=0.80$ in $d=4$ and $\lambda_{0} \simeq 0.88$ in $d=3$. On the other hand, for $\Theta>0$, and $\lambda<\lambda_{0}$, it is always possible to find values of $P$ and $R$ such that stable levitation of the cylinder occurs at a certain distance from the substrate. The values of $\lambda_{1}$ below which one has a finite value $\Psi_{0}(\lambda)$ at which $\Theta_{0}$ diverges remain the same as for the case of a sphere, i.e., $\lambda_{1}(d=4)=1 / 2$ and $\lambda_{1}(d=3) \simeq 0.545$; also the corresponding values of $\Psi_{0}(\lambda)$ remain the same [see Eq. (44)].

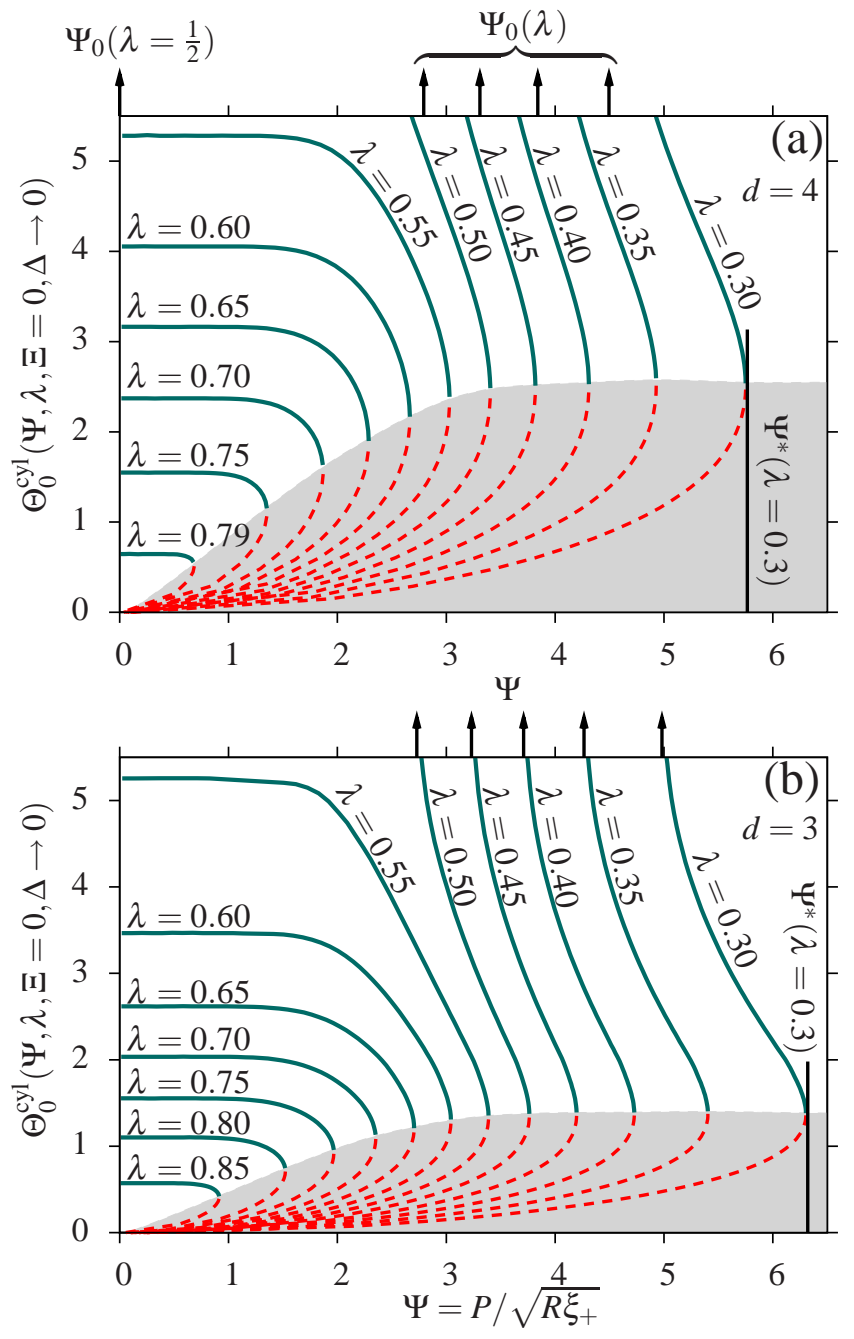

FIG. 13. Values of the scaling variable $\Theta_{0}^{\text {cyl }}$ at which the normal critical Casimir force $K_{\mathrm{p}}^{\mathrm{cyl}}$ acting on a cylinder close to a periodically patterned substrate vanishes as a function of $\Psi=P / \sqrt{R \xi_{+}}$[compare Fig. 10 for the case of a sphere] within the DA. The region indicated by solid lines corresponds to the one in which the levitation of the cylinder at a height $D=D_{0}=\Theta_{0} \xi_{+}$is stable against small perturbations of $D$, wheres in the shaded region indicated by dashed lines there is no such stable levitation although the normal critical Casimir force acting on the colloid vanishes. For $\lambda>\lambda_{0}$ with $\lambda_{0}(d=4)=4 / 5$ and $\lambda_{0}(d=3) \simeq 0.88, \Theta_{0}^{\text {cyl }}$ ceases to exist, i.e., $K_{\mathrm{p}}^{\text {cyl }}$ does not exhibit a zero. For $\lambda<\lambda_{1}$ with $\lambda_{1}(d=4)=1 / 2$ and $\lambda_{1}(d=3) \simeq 0.545, \Theta_{0}^{\text {cyl }}\left(\Psi \searrow \Psi_{0}(\lambda)\right)$ diverges. (The values for $\Psi_{0}(\lambda)$ are indicated by upward arrows.) For any $\lambda<\lambda_{0}, \Theta_{0}^{\text {cyl }}$ exists for $\Psi<\Psi^{*}(\lambda)$. We expect the DA to be quantitatively reliable only for $\Psi / \sqrt{\Theta_{0}} \gtrsim 2$ for $\Theta_{0} \lesssim 4$ and for $\Psi / \sqrt{\Theta_{0}} \gtrsim 0.5$ for $\Theta_{0} \gtrsim 4$.

\section{SUMMARY AND CONCLUSIONS}

We have investigated the universal properties of the normal and lateral critical Casimir forces acting on a spherical or cylindrical colloidal particle close to a chemically structured substrate with laterally varying adsorption preferences for the species of a (near) critical classical binary liquid mixture (at its critical composition) in which the colloid is immersed. Within the Derjaguin approximation (DA) [see Fig. 14] in spatial dimensions $d=3$ and $d=4$ we have derived analytic ex- 
pressions for the corresponding universal scaling functions of the forces and the potentials for general fixed-point boundary conditions (BC) in terms of the scaling function of the critical Casimir force acting on two parallel, homogeneous plates. These expressions are given explicitly analytically at the bulk critical point $T=T_{c}$ and - for symmetry breaking boundary conditions - far away from the critical point. These relations enable one to obtain predictions for actual three-dimensional systems with a sphere-inhomogeneous plate geometry (for which currently computations are not possible) based on the scaling function for the parallel homogeneous plate geometry, for which, e.g., Monte Carlo simulation data in $d=3$ are available. Moreover, results within mean-field theory (MFT, corresponding to $d=4$ ) and symmetry-breaking boundary conditions [Sec. IIB] have been obtained fully numerically and have been compared with the approximate results of the DA, which allows us to explore the limits of validity of the latter. We have studied several relevant situations [see Fig. 1] and our main findings are the following:

1. First, we have studied a spherical colloid immersed in a binary liquid mixture close to a chemically homogeneous substrate which has, compared to the colloid, the same $(-)$ or a different $(+)$ adsorption preference for one of the species of the mixture [Sec. III]]. Close to the bulk critical point at $T=T_{c}$ the critical Casimir force induced by the confinement of the order parameter (e.g., the concentration difference in a binary liquid mixture) can be described in terms of universal scaling functions depending on the surface-to-surface distance $D$ of the colloid from the substrate scaled by the bulk correlation length, $\Theta=\operatorname{sign}\left(\left(T-T_{c}\right) / T_{c}\right) D / \xi_{ \pm}$, and its ratio with the radius of the colloid, $\Delta=D / R$ [Eqs. (5) and (6)]. The scaling functions obtained within the DA [Eqs. (10) and (11)] are valid for $\Delta \rightarrow 0$. From the comparison with the full numerical MFT results [Fig.2] we find that in $d=4$ the DA describes the actual behavior quite well for $\Delta \lesssim 0.4$. Based on Monte Carlo simulation data for the scaling function of the critical Casimir force between parallel, homogeneous plates and within the DA we have obtained also the scaling function for the critical Casimir force on a spherical colloid close to a homogeneous substrate in $d=3$ [Fig.2].

2. The basic building block of a chemically patterned substrate is a chemical step, which we have studied in Sec. IV Due to the broken translational invariance in one lateral direction $(x)$ the critical Casimir forces and potentials acquire a dependence on the additional scaling variable $\Xi=X / \sqrt{R D}$, which corresponds to the lateral distance $X$ of the center of the spherical colloid from the position of the chemical step along the plane [Eqs. (12), 14), and (21)]. Due to the different boundary conditions on both sides of the chemical step a lateral critical Casimir force emerges, which leads to a laterally varying potential for the colloid. In the limit $\Delta \rightarrow 0$ both the scaling function for the potential and for the lateral critical Casimir force as obtained within the DA are in agreement with the full numerical data [Fig. 3]. We have derived the corresponding scaling functions within the DA also in $d=3$ by using Monte Carlo data for the parallel plate geometry [Fig. 3]. The preceding results have been partly presented in Ref. 31 as well as their suitable comparison with corresponding experimental results ${ }^{32}$, which revealed that the critical Casimir effect is rather sensitive to the geometrical details of the substrate patterns.

3. Section $\nabla$ deals with the critical Casimir forces and the corresponding potential acting on a spherical colloid in front of a single chemical lane of width $2 L$, which additionally depends on a fourth scaling variable $\Lambda=L / \sqrt{R D}$ [Eqs. (25) and (26)]. It turns out that within the DA the scaling functions for the critical Casimir force and the critical Casimir potential across a chemical lane can be expressed in terms of the ones for the chemical step [Eqs. (29) and (30)]. For large values of $\Lambda$ the resulting potential can be described as a suitable superposition of chemical steps, whereas for $\Lambda \lesssim 3$ one has explicitly to account for the finite width of the chemical stripe [Fig. 4]. Comparing the results of the DA with the ones obtained by a full numerical analysis, one finds that the DA describes the actual behavior quite well for $\Delta \lesssim 0.4$, even for small $\Lambda$. Seemingly, in this respect, the nonlinearities inherent in the critical Casimir effect and edge effects do not considerably affect the resulting scaling functions [Fig. 5].

4. On the basis of the results of Sec. V] in Sec. VI we have studied the universal scaling functions of the critical Casimir force and the corresponding potential for a sphere opposite to a periodically patterned substrate with laterally alternating chemical stripes of different adsorption preferences [Sec. VI]. These scaling functions [Eqs. (31) and (32)] depend, besides the scaling variables $\Theta, \Delta$, and $\Xi$, on two additional scaling variables $\Pi=P / \sqrt{R D}$ and $\lambda=L_{1} / P$, which correspond to the period $P=L_{1}+L_{2}$ of the pattern and to the width $L_{1} \leq P$ of the stripes with the same adsorption preference as the colloid. The scaling function for the normal critical Casimir force obtained within the DA can be expressed in terms of the one for the chemical step and describes the actual behavior well for $\Pi \gtrsim 2$ [Eq. (35) and Figs. 6, 7(a) and 9(a)]. However, for $\Pi \rightarrow 0$ [Eq. (37)] the DA fails to capture quantitatively the numerically obtained behavior within MFT, reflecting the importance of nonlinearities and edge effects in this context, which are not accounted for by the DA [Figs. 6, 71a) and 9 b)]. The failure of the DA in the limit $\Pi \rightarrow 0$ can be traced back to the fact that for the film geometry of a patterned wall next to a laterally homogeneous flat wall, additivity of the critical Casimir forces does not hold [Fig. 8].

5. The MFT scaling function of the normal critical Casimir force acting on a colloid close to a periodically patterned substrate shows a remarkable behavior as a function of $\Theta=D / \xi_{+}$. Within a certain range of values of $\Pi$ and $\lambda$ the critical Casimir force vanishes at $\Theta_{0}$ corresponding to a distance $D=D_{0}$ between the colloid and the substrate. We have analyzed the sign of the derivative of the critical Casimir force with respect to $D$ at $D_{0}$, which is negative if for $D<D_{0}=D_{0, s}$ the colloid is repelled from the substrate whereas for 
$D>D_{0}=D_{0, s}$ it is attracted to the substrate [Fig. 10]. This means that in the absence of other forces the colloid can levitate above the substrate at a stable distance which can be tuned by temperature. Stable levitation points are found also in $d=3$, within the DA and on the basis of the Monte Carlo data for the parallel plate geometry [Figs.7(b), 9(c), and 10 (b)]. Our analysis shows that at the critical point $T=T_{c}$ levitation is not possible, whereas off criticality a geometrical configuration leading to stable levitation can always be found. For fixed geometrical parameters, the critical Casimir potential as a function of $D$ changes from a monotonic behavior to a non-monotonic one upon approaching criticality; a local maximum and a local minimum, the latter corresponding to stable levitation, occur [Fig. 11 (a) and (b)]. Experimentally, this corresponds to a de facto irreversible transition from separation to stiction of a colloid and a patterned substrate. The depths of these potential minima can be up to several $k_{B} T$ so that the levitation is stable against Brownian motion of the colloid. The critical Casimir levitation can be rather pronounced and robust even in the presence of electrostatic interactions [Fig. 11(c)]. The levitation height is proportional to the bulk correlation length and thus can be tuned by varying temperature. Depending on the geometric parameter $\lambda$ we have identified two distinct types of temperature dependences of the levitation height $D_{0, s}$. In both cases it exhibits a high temperature sensitivity $\frac{d}{d T} D_{0, s}$ which, for realistic examples at room temperature, is of the order of several $100 \mathrm{~nm} \mathrm{~K}^{-1}$. These results show that the periodic patterning of the substrate enables one to design critical Casimir forces over a wide range of properties.

6. This behavior is also observed for a cylindrical colloid which lies parallel to the substrate such that its axis is aligned with the translationally invariant direction of the stripes [Sec.VII] and Appendix[D]. The main features of the scaling function for the corresponding normal critical Casimir force are similar to the ones for the spherical colloid: the DA describes well the actual behavior as obtained from full numerical MFT calculations for large values of $\Pi$, but fails quantitatively for $\Pi \lesssim 2$ [Fig. 12]. The numerical studies for $\Pi \rightarrow 0$ indicate that a substrate with a very fine pattern, dominated by one of the two $\mathrm{BC}$ as far as the corresponding covered area is concerned, leads to a normal critical Casimir force which resembles the one for a homogeneous substrate characterized by the dominating BC [Fig. 12 b)]. Based on Monte Carlo data for the parallel plate geometry we calculated within the DA the critical Casimir force acting on a cylinder in $d=3$ [Fig. 12](c)]. Above a chemically patterned substrate, also for a cylinder stable levitation is possible for a wide range of parameters [Fig. 13].

Typically, in experiments with a colloidal suspension one has to consider also other forces, such as electrostatics, gravitation, and van der Waals forces which act on the colloidal particles in addition to the critical Casimir forces. The total force is approximately the sum of these contributions ${ }^{59,60}$ [see Fig. 11 (c)]. Upon approaching the critical point in the phase diagram, experiments 6.7 .32 and theory (see, e.g.,
Refs. 7, 31, and 33) highlight the importance and the relevance of the critical Casimir effect in comparison with these other forces.

The lateral critical Casimir forces occurring for patterned substrates as discussed here are highly sensitive to the details of the geometry of the pattern. A detailed comparison with available experimental dat ${ }^{32}$ has to take this into account $t^{31}$. This sensitivity even allows for an independent determination of the geometry of a chemically structured substrate by means of the critical Casimir effect. This is useful in cases in which it is difficult to infer the geometry of the chemical pattern directly ${ }^{31,32}$. Concerning the comparison with experiments for chemically structured substrates, the theoretical predictions for the critical Casimir force are in agreement with the presently available dat $a^{31.32}$, for which the description in terms of independent chemical steps [Sec. IV] turns out to be sufficient ${ }^{31}$. In order to test our specific predictions obtained for narrow single chemical lanes and for periodic chemical stripes, structures on the nanometer scale are needed. Preliminary experimental data in this direction are encouraging 54,55 .

In view of present basic research efforts and potential applications, it is important to study the effect of weak critical adsorption of the fluid at the confining surfaces, corresponding to finite surface fields. Such weak surface fields can be realized by applying suitable surface chemistry and they influence the resulting behavior of the critical Casimir effect strongly 15.53 . Another approach to create an effective reduction of the surface adsorption is to create fine periodic chemical patterns with different (strong) adsorption preferences as discussed here. However, our results for $\Pi \rightarrow 0$ [Figs. 9 (b) and [12 b)] show that a fine patterning of the substrate with alternating boundary conditions does not necessarily lead to an effective reduction of the surface adsorption at short distances because in this range the critical Casimir force for a inhomogeneous adsorption preference resembles the one for a homogeneous substrate corresponding to strong adsorption. On the other hand, at large distances a periodically patterned substrate does lead to an effective BC corresponding to a weak adsorption preference, and for $\lambda=1 / 2$ the surface fields even cancel out, leading to an effective $\mathrm{BC}$ resembling the so-called ordinary $\mathrm{BC}^{33}$. This offers the interesting perspective to study, at least asymptotically, critical Casimir forces with Dirichlet $\mathrm{BC}$ by using classical fluids instead of superfluid quantum fluids $10,17,18,61$.

A patterning on the molecular scale is not captured by the continuous approach pursued here, which gives the universal features of the critical Casimir effect. Nonetheless, a molecular patterning of the substrates may provide another means for an effective reduction of the adsorption of the corresponding fluid at the surface. However, on a molecular scale the patterning is more likely to lead to randomly distributed surface fields which opens a new challenge in the context of critical Casimir forces.

\section{ACKNOWLEDGMENTS}

S. K. and L. H. gratefully acknowledge support by grant HA 2935/4-1 of the Deutsche Forschungsgemeinschaft. A. G. is supported by MIUR within the program "Incentivazione alla mobilità di studiosi stranieri e italiani residenti all'estero". 


\section{Appendix A: Derjaguin approximation for a chemical step}

In this appendix we first calculate within the DA the normal critical Casimir force $F_{\mathrm{S}}(X, D, R, T)$ [Eq. (12)] acting on a spherical colloid of radius $R$ facing a chemical step by using the DA. (We cannot directly calculate the lateral critical Casimir force $F_{\mathrm{S}}{ }^{\|}(X, D, R, T)$ within the DA because for two parallel homogeneous plates such a force vanishes.) In a second step we derive the critical Casimir potential $\Phi_{\mathrm{s}}(X, D, R, T)=\int_{D}^{\infty} \mathrm{d} z F_{\mathrm{S}}(X, z, R, T)$ by integrating this result for the normal critical Casimir force. In a third step the lateral critical Casimir force is obtained as $F_{\mathrm{S}}^{\|}(X, D, R, T)=$ $-\partial_{X} \Phi_{\mathrm{s}}(X, D, R, T)=-\int_{D}^{\infty} \mathrm{d} z \partial_{X} F_{\mathrm{s}}(X, z, R, T)$ [see Sec. IVB].

In the spirit of the DA, the surface of the spherical colloid with $(b) \mathrm{BC}$ is thought of as being made of a pile of (infinitely thin) rings parallel to the opposing substrate and with an area $\mathrm{d} S(\rho)=2 \pi \rho \mathrm{d} \rho$, where $\rho$ is the radius of the ring. Each of these rings is partly facing (in normal direction) the surface with $\left(a_{<}\right) \mathrm{BC}$, with an extension $\mathrm{d} S_{<}(\rho)$, and partly facing the surface with $\left(a_{>}\right) \mathrm{BC}$ on the other side of the chemical step [Fig. 14], with an extension $\mathrm{d} S_{>}(\rho)$, such that $\mathrm{d} S(\rho)=\mathrm{d} S_{<}(\rho)+\mathrm{d} S_{>}(\rho)$. For an assigned $\rho, \mathrm{d} S_{\gtrless}(\rho)$ depend, inter alia, on the lateral position $X$ of the colloid. Using the assumption of additivity of the forces underlying the DA we suppose that the contribution $\mathrm{d} F_{\mathrm{S}}(\rho)$ of the ring to the total critical Casimir force $F_{\mathrm{S}}$ is given by the sum of the critical Casimir forces which would act, in a film, on portions of areas $\mathrm{d} S_{<}$and $\mathrm{d} S_{>}$in the presence of $\left(a_{<}, b\right)$ and $\left(a_{>}, b\right) \mathrm{BC}$, respectively. According to Eq. (1) this leads to the following expression for the force acting on a single ring:

$$
\begin{aligned}
\frac{\mathrm{d} F_{\mathrm{S}}(\rho)}{k_{B} T}=\frac{\mathrm{d} S_{<}(\rho)}{L^{d}(\rho)} k_{\left(a_{<}, b\right)}\left(\operatorname{sign}(t) L(\rho) / \xi_{ \pm}\right) \\
\quad+\frac{\mathrm{d} S_{>}(\rho)}{L^{d}(\rho)} k_{\left(a_{>}, b\right)}\left(\operatorname{sign}(t) L(\rho) / \xi_{ \pm}\right)
\end{aligned}
$$

where $L(\rho)$ is the substrate-ring distance [Fig. 14] as given in Eq. (7), and $k_{\left(a_{\gtrless}, b\right)}$ are the scaling functions of the critical Casimir force in the film geometry with $\left(a_{>}, b\right)$ and $\left(a_{<}, b\right)$ $\mathrm{BC}$, respectively [see Eq. (1)]. This assumption neglects all edge effects along the boundary between the areas $\mathrm{d} S_{>}(\rho)$ and $\mathrm{d} S_{<}(\rho)$, which might actually be relevant in view of the spatial variation of the order parameter profile. It is therefore important to test the validity of this assumption at least in some relevant cases. This is carried out in Sec. IV for $d=4$, i.e., within MFT.

Without loss of generality in the following we assume $X>$ 0 , i.e., that the normal projection of the center of the sphere falls on the part of the substrate with $\left(a_{>}\right)$BC [Figs. 1 and 14]. The results for $X<0$ are obtained by exchanging in the formulas below $a_{<} \leftrightarrow a_{>}$and $X \leftrightarrow-X$. Taking into account that $\mathrm{d} S(\rho)=\mathrm{d} S_{<}(\rho)+\mathrm{d} S_{>}(\rho)$ one can rewrite Eq. (A1) as

$$
\begin{aligned}
\frac{\mathrm{d} F_{\mathrm{S}}(\rho)}{k_{B} T}=\frac{\mathrm{d} S(\rho)}{L^{d}(\rho)} k_{\left(a_{>}, b\right)}\left(\operatorname{sign}(t) L(\rho) / \xi_{ \pm}\right) & \\
& +\frac{\mathrm{d} S_{<}(\rho)}{L^{d}(\rho)} \Delta k\left(\operatorname{sign}(t) L(\rho) / \xi_{ \pm}\right)
\end{aligned}
$$

where $\Delta k(\Theta)=k_{\left(a_{<}, b\right)}(\Theta)-k_{\left(a_{>}, b\right)}(\Theta)$. Summing up all force contributions from the rings of different radii $\rho$, one finds for

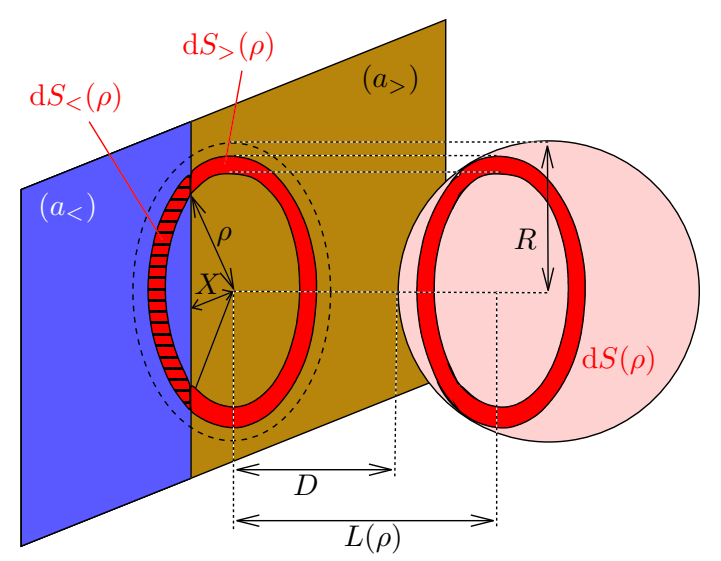

FIG. 14. Sketch concerning the Derjaguin approximation for the critical Casimir force acting on a colloid opposite to a chemical step. The critical Casimir force is subdivided into contributions from rings parallel to the substrate. The projection of the area $\mathrm{d} S(\rho)$ of a ring onto the substrate is separated into the areal contributions $\mathrm{d} S_{<}$and $\mathrm{d} S_{>}$which emerge as the intersection of the projected ring with the half-planes carrying $\left(a_{<}\right)$and $\left(a_{>}\right) \mathrm{BC}$, respectively [see the main text]. The sphere has a surface-to-surface distance $D$ from the substrate and its center has a lateral distance $X$ from the chemical step.

the total normal force acting on the sphere

$$
F_{\mathrm{S}}(X, D, R, T)=F_{\left(a_{>}, b\right)}(D, R, T)+\Delta F(X, D, R, T)
$$

where $F_{\left(a_{>}, b\right)}$ is the force acting on a sphere close to a homogeneous substrate with $\left(a_{>}\right) \mathrm{BC}$ and is given by Eq. (9) or by Eqs. (5) and (10). This term does not contribute to the lateral critical Casimir force experienced by the colloid near the chemical step, because it does not depend on the lateral coordinate of the colloid. The second term $\Delta F$ in Eq. (A3) corresponds to the integration of the force differences $\Delta k$ in the region of overlap between the projection of the sphere onto the substrate plane and that part of the substrate with $\left(a_{<}\right)$ BC. For each ring this area is given by [see Fig. 14]

$$
\mathrm{d} S_{<}(\rho)= \begin{cases}0, & \rho<X \\ 2 \arccos (X / \rho) \rho \mathrm{d} \rho, & X \leq \rho \leq R\end{cases}
$$

This leads to

$$
\frac{\Delta F(X, D, R, T)}{k_{B} T}=2 \int_{X}^{R} \mathrm{~d} \rho \rho \arccos \left(\frac{X}{\rho}\right) \frac{\Delta k\left(\operatorname{sign}(t) L(\rho) / \xi_{ \pm}\right)}{L^{d}(\rho)} .
$$

In the spirit of the DA, the radius of the sphere is taken to be large compared to its distance to the substrate, i.e., $\Delta=$ $D / R \ll 1$, and the contributions from the rings closest to the substrate dominate. Therefore, it is well justified and in accordance with the DA to assume $X / R \ll 1$ because the contributions of rings with large radii do not change the behavior of the force in the Derjaguin limit. Within these two limits we can use the parabolic approximation for the distance of the rings to the substrate [Eq. (7)], $L(\rho) \simeq D \alpha$, with $\alpha=1+\rho^{2} / 2 R D$. Changing the integration variable in Eq. A5 we directly find

$$
\Delta F(X, D, R, T)=k_{B} T \frac{R}{D^{d-1}} \Delta K(\Xi, \Theta, \Delta)
$$


where $\Delta K$ is a universal scaling function given by

$$
\Delta K(\Xi, \Theta, \Delta \rightarrow 0)=2 \int_{1+\Xi^{2} / 2}^{\infty} \mathrm{d} \alpha \alpha^{-d} \arccos \left(\frac{\Xi}{\sqrt{2(\alpha-1)}}\right) \Delta k(\alpha \Theta) .
$$

Note that the relevant scaling variable $\Xi=X / \sqrt{R D}$ can take on arbitrary values, irrespective of the two assumptions $D / R \ll 1$ and $X / R \ll 1$. From Eq. A7 one finds with Eqs. (10) and (13) directly the expression for the scaling function $\psi_{\left(a_{<} \mid a_{>}, b\right)}$ given in Eq. (16).

The critical Casimir potential $\Phi_{\mathrm{s}}(X, D, R, T)=$

$\int_{D}^{\infty} \mathrm{d} l F_{\mathrm{s}}(X, l, R, T)$ can be separated analogously to Eq. (A3), i.e.,

$$
\Phi_{\mathrm{s}}(X, D, R, T)=\Phi_{\left(a_{>}, b\right)}(D, R, T)+\Delta \Phi(X, R, D, T)
$$

with

$$
\Delta \Phi(X, R, D, T)=\int_{D}^{\infty} \mathrm{d} l \Delta F(X, l, R, T)=: k_{B} T \frac{R}{D^{d-2}} \Delta \vartheta(\Xi, \Theta, \Delta) .
$$

Using Eq. (A7), the scaling function $\Delta \vartheta$ is given by

$$
\Delta \vartheta(\Xi, \Theta, \Delta)=2 \int_{1}^{\infty} \mathrm{d} y \frac{1}{y^{d-1}} \int_{1+\Xi^{2} /(2 y)}^{\infty} \mathrm{d} \alpha \frac{1}{\alpha^{d}} \arccos \left(\frac{\Xi}{\sqrt{2 y(\alpha-1)}}\right) \Delta k(y \alpha \Theta) .
$$

By changing the integration variable $\alpha \mapsto z:=2 y(\alpha-1) / \Xi^{2}$ followed by $y \mapsto v:=y+\Xi^{2} z / 2$ one obtains

$$
\Delta \vartheta(\Xi, \Theta, \Delta)=\Xi^{2} \int_{1}^{\infty} \mathrm{d} z \int_{1+z \Xi^{2} / 2}^{\infty} \mathrm{d} v \frac{1}{v^{d}} \arccos (1 / \sqrt{z}) \Delta k(\nu \Theta)
$$

After changing the order of integration

$$
\int_{1}^{\infty} \mathrm{d} z \int_{1+z \Xi^{2} / 2}^{\infty} \mathrm{d} v=\int_{1+\Xi^{2} / 2}^{\infty} \mathrm{d} v \int_{1}^{2(v-1) / \Xi^{2}} \mathrm{~d} z
$$

and using the primitive ${ }^{62}$

$$
\int \mathrm{d} z \arccos (1 / \sqrt{z})=z \arccos (1 / \sqrt{z})-\sqrt{z-1}+c
$$

one obtains after a final change of variables $v \mapsto w:=2(v-1) / \Xi^{2}$

$$
\Delta \vartheta(\Xi, \Theta, \Delta)=\frac{\Xi^{4}}{2} \int_{1}^{\infty} \mathrm{d} s \frac{1}{\left(1+\Xi^{2} s / 2\right)^{d}}\left[s \arccos \left(s^{-1 / 2}\right)-\sqrt{s-1}\right] \Delta k\left(\Theta\left[1+\Xi^{2} s / 2\right]\right) .
$$

From Eq. (A14) together with Eq. (11) one obtains the final expression for the scaling function of the critical Casimir potential as given in Eq. (18).

\section{Bulk critical point: $\Theta=0$}

In order to calculate the critical Casimir force acting on the colloid at the bulk critical point one inserts Eq. (2) into Eq. (A7) and obtains

$$
\begin{aligned}
\Delta K(\Xi, \Theta=0, \Delta) & =2\left(\Delta_{\left(a_{<}, b\right)}-\Delta_{\left(a_{>}, b\right)}\right) \int_{1+\Xi^{2} / 2}^{\infty} \mathrm{d} \alpha \alpha^{-d} \arccos \left(\frac{\Xi}{\sqrt{2 \alpha-2}}\right) \\
& =: \Xi^{2}\left(\Delta_{\left(a_{<}, b\right)}-\Delta_{\left(a_{>}, b\right)}\right) I_{d}\left(\Xi^{2} / 2\right),
\end{aligned}
$$

where $\Delta_{(a, b)}=k_{(a, b)}(0)$ [see Eq. (2)], and with the substitution $\alpha \mapsto z=\Xi / \sqrt{2(\alpha-1)}$ for $d>1$,
For $I_{d}(a)$ the recursion relation

$$
I_{d+1}(a)=\frac{1}{d} a^{1-d} \frac{\mathrm{d}}{\mathrm{d} a}\left[a^{d} I_{d}(a)\right]
$$

$$
I_{d}(a)=2 \int_{0}^{1} \mathrm{~d} z \frac{z^{2 d-3}}{\left(z^{2}+a\right)^{d}} \arccos (z)
$$

holds, so that $I_{4}$ and $I_{3}$ can be expressed in terms of $I_{2}$. Per- 
forming the integration we find ${ }^{63}$

$$
I_{2}(a)=\frac{\pi}{2 a}\left[1-\frac{a^{1 / 2}}{(1+a)^{1 / 2}}\right]
$$

and therefore with Eq. A17

$$
I_{3}(a)=\frac{\pi}{4 a}\left[1-\frac{\frac{3}{2} a^{1 / 2}+a^{3 / 2}}{(1+a)^{3 / 2}}\right]
$$

and

$$
I_{4}(a)=\frac{\pi}{6 a}\left[1-\frac{\frac{15}{8} a^{1 / 2}+\frac{5}{2} a^{3 / 2}+a^{5 / 2}}{(1+a)^{5 / 2}}\right] .
$$

Thus, from Eqs. (A16), A19), and (A20) together with the expression for $K_{\left(a_{\gtrless}, b\right)}(0,0)=2 \pi \Delta_{\left(a_{\gtrless}, b\right)} /(d-1)$ [Sec. [IIA] and Eq. 13), one finds the expression for the scaling function $\psi_{\left(a_{<} \mid a_{>}, b\right)}$ given in Eq. (17). The critical Casimir potential at
$\Theta=0$ for $d=3$ and 4 can be found from Eq. A10 together with Eq. A16:

$$
\Delta \vartheta(\Xi, \Theta=0, \Delta)=\Xi^{2}\left(\Delta_{\left(a_{<}, b\right)}-\Delta_{\left(a_{>}, b\right)}\right) \int_{1}^{\infty} \mathrm{d} y y^{-d} I_{d}\left(\frac{\Xi^{2}}{2 y}\right),
$$

and from a change of variable $y \mapsto a=\Xi^{2} /(2 y)$ one finds

$$
\Delta \vartheta(\Xi, 0, \Delta)=\frac{2^{d-2}}{\Xi^{2 d-4}}\left(\Delta_{\left(a_{<}, b\right)}-\Delta_{\left(a_{>}, b\right)}\right) \int_{0}^{\Xi^{2} / 2} \mathrm{~d} a a^{d-2} I_{d}(a) .
$$

Using Eq. A17) and the limiting behavior $I_{d}(a \rightarrow 0)=$ $\pi /(2(d-1) a)$, we find

$$
\Delta \vartheta(\Xi, 0, \Delta)=\frac{\Xi^{2}}{2(d-1)}\left(\Delta_{\left(a_{<}, b\right)}-\Delta_{\left(a_{>}, b\right)}\right) I_{d-1}\left(\Xi^{2} / 2\right) .
$$

From Eqs. (A18), (A19), and (A23) together with $\vartheta(0,0)$ as given in Sec. III A one obtains Eq. (19) for the scaling function of the critical Casimir potential at $T_{c}$.

\section{Far from criticality: $\Theta \gg 1$}

Far from the critical point, i.e., for $\Theta \gg 1$, and for symmetry breaking boundary conditions $\left(a_{<}\right)=(+),\left(a_{>}\right)=(-)$, and $(b)=(-)$ Eq. (3) holds and the integrals in Eqs. (A7) and (A14) can be calculated analytically. For $\Theta \gg 1$ Eq. (A7) turns into

$$
\Delta K(\Xi, \Theta \gg 1, \Delta)=2\left(A_{-}-A_{+}\right) \Theta^{d} \int_{1+\Xi^{2} / 2}^{\infty} \mathrm{d} \alpha \arccos \left(\frac{\Xi}{\sqrt{2(\alpha-1)}}\right) e^{-\alpha \Theta} .
$$

Substituting $\alpha \mapsto \beta=2(\alpha-2) / \Xi^{2}$ one has

$$
\Delta K(\Xi, \Theta \gg 1, \Delta)=\Xi^{2}\left(A_{-}-A_{+}\right) \Theta^{d} e^{-\Theta} \int_{1}^{\infty} \mathrm{d} \beta \arccos \left(\beta^{-1}\right) e^{-\Xi^{2} \Theta \beta / 2} .
$$

Integrating by parts leads to

$$
\Delta K(\Xi, \Theta \gg 1, \Delta)=\left(A_{-}-A_{+}\right) \Theta^{d-1} e^{-\Theta} \int_{1}^{\infty} \mathrm{d} \beta \frac{1}{\beta \sqrt{\beta-1}} e^{-\Xi^{2} \Theta \beta / 2} .
$$

By using the relation ${ }^{64}$

$$
\int_{1}^{\infty} \mathrm{d} \beta \frac{1}{\beta \sqrt{\beta-1}} e^{-a^{2} \beta}=\pi \operatorname{erfc}(a),
$$

where $a>0$ and $\operatorname{erfc}(a)=1-\operatorname{erf}(a)=2 \pi^{-1 / 2} \int_{a}^{\infty} \mathrm{d} t \exp \left(-t^{2}\right)$ is the complementary error function, we finally arrive at

$$
\Delta K(\Xi, \Theta \gg 1, \Delta)=\pi\left(A_{-}-A_{+}\right) \Theta^{d-1} e^{-\Theta} \operatorname{erfc}(\Xi \sqrt{\Theta / 2}) .
$$

The scaling function $K_{(\mp,-)}$ for $\Theta \gg 1$ in the homogeneous case [Sec. [II]] is given by $\underline{\underline{7}}$

$$
K_{(\mp,-)}(\Theta \gg 1, \Delta \rightarrow 0)=2 \pi A_{ \pm} \Theta^{d-1} e^{-\Theta}
$$

and from Eqs. (13), (A28), and (A29) one obtains the expression for $\psi_{(-\mid+,-)}$as given in Eq. (20). Similarly, after rewriting Eq. (A14) for $\Theta \gg 1$ as

$$
\Delta \vartheta(\Xi, \Theta \gg 1, \Delta)=\left(A_{-}-A_{+}\right) \Theta^{d} e^{-\Theta} \frac{\Xi^{4}}{2} \int_{1}^{\infty} \mathrm{d} s\left(s \arccos \left(s^{-1 / 2}\right)-\sqrt{s-1}\right) e^{-\Xi^{2} \Theta s / 2},
$$

one can integrate by parts, which yields

$$
\Delta \vartheta(\Xi, \Theta \gg 1, \Delta)=\left(A_{-}-A_{+}\right) \Theta^{d-2} e^{-\Theta} \int_{1}^{\infty} \mathrm{d} s \frac{1}{\sqrt{s-1}}\left[\frac{1}{s}+\frac{\Theta \Xi^{2}}{2}\left(1+\Theta \Xi^{2}\right)-\frac{\Theta^{2} \Xi^{4}}{2} s\right] e^{-\Xi^{2} \Theta s / 2} .
$$


Using Eq. A27) and the relations [which follow from taking successive derivatives $-d / d\left(a^{2}\right)$ of Eq. A27]

$$
\int_{1}^{\infty} \mathrm{d} s \frac{1}{\sqrt{s-1}} e^{-a^{2} s}=\frac{\sqrt{\pi}}{a} e^{-a^{2}}, \quad \int_{1}^{\infty} \mathrm{d} s \frac{s}{\sqrt{s-1}} e^{-a^{2} s}=\frac{\sqrt{\pi}}{2 a^{3}}\left(1+2 a^{2}\right) e^{-a^{2}}
$$

one ends up with

$$
\Delta \vartheta(\Xi, \Theta \gg 1, \Delta)=\pi\left(A_{-}-A_{+}\right) \Theta^{d-2} e^{-\Theta} \operatorname{erfc}(\Xi \sqrt{\Theta / 2}) .
$$

Together with the expression for the homogeneous case [see Sec. III] and Ref.7],

$$
\vartheta_{(\mp,-)}(\Theta \gg 1, \Delta \rightarrow 0)=2 \pi A_{ \pm} \Theta^{d-2} e^{-\Theta},
$$

one obtains the expression for $\omega_{(-\mid+,-)}$given in Eq. (20).

\section{Appendix B: Derjaguin approximation for a single chemical lane}

Based on the assumption of additivity which underlies the Derjaguin approximation one can use the results presented in Sec.IV for a chemical step in order to study a chemical lane. The chemical lane configuration can be regarded as the superposition of two chemical steps, one $(A)$ being a chemical step located at $x=-L$ with $\left(a \mid a_{\ell}\right) \mathrm{BC}$, and the other one $(B)$ being a chemical step located at $x=L$ with $\left(a_{\ell} \mid a\right)$ BC. This superposition overcounts a contribution corresponding to a homogeneous substrate with $\left(a_{\ell}\right)$ BC which must be subtracted [see Eq. [13)]:

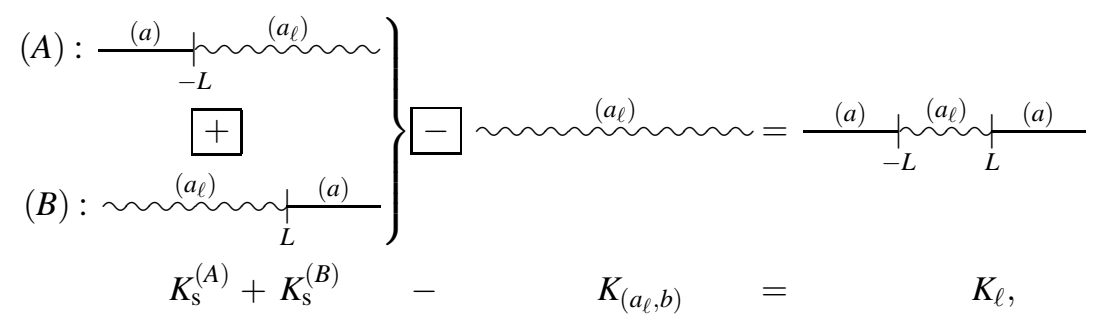

where

$$
K_{\mathrm{s}}^{(A)}(\Lambda, \Xi, \Theta, \Delta)=\frac{K_{(a, b)}+K_{\left(a_{\ell}, b\right)}}{2}+\frac{K_{(a, b)}-K_{\left(a_{\ell}, b\right)}}{2} \psi_{\left(a \mid a_{\ell}, b\right)}(\Xi+\Lambda, \Theta, \Delta)
$$

and

$$
K_{\mathrm{S}}^{(B)}(\Lambda, \Xi, \Theta, \Delta)=\frac{K_{(a, b)}+K_{\left(a_{\ell}, b\right)}}{2}+\frac{K_{\left(a_{\ell}, b\right)}-K_{(a, b)}}{2} \psi_{\left(a_{\ell} \mid a, b\right)}(\Xi-\Lambda, \Theta, \Delta) .
$$

Since within the DA $\psi_{\left(a_{\ell} \mid a, b\right)}=\psi_{\left(a \mid a_{\ell}, b\right)}$, Eqs. (B1)-(B) and Eq. (27) lead directly to Eq. (29). The procedure for calculating the critical Casimir potential is analogous to the one discussed here for the force and leads to Eq. (30).

\section{Appendix C: Derjaguin approximation for periodic chemical patterns}

In order to obtain the scaling function for the critical Casimir force and the potential of a sphere close to a periodic chemical pattern one can follow a procedure analogous to the one presented in Appendix B Indeed, in order to form a lane $\ell^{\prime}$ with $\left(a_{1}\right)$ $\mathrm{BC}$ on an otherwise homogeneous portion of a substrate with $\left(a_{2}\right) \mathrm{BC}$ and lateral extension $P$, one can proceed as follows:

(A): superimpose onto the substrate the single chemical lane $\ell$ studied in Sec. $\nabla$, with $a_{\ell}=a_{1}, a=a_{2}$, suitably positioned in space such that it coincides with the lane $\ell^{\prime}$ to be formed.

(B): subtract the contribution of a homogeneous substrate with $\left(a_{2}\right) \mathrm{BC}$, which is overcounted in the previous superposition. After this subtraction, the contribution to the force resulting from that part - marked by (?) in Eq. ( $\overline{C 1}$ - of the original substrate which is not affected by the formation of the extra lane is unchanged. 


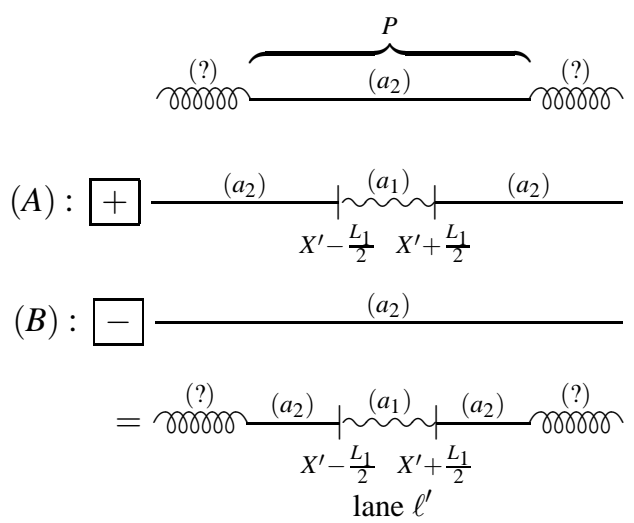

The contribution $\Delta F$ to the critical Casimir force experienced by a colloid close to such a substrate and due to the addition of the lane is characterized by the scaling function [see Eq. 27)]

$$
\begin{aligned}
\Delta K\left(\lambda, \Pi, \Xi-\Xi^{\prime}, \Theta, \Delta \rightarrow 0\right)=K_{\ell}\left(\Pi \frac{\lambda}{2}, \Xi-\Xi^{\prime}, \Theta\right)-K_{\left(a_{2}, b\right)} & =\frac{K_{\left(a_{2}, b\right)}-K_{\left(a_{1}, b\right)}}{2} \\
& \times\left[\psi_{\left(a_{1} \mid a, b\right)}\left(\Xi-\Xi^{\prime}+\Pi \frac{\lambda}{2}, \Theta, \Delta \rightarrow 0\right)-\psi_{\left(a_{1} \mid a, b\right)}\left(\Xi-\Xi^{\prime}-\Pi \frac{\lambda}{2}, \Theta, \Delta \rightarrow 0\right)\right]
\end{aligned}
$$

where we have used the relation $\left(L_{1} / 2\right) / \sqrt{R D}=\Pi \lambda / 2$ and have introduced $\Xi^{\prime} \equiv X^{\prime} / \sqrt{R D}$, with $X^{\prime}$ as the position of the center of the added lane $\ell^{\prime}$. The force resulting from a periodic pattern can now be obtained by starting out with a homogeneous substrate with $\left(a_{2}\right) \mathrm{BC}$ and by iterating the procedure discussed above which adds progressively displaced lanes at positions $X^{\prime}=n P$, i.e., $\Xi^{\prime}=n \Pi$, with $n \in \mathbb{Z}$. The resulting force is characterized by the scaling function

$$
K_{\mathrm{p}}(\lambda, \Pi, \Xi, \Theta, \Delta \rightarrow 0)=K_{\left(a_{2}, b\right)}+\sum_{n=-\infty}^{+\infty} \Delta K(\lambda, \Pi, \Xi-n \Pi, \Theta, \Delta \rightarrow 0)
$$

which, together with Eq. (33), yields immediately Eq. (35) for $\psi_{\mathrm{p}}$.

For $\lambda=0$ or $\lambda=1$ one recovers from Eq. (35) the homogeneous cases with $\left(a_{2}, b\right)$ BC or $\left(a_{1}, b\right)$ BC, respectively. Obviously, for $\lambda=0$, the sum in Eq. (35) vanishes, and one is left with $\psi_{\mathrm{p}}(\lambda=0, \Pi, \Xi, \Theta, \Delta \rightarrow 0)=1$, corresponding to $\left(a_{2}, b\right) \mathrm{BC}$. On the other hand for $\lambda=1$, the sum in Eq. (35) can be easily evaluated [see Eq. (16) for $|\Xi| \rightarrow \infty$ ]:

$$
\begin{aligned}
\lim _{M, N \rightarrow \infty} \sum_{n=-M}^{N}\left\{\psi_{\left(a_{1} \mid a_{2}, b\right)}(\Xi+\Pi(n\right. & \left.\left.\left.+\frac{1}{2}\right), \Theta, \Delta\right)-\psi_{\left(a_{1} \mid a_{2}, b\right)}\left(\Xi+\Pi\left(n-\frac{1}{2}\right), \Theta, \Delta\right)\right\} \\
& =\lim _{M, N \rightarrow \infty}\left\{\psi_{\left(a_{1} \mid a_{2}, b\right)}\left(\Xi+\Pi\left(N+\frac{1}{2}\right), \Theta, \Delta\right)-\psi_{\left(a_{1} \mid a_{2}, b\right)}\left(\Xi+\Pi\left(-M-\frac{1}{2}\right), \Theta, \Delta\right)\right\}=-2
\end{aligned}
$$

where we have used the fact that $\psi_{\left(a_{1} \mid a_{2}, b\right)}(\Xi= \pm \infty, \Theta, \Delta)=\mp 1$. Accordingly, $\psi_{\mathrm{p}}(\lambda=1, \Pi, \Xi, \Theta, \Delta \rightarrow 0)=-1$, which corresponds to the homogeneous case with $\left(a_{1}, b\right) \mathrm{BC}$.

In the limit $\Pi \rightarrow 0$ (i.e., for very fine patterns compared with $\sqrt{R D}$ ), the sum in Eq. (35) turns into an integral:

$$
\begin{gathered}
\sum_{n=-\infty}^{\infty}\left\{\psi_{\left(a_{1} \mid a_{2}, b\right)}\left(\Xi+\Pi\left(n+\frac{\lambda}{2}\right), \Theta, \Delta\right)-\psi_{\left(a_{1} \mid a_{2}, b\right)}\left(\Xi+\Pi\left(n-\frac{\lambda}{2}\right), \Theta, \Delta\right)\right\} \\
\underset{\Pi \rightarrow 0}{\longrightarrow} \frac{1}{\Pi} \int_{-\infty}^{\infty} \mathrm{d} \eta\left\{\psi_{\left(a_{1} \mid a_{2}, b\right)}\left(\Xi+\eta+\frac{\Pi \lambda}{2}, \Theta, \Delta\right)-\psi_{\left(a_{1} \mid a_{2}, b\right)}\left(\Xi+\eta-\frac{\Pi \lambda}{2}, \Theta, \Delta\right)\right\} \\
=\int_{-\infty}^{\infty} \mathrm{d} \eta \lambda \frac{\mathrm{d}}{\mathrm{d} \eta} \psi_{\left(a_{1} \mid a_{2}, b\right)}(\Xi+\eta, \Theta, \Delta) \\
=\lambda\left\{\psi_{\left(a_{1} \mid a_{2}, b\right)}(+\infty, \Theta, \Delta)-\psi_{\left(a_{1} \mid a_{2}, b\right)}(-\infty, \Theta, \Delta)\right\}=-2 \lambda
\end{gathered}
$$

and finally one finds Eq. (36).

For completeness, we provide the corresponding expression for the scaling function of the critical Casimir potential $\omega_{\mathrm{p}}$ within the DA:

$$
\omega_{\mathrm{p}}(\lambda, \Pi, \Xi, \Theta, \Delta \rightarrow 0)=1+\sum_{n=-\infty}^{\infty}\left\{\omega_{\left(a_{1} \mid a_{2}, b\right)}\left(\Xi+\Pi\left(n+\frac{\lambda}{2}\right), \Theta, \Delta \rightarrow 0\right)-\omega_{\left(a_{1} \mid a_{2}, b\right)}\left(\Xi+\Pi\left(n-\frac{\lambda}{2}\right), \Theta, \Delta \rightarrow 0\right)\right\} .
$$

In the limit $\Pi \rightarrow 0, \omega_{\mathrm{p}}$ reduces to

$$
\omega_{\mathrm{p}}(\lambda, \Pi \rightarrow 0, \Xi, \Theta, \Delta \rightarrow 0)=1-2 \lambda
$$


Accordingly, within the DA and in the limit $\Pi \rightarrow 0$ the critical Casimir potential is the average of the ones corresponding to the two boundary conditions, weighted with the corresponding relative stripe width:

$$
\vartheta_{\mathrm{p}}(\lambda, \Pi \rightarrow 0, \Xi, \Theta, \Delta \rightarrow 0)=\lambda \vartheta_{\left(a_{1}, b\right)}(\Theta, \Delta \rightarrow 0)+(1-\lambda) \vartheta_{\left(a_{2}, b\right)}(\Theta, \Delta \rightarrow 0) .
$$

\section{Appendix D: Cylinder close to a patterned substrate}

\section{Derjaguin approximation for a homogeneous substrate}

Similarly to the case of a sphere discussed before, the critical Casimir force $F_{(a, b)}^{\mathrm{cyl}}$ per unit length acting on a (threedimensional) cylinder of radius $R$ with $(b) \mathrm{BC}$ close to and parallel to a substrate with $(a) \mathrm{BC}$ at a surface-to-surface distance $D$ can be expressed in terms of a universal scaling function $K^{\text {cyl. }}$

$$
F_{(a, b)}^{\mathrm{cyl}}(D, R, T)=k_{B} T \frac{R^{1 / 2}}{D^{d-1 / 2}} K_{(a, b)}^{\mathrm{cyl}}(\Theta, \Delta),
$$

with $\Theta=\operatorname{sign}(t) D / \xi_{ \pm}$and $\Delta=D / R$ as before. Equation (D1) describes a force divided by a length and per $D^{d-3}$ which for $d=4$ corresponds to considering $F_{(a, b)}^{\mathrm{cyl}}$ per length of its axis and per length of the extra translationally invariant direction of a hypercylinder [compare Eq. (5)]. The geometric prefactor in Eq. (D1), however, differs from the one for the sphere [Eq. (5)] because it is chosen such that within the DA $(\Delta \rightarrow 0)$ the scaling function $K_{(a, b)}^{\mathrm{cyl}}$ attains a nonzero and finite limit, as discussed before. The DA can be implemented along the lines of Sec. IIIA for the sphere. Here the surface of the cylindrical colloid is decomposed into pairs of infinitely narrow stripes of combined area $\mathrm{d} S=2 M \mathrm{~d} \rho$, positioned parallel to the substrate at a distance $L(\rho)$ from it [Eq. (7)] and each at a distance $\rho$ from the symmetry plane of the configuration. $M$ is the length of the cylinder and drops out from $F_{(a, b)}^{\mathrm{cyl}}$ which follows analogously from Eqs. (8) and (9):

$F_{(a, b)}^{\mathrm{cyl}}(D, R, T) / k_{B} T \simeq 2 \int_{0}^{R} \mathrm{~d} \rho[L(\rho)]^{-d} k_{(a, b)}\left(\operatorname{sign}(t) L(\rho) / \xi_{ \pm}\right)$,

where $L(\rho)$ is given in Eq. (7). Finally, in the limit $\Delta \rightarrow 0$ we obtain

$$
K_{(a, b)}^{\mathrm{cyl}}(\Theta, \Delta \rightarrow 0)=\sqrt{2} \int_{1}^{\infty} \mathrm{d} \alpha(\alpha-1)^{-\frac{1}{2}} \alpha^{-d} k_{(a, b)}(\Theta \alpha) .
$$

At the bulk critical point $\Theta=0$ one finds $K_{(a, b)}^{\mathrm{cyl}}(0,0)=\sqrt{2 \pi}\left[\Gamma\left(d-\frac{1}{2}\right) / \Gamma(d)\right] \Delta_{(a, b)} \quad$ so $\quad$ that $K_{(a, b)}^{\mathrm{cyl}}(0,0)=[3 \pi /(4 \sqrt{2})] \Delta_{(a, b)} \simeq 1.66 \times \Delta_{(a, b)}$ for $d=3$ and $K_{(a, b)}^{\mathrm{cyl}}(0,0)=[5 \pi /(8 \sqrt{2})] \Delta_{(a, b)} \simeq 1.38 \times \Delta_{(a, b)}$ for $d=4$.

\section{Derjaguin approximation for a chemical step}

Here, we assume that the axis of the cylinder is parallel to the chemical step, i.e., perpendicular to the $x$ direction [Fig.1], as well as parallel to the substrate. The projection of the position of the axis of the cylinder with respect to the $x$ direction is denoted by $X$, so that at $X=0$ the cylinder is positioned directly above the chemical step [Fig. 1]. Accordingly, the problem is effectively two-dimensional and the corresponding DA can be performed much easier than in Appendix A Following an approach analogous to the one adopted for the sphere in Sec.IV and in Appendix $\mathrm{A}$ we rewrite the normal critical Casimir force per unit length acting on the cylinder as in Eq. (A3):

$$
F_{\mathrm{s}}^{\mathrm{cyl}}(X, D, R, T)=F_{\left(a_{>}, b\right)}^{\mathrm{cyl}}(D, R, T)+\Delta F^{\mathrm{cyl}}(X, D, R, T) .
$$

Within the DA we find for $\Delta \rightarrow 0$ [compare Eq. (A6)]

$$
\Delta F^{\mathrm{cyl}}(X, D, R, T)=k_{B} T \frac{R^{1 / 2}}{D^{d-1 / 2}} \Delta K^{\mathrm{cyl}}(\Xi, \Theta, \Delta \rightarrow 0),
$$

where [compare Eq. A7] ]

$\Delta K^{\mathrm{cyl}}(\Xi, \Theta, \Delta \rightarrow 0)=\sqrt{2} \int_{1+\Xi^{2} / 2}^{\infty} \mathrm{d} \alpha(\alpha-1)^{-\frac{1}{2}} \alpha^{-d} \Delta k(\Theta \alpha)$.

Using Eq. (D6) and Eq. (D3) we find for the whole range of values of $\Xi$ the scaling function $\psi_{\left(a_{<} \mid a_{>}, b\right)}^{\text {cyl }}$ which is defined completely analogous to Eq. (13) [compare Eq. (16)]:

$$
\begin{aligned}
\psi_{\left(a_{<} \mid a_{>}, b\right)}^{\mathrm{cyl}}(\Xi \gtrless 0, \Theta, \Delta \rightarrow 0)=\mp 1 \\
\quad \pm \frac{\sqrt{2} \int_{1+\Xi^{2} / 2}^{\infty} \mathrm{d} \alpha(\alpha-1)^{-\frac{1}{2}} \alpha^{-d} \Delta k(\Theta \alpha)}{K_{\left(a_{<}, b\right)}^{\mathrm{cyl}}(\Theta, \Delta \rightarrow 0)-K_{\left(a_{>}, b\right)}^{\mathrm{cyl}}(\Theta, \Delta \rightarrow 0)} .
\end{aligned}
$$

\section{Derjaguin approximation for a periodic chemical pattern}

The derivation of the scaling function for the critical Casimir force acting on the cylinder close to and aligned with a periodic chemical pattern as studied in Sec.VII is analogous to the one for the sphere described in Appendix $\mathrm{C}$ The final formula for $\psi_{\mathrm{p}}^{\mathrm{cyl}}$ is the same as in Eq. (35) with $\psi_{\left(a_{1} \mid a_{2}, b\right)}$ replaced by $\psi_{\left(a_{1} \mid a_{2}, b\right)}^{\mathrm{cyl}}$ given by Eq. (D7). This renders the critical Casimir force per unit length

$$
F_{\mathrm{p}}^{\mathrm{cyl}}\left(L_{1}, P, X, D, R, T\right)=k_{B} T \frac{R^{1 / 2}}{D^{d-1 / 2}} K_{\mathrm{p}}^{\mathrm{cyl}}(\lambda, \Pi, \Xi, \Theta, \Delta)
$$

where $K_{\mathrm{p}}^{\mathrm{cyl}}$ is defined as in Eq. (33) with $K_{\left(a_{1}, b\right)}$ and $K_{\left(a_{2}, b\right)}$ replaced by $K_{\left(a_{1}, b\right)}^{\mathrm{cyl}}$ and $K_{\left(a_{2}, b\right)}^{\mathrm{cyl}}$, respectively, which are given by Eq. (D3), and with $\psi_{\mathrm{p}}$ replaced by $\psi_{\mathrm{p}}^{\mathrm{cyl}}$. The corresponding results are shown in Fig. 12 
${ }^{1}$ H. G. B. Casimir, Proc. K. Ned. Akad. Wet. 51, 793 (1948).

${ }^{2}$ M. Kardar and R. Golestanian, Rev. Mod. Phys. 71, 1233 (1999).

${ }^{3}$ M. E. Fisher and P. G. de Gennes, C. R. Acad. Sci., Paris, Ser. B 287, 207 (1978).

${ }^{4}$ A. Gambassi, J. Phys.: Conf. Ser. 161, 012037 (2009).

${ }^{5}$ A. Gambassi, C. Hertlein, L. Helden, C. Bechinger, and S. Dietrich, Europhysics News 40/1, 18 (2009).

${ }^{6}$ C. Hertlein, L. Helden, A. Gambassi, S. Dietrich, and C. Bechinger, Nature 451, 172 (2008).

${ }^{7}$ A. Gambassi, A. Maciołek, C. Hertlein, U. Nellen, L. Helden, C. Bechinger, and S. Dietrich, Phys. Rev. E 80, 061143 (2009).

${ }^{8} \mathrm{M}$. Krech, The Casimir Effect in Critical Systems (World Scientific, Singapore, 1994).

${ }^{9}$ J. G. Brankov, D. M. Danchev, and N. S. Tonchev, Theory of critical phenomena in finite size systems (World Scientific, Singapore, 2000).

${ }^{10}$ M. Krech and S. Dietrich, Phys. Rev. Lett. 66, 345 (1991); Phys. Rev. A 46, 1886 (1992); ibid 1922 (1992).

${ }^{11}$ R. Evans and J. Stecki, Phys. Rev. B 49, 8842 (1994).

${ }^{12}$ H. W. Diehl, D. Grüneberg, and M. A. Shpot, EPL 75, 241 (2006).

${ }^{13}$ R. Zandi, A. Shackell, J. Rudnick, M. Kardar, and L. P. Chayes, Phys. Rev. E 76, 030601 (2007).

${ }^{14}$ F. M. Schmidt and H. W. Diehl, Phys. Rev. Lett. 101, 100601 (2008).

${ }^{15}$ T. F. Mohry, A. Maciołek, and S. Dietrich, arXiv:1004.0112 (2010); T. F. Mohry, diploma thesis, University of Stuttgart (2008).

${ }^{16}$ J. N. Munday, F. Capasso, and V. A. Parsegian, Nature 457, 170 (2009).

${ }^{17}$ R. Garcia and M. H. W. Chan, Phys. Rev. Lett. 83, 1187 (1999); ibid 88, 086101 (2002).

${ }^{18}$ A. Ganshin, S. Scheidemantel, R. Garcia, and M. H. W. Chan, Phys. Rev. Lett. 97, 075301 (2006).

${ }^{19}$ M. Fukuto, Y. F. Yano, and P. S. Pershan, Phys. Rev. Lett. 94, 135702 (2005).

${ }^{20}$ S. Rafai, D. Bonn, and J. Meunier, Physica A 386, 31 (2007).

${ }^{21}$ A. Hucht, Phys. Rev. Lett. 99, 185301 (2007).

${ }^{22}$ O. Vasilyev, A. Gambassi, A. Maciołek, and S. Dietrich, EPL 80, 60009 (2007).

${ }^{23}$ O. Vasilyev, A. Gambassi, A. Maciołek, and S. Dietrich, Phys. Rev. E 79, 041142 (2009); ibid 80, 039902(E) (2009).

${ }^{24}$ M. Hasenbusch, J. Stat. Mech., P07031 (2009); Phys. Rev. E 80, 061120 (2009); arXiv:0907.2847 (2009).

${ }^{25}$ T. W. Burkhardt and E. Eisenriegler, Phys. Rev. Lett. 74, 3189 (1995); ibid 78, 2867 (1997).

${ }^{26}$ E. Eisenriegler and U. Ritschel, Phys. Rev. B 51, 13717 (1995).

${ }^{27}$ A. Hanke, F. Schlesener, E. Eisenriegler, and S. Dietrich, Phys. Rev. Lett. 81, 1885 (1998).

${ }^{28}$ F. Schlesener, A. Hanke, and S. Dietrich, J. Stat. Phys. 110, 981 (2003).

${ }^{29}$ E. Eisenriegler, J. Chem. Phys. 121, 3299 (2004).

${ }^{30}$ S. Kondrat, L. Harnau, and S. Dietrich, J. Chem. Phys. 131, 204902 (2009).

${ }^{31}$ M. Tröndle, S. Kondrat, A. Gambassi, L. Harnau, and S. Dietrich, EPL 88, 40004 (2009).

${ }^{32}$ F. Soyka, O. Zvyagolskaya, C. Hertlein, L. Helden, and C. Bechinger, Phys. Rev. Lett. 101, 208301 (2008)

${ }^{33}$ M. Sprenger, F. Schlesener, and S. Dietrich, J. Chem. Phys. 124, 134703 (2006).

${ }^{34}$ M. Tröndle, L. Harnau, and S. Dietrich, J. Chem. Phys. 129, 124716 (2008).

${ }^{35}$ M. Krech, Phys. Rev. E 56, 1642 (1997).

${ }^{36}$ U. Leonhardt and T. G. Philbin, New J. Phys. 9, 254 (2007).

${ }^{37}$ A. W. Rodriguez, J. N. Munday, J. D. Joannopoulos, F. Capasso, D. A. R. Dalvit, and S. G. Johnson, Phys. Rev. Lett. 101, 190404 (2008).

${ }^{38}$ A. W. Rodriguez, A. P. McCauley, D. Woolf, F. Capasso, J. D. Joannopoulos, and S. G. Johnson, Phys. Rev. Lett. 104, 160402 (2010).
${ }^{39}$ S. J. Rahi, M. Kardar, and T. Emig, arXiv:0911.5364 (2009).

${ }^{40}$ S. J. Rahi and S. Zaheer, arXiv:0909.4510 (2009).

${ }^{41}$ R. Zhao, J. Zhou, T. Koschny, E. N. Economou, and C. M. Soukoulis, Phys. Rev. Lett. 103, 103602 (2009).

${ }^{42} \mathrm{~K}$. Binder, in Phase Transitions and Critical Phenomena, edited by C. Domb and J. L. Lebowitz (Academic, London, 1983), Vol. 8, p. 1.

${ }^{43} \mathrm{H}$. W. Diehl, in Phase Transitions and Critical Phenomena, edited by C. Domb and J. L. Lebowitz (Academic, London, 1986), Vol. 10, p. 75.

${ }^{44}$ H. W. Diehl, Int. J. Mod. Phys. B 11, 3503 (1997).

${ }^{45}$ A. Pelissetto and E. Vicari, Phys. Rep. 368, 549 (2002).

${ }^{46}$ V. Privman, P. C. Hohenberg, and A. Aharony, in Phase Transitions and Critical Phenomena, edited by C. Domb and J. L. Lebowitz (Academic, London, 1991), Vol. 14, p. 1 and p. 364.

${ }^{47}$ H. B. Tarko and M. E. Fisher, Phys. Rev. Lett. 31, 926 (1973); Phys. Rev. B 11, 1217 (1975).

${ }^{48}$ Z. Borjan and P. J. Upton, Phys. Rev. Lett. 101, 125702 (2008).

${ }^{49}$ T. W. Burkhardt and H. W. Diehl, Phys. Rev. B 50, 3894 (1994).

${ }^{50}$ H. W. Diehl and M. Smock, Phys. Rev. B 47, 5841 (1993); ibid 48, 6740 (1993).

${ }^{51}$ B. Derjaguin, Kolloid Z. 69, 155 (1934).

${ }^{52}$ For the scaling function in $d=3$ of the critical Casimir force acting on two parallel planar walls with $( \pm) \mathrm{BC}$, we use the approximation denoted by (i) in Figs. 9 and 10 of Ref. 23. The uncertainty of the overall amplitude of the scaling functions is about $10 \%$ to $20 \%$ as indicated by the different results obtained by the various approximations used in Ref. 23. Correspondingly, this uncertainty affects our predictions for the scaling functions $K_{( \pm,-)}, \vartheta_{( \pm,-)}, K_{\mathrm{s}}^{\|}, K_{\mathrm{p}}, \Phi_{\mathrm{p}}$, and $K_{\mathrm{p}}^{\text {cyl }}$ based on such Monte Carlo simulation data. However, the normalized scaling functions $\omega_{(+\mid-,-)}, \omega_{\ell}, \psi_{\mathrm{p}}$, and $\psi_{\mathrm{p}}^{\text {cyl }}$ are affected less leading to an uncertainty of at most $3 \%$.

${ }^{53}$ U. Nellen, L. Helden, and C. Bechinger, EPL 88, 26001 (2009).

${ }^{54} \mathrm{D}$. Vogt, diploma thesis, University of Stuttgart (2009).

${ }^{55}$ Private communication by D. Vogt, O. Zvyagolskaya, and C. Bechinger.

${ }^{56}$ A. W. Rodriguez, D. Woolf, A. P. McCauley, F. Capasso, J. D. Joannopoulos, and S. G. Johnson, arXiv:1004.2733 (2010).

${ }^{57}$ M. Hoffmann, Y. Lu, M. Schrinner, M. Ballauff, and L. Harnau, J. Phys. Chem. B 112, 14843 (2008); M. Hoffmann, M. Siebenbürger, L. Harnau, M. Hund, C. Hanske, Y. Lu, C. S. Wagner, M. Drechsler, and M. Ballauff, Soft Matter 6, 1125 (2010).

${ }^{58}$ M. Sprenger, F. Schlesener, and S. Dietrich, Phys. Rev. E 71, 056125 (2005).

${ }^{59}$ D. Dantchev, H. W. Diehl, and D. Gruneberg, Phys. Rev. E 73, 016131 (2006).

${ }^{60}$ D. Dantchev, F. Schlesener, and S. Dietrich, Phys. Rev. E 76, 011121 (2007).

${ }^{61}$ A. Maciołek, A. Gambassi, and S. Dietrich, Phys. Rev. E 76, 031124 (2007).

${ }^{62}$ See Eq. 7.8 .3 on p. 168 in Tables of indefinite integrals, edited by Y. A. Brychkov, O. I. Marichev, and A. P. Prudnikov (Gordon and Breach, New York, 1989), with the substitution $z \mapsto x=1 / \sqrt{z}$.

${ }^{63}$ See Eq. (4) of Tab. 234 in Nouvelles tables d'intégrales définies, edited by D. B. De Haan (P. Engels, Leide, 1867); note that there is a misprint in Eq. 4.521 .8 in Table of Integrals, Series, and Products, Sixth edition, edited by I. S. Gradshteyn and I. M. Ryzhik (Academic, London, 2000). The correct expression is $\int_{0}^{1} \mathrm{~d} x x(\arccos (x)) /\left(1+q x^{2}\right)^{2}=$ $\pi(\sqrt{1+q}-1) /(4 q \sqrt{1+q})$ for $q>-1$.

${ }^{64}$ See Eq. (26) on p. 136 in Tables of Integrals Transforms, Vol. I, Bateman Manuscript Project, edited by H. Erdelyi (McGraw-Hill, New York, 1954). 